\title{
Safety Review of Conceptual Fusion Power Plants
}

\author{
R. G. Clark
}

November 1976

Prepared for the Energy Research and Development Administration under Contract E(45-1)-1830

\section{Battelle}




\title{
NOTICE
}

This report was prepared as an account of work sponsored by the United States Government. Neither the United States nor the Energy Research and Development Administration, nor any of their employees, nor any of their contractors, subcontractors, or their employees, makes any warranty, express or implied, or assumes any legal liability or lespunsibility for the accuracy, completeness or usefulness of any information, apparatus, product or process disclosed, or represents that its use would not iniringe privately owned rights.

\author{
PACIFIC NORTHWEST LABORATORY \\ operated by \\ BATTELLE \\ for the \\ ENERGY RESEARCH AND DEVELOPMENT ADMINISTRATION \\ Under Contract EY-76-C-06-1830
}
Printed in the United States of America
Available from
National Technical Information Service
U.S. Department of Commerce
5285 Port Royal Road
Springtelc, Virginia 22151

Price: Printed Cosy 5

$\therefore$ Microfiche 53.00

$\begin{array}{lc}\text {-Pages } & \text { NTIS } \\ 001-025 & \text { Selling Price } \\ 026-050 & 54.50 \\ 051-075 & \$ 5.00 \\ 076-100 & 55.50 \\ 197-125 & \$ 6.00 \\ 126-150 & \$ 6.50 \\ 157.175 & 57.00 \\ 176-200 & 57.75 \\ 201-2.25 & 58.50 \\ 225-250 & 58.75 \\ 251.275 & 59.00 \\ 276-300 & 510.00 \\ & 510.25\end{array}$


BNWL-2024

UC-20

SAFETY REVIEN OF CONCEPTUAL FUSION POWER PLANTS

by

R. G. Clark

November 1976

Battelle

Pacific Northwest Laboratories

Richland, Washington 99352 
Fusion reactor technology has developed far enough to expect laboratory demonstration of practical levels of fusion employing the D-T reaction to occur in the early 1980s. Following that demonstration, and depending upon the national priorities for energy from D-T fusion, construction and operation of experimental reactors and demonstration power reactors could occur before the end of this century. Operation of the first commercial power plants could then follow, starting about 2010 .

Development and adoption of a new power system eventually will require a description of the environmental effects in an environmental statement providing a comparison to the effects of competitive systems. In anticipation of that statement, an environmental analysis (BNWL-2010) has been prepared for the ERDA Division of Magnetic Fusion Energy. That analysis estimates the environmental effects of constructing and operating D-T fusion reactors as an economically competitive source of electricity in the 21 st century.

The analys is has four primary purposes:

1. To describe the general nature of the environmental effects,

2. To determine current ability to estimate the effects,

3. To determine methods for reducing the effects, and

4. To determine research necessary for increasing capability to define and reduce the effects.

Timely identification of needed research and methods for reducing effects will permit the performance of that research and the revision of conceptual fusion power plant designs before preparation of the program environmental statement. This would improve the quality of the environmental statements and could reduce the estimated adverse environmental effects due to fusion power plants.

The environmental analysis (BNWL-2010) concludes that the following assumed characteristics are the best set for the first operating fusion power plants:
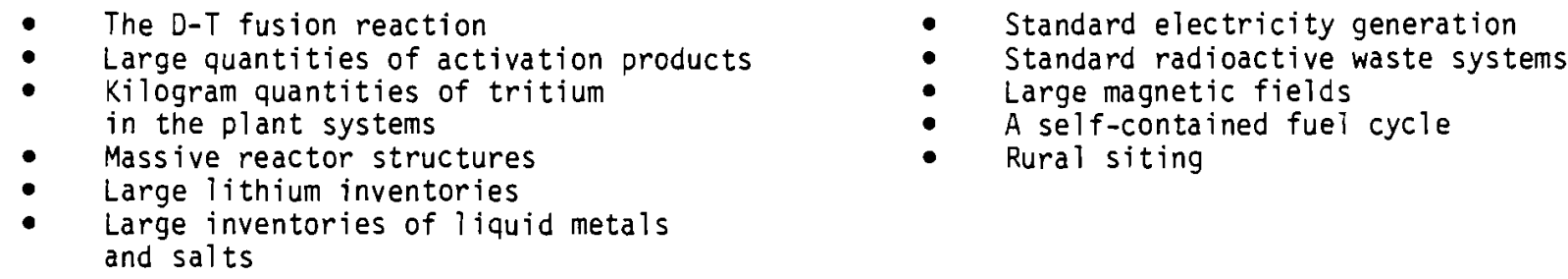

Using these characteristics a reference reactor was analyzed to determine the environmental effects by using available concepts of plant subsystems designs that control interactions with the environment or by assumption that best current technology would be used in subsystems design.

Because this analysis does not take into account advances in both fusion and waste control technology during the next thirty years, the estimated effects probabiy are significantiy higher than the actual effects will be for the first fusion power plants. The estimated environmental effects should be interpreted only as being the probable upper limit for the actual effects. 
Preparation of the fusion power plant environmental analysis required development and use of specially developed data and analysis methods not used in the preparation of current environmental statements for fossil and fission power plants. These data and analysis requirements are documented in a series of reference topical reports to make this information publicly available and to assure understanding of the basis for the conclusions made in the environmental analysis.

These reference topical reports sumarize the state-of-the-art as applicable to preparation of environmental statements for fusion power plants. They present the data and analytical techniques used in the environmental analysis to estimate the interactions with the environment and the resultant environmental effects. This information then was analyzed for adequacy and the need was determined for additional research to assure satisfactory ability to prepare environmental statements for the fusion development program and experimental facilities in the early 1980s. Estimated environmental effects are presented in these reference documents only as necessary to illustrate use of the data and analytical techniques.

This report is one of those reference documents for the environmental analysis. The other documents in this series contain more details of the power plant concepts and the probable environmental effects of fusion power plants with the assumed characteristics listed above. These documents are available through the National Technical Information Service:

An Environmental Analysis of Fusion Power to Determine Related R\&O Needs, BNWL-2010

Review of Fusion Research Program: Historical Summary and Program Projections, BNWL-2017

Fuel Procurement for First Generation Fusion Power Plants, BNWL-2012

Current Fusion Power Plant Design Concepts, BNWL-2013

Reference Commerical Fusion Power Plants, BNWL-2014

Siting Commercial Fusion Power Plants, BNWL-2015

Materials Availability for Fusion Power Plant Construction, BNWL-2016

Projected Themodynamic Efficiencies of Fusion Power Plants, BNWL-2017

Tritium Source Terms for Fusion Power Plants, BNWL-2018

Management of Nontritium Radioactive Wastes from Fusion Power Plants, BNWL-2019

Methodology for Estimating Radiation Doses Due to Tritium and Radiocarbon Releases, ENWL-2020

Magnetic Field Considerations in Fusion Power Plant Environs, BNWL-2021

Biological Effects of Tritium Releases from Fusion Power Plants, BNWL-2022

Biological Effects of Activation Products and Other Chemicals Released from Fusion Power Plants, BNWL-2023

Safety Review of Conceptual rusion Power Plants, BNWL-2024

An investigation of the Transportation Requirements of Fusion Power Plants. BNWL-2025

Considerations of the Social Impact of Fusion Power, BNWL-2026

Environmental Impacts of Nonfusion Power Systems, BNWL-2027

Environmental Cost/Benefit Analysis for Fusion Power Plants, BNWL-2028

Biomagnetic Effects: A Consideration in Fusion Reactor Jevelopment, BNWL-1973

An Analysis of Tritium Releases to the Atmosphere by a CTR, BNWL-1938 
The potential public safety impacts from accidents in conceptual fusion power plants were investigated. Fusion was found to have some potential for accidents, as does any energy generating system. Functions of fusion power plants were identified that possess sufficient potential for an accidental release of toxic materials to the environment. An assessment was made of the impact of the potential accidents and recommendations are included for R\&D that will allow incorporation of safety concerns in fusion power plant design. This work was based on a review of information available in conceptual design documents of fusion reactor systems.

PNL recommends that a continuing effort be made to develop priorities for fulfilling safetyrelated research needs consistent with the significance of the safety item, the uncertainty of the estimated environmental effects (based on existing technology), the time required to develoo the information, and the date when this information is needed. Guidance is proposed as to how this activity should be conducted.

Inventories of potentially dispersible toxic materials identified in current fusion reactor concepts were categorized into four general classifications: tritium, corrosion products, air activation products, and nonradioactive materials. Identification of possible release mechanisms of these toxic materials and their dispersibility was not attempted. However, some general conclusions were made about their relative importance.

- Tritium is a biologically significant, potentially dispersible material present in significant quantities in fusion power plants. The projected inventories of tritium will greatly exceed inventories presently being managed in the nuclear industry. Even though considerable information and experience is available on handing and containing tritium, significant design and development work will be required to extend the technological base to safely manage quantities currently anticipated for fusion reactors.

- Radioactive species added to the coolant of a fusion power plant either by sputtering or corrosion provide a potentially dispersible source of radioactive material which must be considered in future designs.

- Air activation is possible due to the high neutron radiation fields present in fusion reactors. Activated air represents a small potential source of dispersible radioactive material. Design considerations to satisfactorily manage this seem to be straightforward.

- Large inventories of toxic nonradioactive materials appear in some fusion reactor designs. Conceptual designs for fusion reactors are incomplete and no specific conclusions could be reached about the environmental impact from these materials but they must be considered in design. 
Stored energy sources that could cause the initiation or propagation of an accident in a fusion reactor were identified for the various conceptual plant types. The potential sources were surmarized in five categories: plasma energy, magnet energy, thermal energy, chemical energy, and cryogenic energy.

The conclusions reached on the safety of the stored energy are based on the assumption that the releases of these energies occur as single events. Scenarios where the release of these stored energies may initiate or propagate the release of other energies were not specifically considered. However, during detailed design, combination of these potential energies must be considered to insure that such scenarios will not present a hazard to the public or the environment. General conclusions reached about the safety implications of these energy sources are:

- The available energy of fusion from the approximately one gram of deuterium-tritium confined in a plasma will not be a safety concern.

- The kinetic energy of the plasma has the potential to breach the vacuum wall if it should dissipate in localized areas due to instabilities and power surges of the plasma. As a single event, the safety of the public is not involved, al though significant plant damage may result.

- A large amount of stored energy exists in the magnets considered for fusion reactors. Magnet failures have the potential to cause extensive damage to the magnets themselves, to vaporize material and to release small amounts of contamination into the containment structure. Even though the stored energies are very large, magnet failures do not in themselves appear to be capable of penetrating a well-designed containment structure. However, design effort will be required to mitigate any rapid energy release from magnets.

- A temperature rise will result from blanket afterheat if coolant is lost in a fusion reactor, but the rate of temperature rise is not sufficient to require immediate emergency cooling. Perspective on this can be obtained by noting that fission reactor decay heat is about 7\% of operating power level, whereas fusion reactor decay heat is $1 \%$ or less of reactor power.

- The chemical energy in liquid metal coolants specified in several designs provides potential for releasing radioactive material and causing structural damage in the plant. Therefore,liquid metal fires and reactions of liquid metals with concrete and water are primary safety concerns for fusion reactors. The research programs for cleveloping LMFBR technology are expected to make majer contributions to the technology for safe operation of liquid metal cooling systems, including those proposed for fusion reactors.

- Cryogenic energy release resulting in significant helium volume changes and in rapid thermal cycling could caise structural damage in the reactor. However, the available stored energy is not uniquely high. The necessary design considerations to handle these situations will likely require additional development as the concerns are identified. 
Some insight into the safety significance of various toxic materials contained in conceptual fusion power plants was developed by determining the consequences of releasing unit quantities of potential pollutants. A measure of environmental significance is the public dose received from the release of a toxic material. This dose is a function of many parameters, including quantity, physical form and type of material released, duration of release, atmospheric conditions, and distance. Dose consequence calculations were made for the more relevant radioactive materials expected to be contained in fusion power plants on a unit source basis. Such dose information can be evaluated to determine which isotopes and materials are significant and should receive design emphasis.

An assessment method was developed and proposed as a means to identify potential accident scenarios as designs become available. The method provides a means for analysis of the system interactions and barriers that must be defeated by an accident in a reactor system in order to represent a potential public safety concern. Plant malfunction and accident initiating events were identified that potentially could lead to release of contaminants to the environment. The assessment of these events is general because of the limited desian information.

The same logic was also used to identify some technological areas where an investment in research and development would lead to a better understanding of the safety implications of fusion facilities and ennance the technical bases for design. Research and development recormendations are provided for both the safety to the public, and operating and maintenance personnel within the facility itself. Specific areas identified for research and development relating to public safety are:

- Primary boundary failure

- Abnormal plasma transients

- Cryogenic system malfunctions

- Containment analysis

- Abnormal primary flow transients

- Magnet structural analyses.

Specific recommendations for research and development related to operational safety are made for the following areas:

- Primary boundary leakage

- Instrumentation evaluation

- Operational and remote maintenance analysis

- Radioactive material inventory and location. 


\section{CONTENTS}

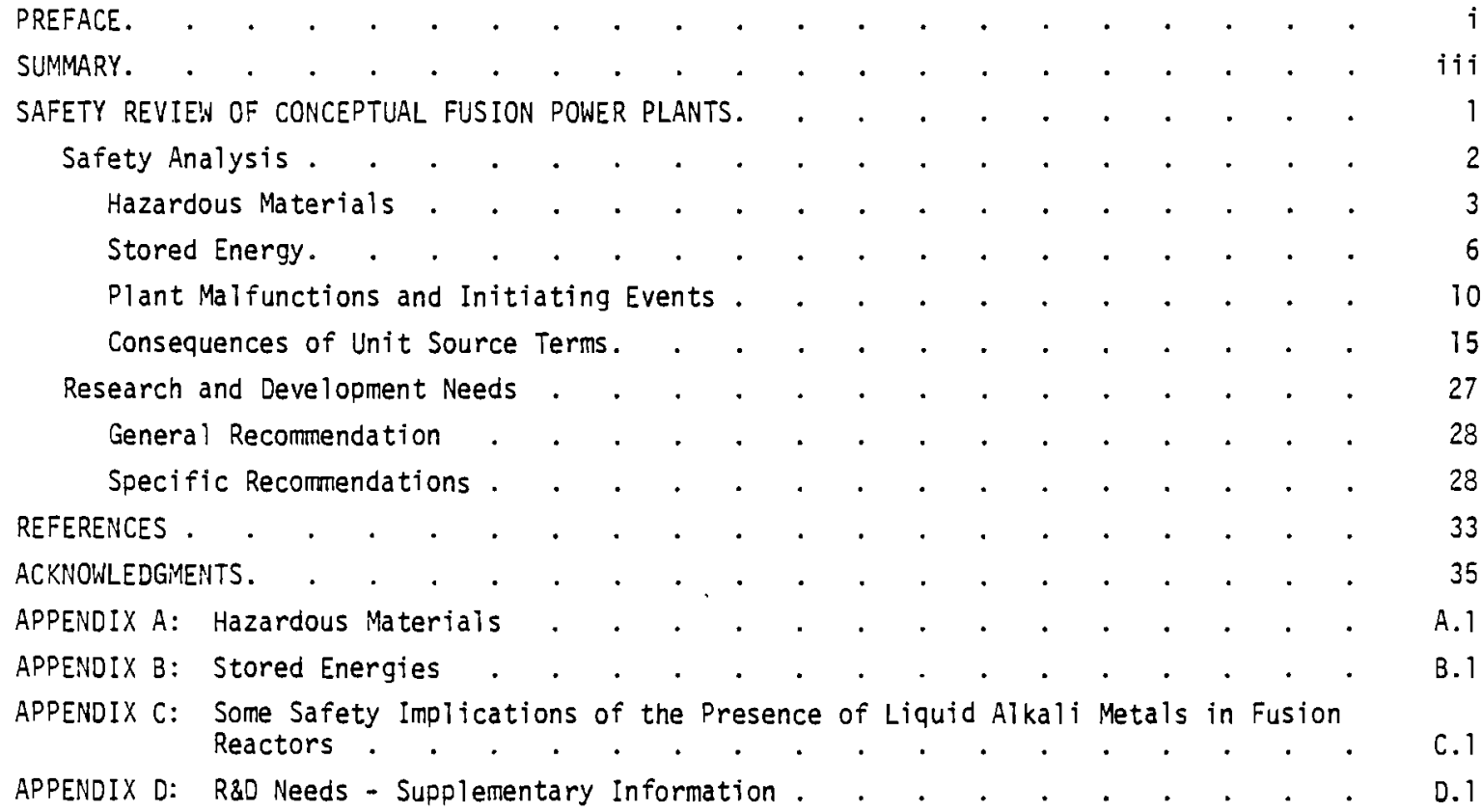




\section{LIST OF FIGURES}

1 Afterheat Versus Shutdown Time for Niobium and Vanadium and for Fission Reactor Fuels

2 Safety Analysis Logic for Magnetic Confinement Fusion Reactors

3 Total Body Dose as a Function of Distance Following the Release Over an 8-Hour Period of $1000 \mathrm{Ci}$ of Tritium as an Oxide

4 Inhalation Dose Versus Downwind Distance for a $1 \mathrm{Ci}$ Release at Ground Level of $1\lrcorner$ sized Particles of $95 \mathrm{Nb}$ and $55 \mathrm{Fe}$ in Soluble Form

5 Inhalation Dose Versus Downwind Distance for a $1 \mathrm{Cj}$ Release at Ground Level of 1 is Sized Particles of $95 \mathrm{Nb}$ and $55 \mathrm{Fe}$ in Insoluble Form

6 Inhalation Dose Versus Downwind Distance for a $1 \mathrm{Ci}$ Release from a Height of $100 \mathrm{~m}$ of 1 \& Sized Particles of $95 \mathrm{Nb}$ and $55 \mathrm{Fe}$ in Soluble Form

7 Inhalation Dose Versus Downwind Distance for a $1 \mathrm{Ci}$ Release from a Height of $100 \mathrm{~m}$ of 1 is Sized Particles of $95 \mathrm{Nb}$ and $55 \mathrm{Fe}$ in Insoluble Form

J-1 Safety Anaiysis Logic for Magnetic Confinement Fusion Reactors

0.1 


\section{LIST OF TABLES}

1 Conceptual Power Plant Designs

2 Range of Tritium Inventories in Various Systems in the Conceptual Magnetic 4 Confinement Fusion Reactors

3 Estimated Annual Inventory of Corrosion Products in UWMAK-I Systems 5

4 Gaseous Activation Products Produced from Air in UWMAK-I 5

5 Estimated Stored Energies in Magnets for Conceptual Fusion Reactors 7

6 Stored Thermal Energy in Conceptual Fusion Reactors Primary Coolant Systems 10

7 Potential Plant Malfunctions for Conceptual Fusion Reactors 11

8 Potential Initiating Mechanisms for Accidents for Conceptual Fusion Reactor 13

9 Physical, Biological and Effective Half Lives in the Human Body of Isotopes of 17 Interest in Fusion Power Plant Safety Studies

10 Unit Source Term - Consequence Calculations Input Parameters 18

11 Dose as a Function of Downwind Distance Following the Release of Various 19 Radioactive Substances which may be Present in CTR's

12 Relative Concentration of Radioisotopes in Conceptual Fusion Reactor Blankets 24

13 Recommendations for Safety Research and Development Relative to Accidents 29

14 Recommendations for Research and Development for Operational Safety 31

A-1 Compilation of Potentially Hazardous Materials A.2

B-1 Stored Energy in Conceptual Fusion Reactor Systems B.2

C-1 Alkali Metal Reactions C.2

C-2 Composition of Conventional Concrete (Type $\mathrm{Cl}-\mathrm{P}$ ) and Reaction Products $\quad$ C.4

C-3 Composition of Magnetite Concrete (Type M-225) and Reaction Products 
This document discusses potential public safety impacts that could result from accidents at fusion power plant facilities. Detailed evaluation of the potential impact of operating these future facilities is not possible due to the conceptual nature of fusion reactor designs. The approach taken in this study was to assemble and review the information available on the safety considerations of fusion systems. Analyses in the literature for individual concepts (Table 1) form the bases for the conclusions of this report and are referenced throughout. The technical information supports the conclusions about the safety aspects of fusion power contained in BNWL-2010, "An Environmental Analysis of Fusion Power to Determine Related R\&D Needs."

TABLE 1. Conceptual Power Plant Designs

Magnetic Confinement

Tokamak

University of Wisconsin (UWMAK)

Brookhaven National Laboratory (BNL)

Princeton Plasma Physics Laboratory (PPPL)

Dak Ridge National Laboratory (ORNL)

Theta Pinch

LOS Alamos Scientific Laboratory (LASL)

- Mirror

Lawrence Livermore Laboratory (LLL)

An analytical approach similar to a Preliminary Hazards Analysis (PHA) was used to organize the information from fusion power plants that could affect public safety. Design characteristics of conceptual fusion systems were used to develop three categories of safety-related information:

1. Hazardous Materials. The identity and characteristics of hazardous substances.

2. Stored Energy. That which could provide the driving force for an uncontrolled release.

3. Plant Malfunctions. Occurrences that could be the initiating event leading to an uncontrolled release of toxic material.

The environmental consequences of unit source terms were calculated to provide a measure of the relative significance of some of the toxic materials contained in fusion systems. These consequence evaluations, given in the form of radiation dose versus distance curves, can assist the fusion safety analyst and system designer when considering potential accidental emissions.

It is assumed that operating fusion reactors will provide adequate consideration of safety to the public. Thus, this report primarily attempts to highlight those areas where there may be safety concern which should be considered in future fusion designs. It is also recognized that future safety analyses will need to be quantitative. Therefore, recommendations are made for research and development efforts to better define or manage safety concerns. 


\section{SAFETY ANALYSIS}

Present fusion power plant concepts contain materials that if accidentally released from the facility could be a public safety concern. Large amounts of energy are expected to be confined within fusion reactor systems. Malfunction of any one system, or of several systems concurrently, could produce driving forces of sufficient strength to release quantities of these toxic materials. Design of fusion power plants must provide containment for these materials under the forces that could be imposed by nomal operating and potential accident conditions. This analysis defines the hazardous materials, stored energies, and potential plant malfunctions that could lead to releases of material.

The information needed for a quantitative safety assessment include:

- Detailed system design information.

- Quantities and characteristics of toxic material on the reactor system(s). Behavior of toxic materials under accident conditions and in the environment.

- Potential energy held in the reactor system(s) that could initiate or propagate an accident.

- System performance under normal and accident conditions.

- Initiating events that can lead to plant malfunctions and releases of toxic material to the environs.

Using this information, the effects of accidents are estimated by postulating and evaluating potential scenarios that could lead to the uncontrolled release of toxic materials from the plant. Adequate understanding of the impact from accidents at fusion power plants can be achieved if all of the important scenarios can be identified and properly evaluated in terms of effects on the public and environment.

Experimental data and analytical methods are also needed to support the evaluation of the release scenarios. Such information is used to predict system behavior and interaction of systems under accident conditions. The movement of toxic material through confinement/containment barriers to the environment are modeled using similar information. Once the toxic material is released from the facility, analytical models and validation data are required to quantitatively estimate the consequences.

Special analysis techniques can be used to aid in understanding the safety of the reactor systems consistent with the degree of design detail and system performance information available. These methods range from preliminary hazards analysis (PHA) where minimum information is available to comprehensive risk analysis when detailed design, operating and system performance information is available.

At this time, only conceptual fusion design information is available; thus, the safety analysis could be performed only to the depth of a PHA. This type of analysis then formed the basis for the conclusions on fusion reactor safety made in this report. 
Hazardous Materials

The potentially dispersible radioactive and chemically toxic materials known or implied to be present in conceptual fusion reactors with magnetic confinement include materials located in the blanket, the heat transfer system, the spent plasma collection, and the bred tritium recovery system. The inventories of these materials are compiled in Appendix $A$. The information comes from design documents ${ }^{(1-8)}$ and fusion reactor safety assessments. ${ }^{(9-15)}$ corrosion and activation products data are extracted from a topical report on radioactive waste from fusion power plants. (16)

The compilation was made to identify the materials which could be important for future design consideration because of safety impications. Chemically toxic substances are listed if they are highly reactive or if an Occupational Threshold Limit Value (TLV) has been set by the American Conference of Governmental Hygienists. Other materials for which no TLV exists but which may prove hazardous are also listed. Possible release mechanisms or dispersibility of the particular material was not considered.

Examination of the inventories and potential effects of the materials reveals four general classifications of toxic materials that could be of concern in accidents at fusion power plants. These are tritium, corrosion products, air activation products, and nonradioactive materials.

1. Tritium

Tritium $\left({ }^{3} H\right)$ is the radioactive isotope of hydrogen. It decays with a 12.3 year half life and emits a low energy beta particle $(18 \mathrm{keV})$. In the oxide form, tritiated water $\left(T_{2} 0\right)$, acts chemically the same as normal water and assimulates with normal body fluids when taken into the body. The tritium uniformly deposits its energy to the body during deçay until it is eliminated with the normal body water half-life of about 10 days. However, a small fraction $(<1 \%)$ can become organically bound with a biological half-life of about 450 days. (17) Because of this longer half-life, the small percentage of bound tritium is estimated to contribute about $16 \%$ of the total dose. (17) Earlier work reported higher estimates of bound tritiated body water with even a longer biological half-life. (18)

A release of gaseous tritium is of lesser immediate biological consequence than release of the oxide. (19) It has been estimated $(20,21)$ that only about $0.2 \%$ of tritium gas released during an accident will be immediately converted to tritiated water vapor. Eventually, all of the tritium is converted to the oxide in the environment. (22) Prior to the conversion process, however, the tritium gas will be dispersed, reducing the potential exposure to local populations.

Current fusion reactor concepts contain total plant inventories up to 24 kgs of tritium. (23) The majority of this tritium is either as a metallic compound (e.g., LiT) or as a gas (e.g., T2, $T_{2} \mathrm{O}$ ). Since tritium is present in virtually every system of conceptual fusion power plant designs (Table 2), the potential for a tritium release exists in nearly every plant accident.

Tritium is a unique concern of fusion reactors as they are presently conceived. The projected inventories of tritium will greatly exceed inventories previously managed at one location. 
TABLE 2. Range of Tritium Inventories in Various Systems in the Conceptual Magnetic Confinement Fusion Reactors

\begin{tabular}{lc}
\multicolumn{1}{c}{ System } & Inventory \\
Blanket & $10-400 \mathrm{~g}$ \\
Blanket Coolant & $1-8.7 \mathrm{~kg}$ \\
Steam* & $<7 \mathrm{~g}$ \\
Spent Plasma Collection & $0.5-3.5 \mathrm{~kg}$ \\
Cryopumps** & $0.3 \mathrm{~kg}$ \\
Bred Tritium Recovery & $0.4-1.0 \mathrm{~kg}$ \\
Storage & $1.4-10 \mathrm{~kg}$ \\
Total Plant Inventory & $3-24 \mathrm{~kg}$
\end{tabular}

* Only value given is for the theta pinch

** Only value given is for UWMAK-I

Acknowledging that releases of tritium as an oxide will have the greatest potential environmental impact, design efforts could be made to preclude or minimize accident scenarios leading to the release of tritium as an oxide. Some perspective on the potential for the overall impact of fusion power on tritium in the environment is given in :ASil-1250.* Even though considerable information and experience is available on handling and containing tritium, significant design and development work will be required to scale up the systems to safely manage the quantities currently anticipated for fusion reactors.

\section{Corrosion Products}

First wall and blanket structure activation products will be major contributors to the radioactive inventory at a fusion power plant. When these materials are bound up in structural materials, they are of significance only in an accident that could vaporize portions of these structures. However, small quantities of these materiais are continuously removed from the structures by sputtering of the first wall and corrosion of piping by liquid metal coolants. The tabulation of corrosion products postulated for U!lifk-1 are given in Table 3 . This was taken from Appendix $A$. These corrosion products are essentially all metallic oxides with oxides of iron the principal contributor followed by those of nickel and thorium.

Scavenging techniques will remove most of these contaminants from the process systems and concentrate them in collectors. They then have some potential for being dispersed either by fires or other disruptive accidents. Fusion power plant designs will require measures to insure that these concentrated inventories of corrosion and sputtering products cannot be released in significant quantities during an accident.

* USAEC, The Safety of Nuclear Power Reactors and Related Facilities, WASH-1250, p. 4-19, Figure 4-6, July 1973. 
TABLE 3. Estimated Annual Inventory of Corrosion Products in UWMAK-I Systems $(6)$

Annual Production of Corrosion Products

Amount Type Radioactivity, $\mathrm{Ci}$

$\begin{array}{lccc}\text { Blanket } & -2.5 \mathrm{MT} & \text { Metal Oxides } & 4 \times 10^{6} \\ \text { Heat Transfer System } & 269 \mathrm{~g} & \text { Metal 0xides } & 400 \\ \text { Spent Plasma } & 1.6 \mathrm{MT} & \text { Metal 0xides } & 2.4 \times 10^{6} \\ \begin{array}{l}\text { Bred Tritium } \\ \text { Recovery System }\end{array} & -100 \mathrm{~g} & \text { Metal Oxides } & 150\end{array}$

3. Air Activation Products

Air leaking into the vacuum of the plasma region of a fusion reactor will become activated in the high neutron fluxes present. Some perspective on the magnitude of the inventory of radioactivity from air activation is possible by reviewing an analysis (16) originally completed to show the need for an evacuated region to surround the plasma confinement changer.

This analysis predicts the daily radioactivity production in the plasma confinement chamber from in-leakage of air from the secondary confinement volume. The assumption is made that the secondary confinement volume contains air at one atmosphere and that this in-leakage continues for one day with the reactor operating. Normally, the secondary containment system that surrounds the plasma confinement chamber is held at a low partial pressure. It is expected that the reactor would not operate at all under such conditions. Hence, the hypothetical activation levels estimated here can be assumed to represent maximum values. The results of the analysis are summarized in Table 4. The levels are nominal.

TABLE 4. Gaseous Activation Products Produced from Air in UWMAK-I (16)

\begin{tabular}{|c|c|c|c|c|}
\hline $\begin{array}{l}\text { Product } \\
\text { Nuclide } \\
\end{array}$ & $\begin{array}{l}\text { Production Rate } \\
\text { Curies/day } \\
\end{array}$ & $\begin{array}{l}\text { Activity at End } \\
\text { of } 1 \text { Day, Curies } \\
\end{array}$ & $\begin{array}{l}\text { Activity After } 2 \mathrm{Hr} \\
\text { Hold-Up, Curies } \\
\end{array}$ & $\begin{array}{l}\text { Hold-Up Time to } \\
\text { Reduce to } 1 \mathrm{mCi}\end{array}$ \\
\hline$N-13$ & $4 \times 10^{6}$ & $4 \times 10^{4}$ & 9.4 & $250 \mathrm{~min}$ \\
\hline$N-16$ & $3 \times 10^{3}$ & $3 \times 10^{-2}$ & 0 & $36 \mathrm{sec}$ \\
\hline$C-14$ & $8 \times 10^{-2}$ & $8 \times 10^{-2}$ & 0.08 & $36,000 \mathrm{yr}$ \\
\hline$A r-4 T$ & $2 \times 10^{2}$ & $2 \times 10^{1}$ & 11 & $27 \mathrm{hr}$ \\
\hline
\end{tabular}

The quantity of air activation products generated in the secondary containment volume during these same conditions would be significantly less because of the much lower neutron flux level (corders of magnitude). The short half lives of the radioactive nitrogen exclude them from contributing significantly. Any air activation products could disperse to the atmosphere almost immediately however if the containment vessels were breached. These must at least be reviewed when source terms for accident scenarios are developed. 


\section{Nonradioactive Materials}

The release mechanisms for many of these nonradioactive materials is not clear at this time. The actual importance of these items in assessing the impact from accidents at fusion power plants will require the development of accident scenarios and realistic source terms.

The large inventories of some of these materials, such as mercury, suggest that they be considered in system designs and future accident analyses.

\section{Stored Energy}

In this section, potential energy sources are identified that theoretically could initiate or propagate accidents by virtue of its release. The large stored energies for the conceptual fusion reactor designs are organized by plant type in Appendix B. In many cases, the designs lack sufficient detail to precisely calculate the stored energies in the reactor system. Therefore, many of the numerical values are estimates. All sources of stored energy are identified in five categories: plasma energy, magnet energy, thermal energy, chemical energy and cryogenic energy.

\section{Plasma Energy}

Plasma Fusion Eneray - The large confinement volume of the torus of a 5000 MWt fusion reactor is typically charged with about 1 gram of fuel material. (6) Normally, about $5 \%$ of this fuel burns in an operating cycle. To gain perspective on the levels of energy hypothetically available from the plasma, an analysis was made to estimate the energy. Although it is physically impossible, complete fusion of the 1 gram $(100 \%)$ of the fuel would liberate about $10^{12}$ joules of energy. (6) To continue the hypothesis, if this amount of energy were dumped uniformly to the first wall in a few seconds, the blanket temperature would increase by $50^{\circ}-100^{\circ} \mathrm{C}$ and the first wall temperature would raise to $600^{\circ} \mathrm{C}$. The primary boundary will not be breached $(10,24)$ from this temperature.

Plasma Kinetic Energy - Very high plasma temperatures are required to initiate and maintain the fusion reaction. As a consequence, the unburned plasma in the confinement chamber contains a significant amount of kinetic energy. It has been estimated $(25)$ that 500-4000 megajoules of kinetic energy are contained in the plasma of a 5000 MWt fusion reactor. Deposition of this energy uniformly over a $5-m m$ thick niobium first wall has been calculated to result in a temperature rise of $60^{\circ}-100^{\circ} \mathrm{C}$. (25) This would be of minor concern. A localized deposition of this amount of energy to the first wall however could result in vaporization of the wall material at that point and the possible release of blanket coolant into the confinement chamber. But, it is physicaliy impossible for such an event to occur with the large reaction plasmas conceived to date. This is because of the distribution of this plasma kinetic energy over a rather large volume and there is no known plasma event which could conceivably allow the local deposition of all this energy. There are possible events in which a significant amount of plasma energy can be imported to the vacuum chamber wall through a kink instability, causing a section of the plasma to touch the wall and short the plasma since presumably the wall is grounded. 
Even then, most of the energy is conducted away from the local spot and either sent to a ground or distributed throughout the wall. Such an accident could result in damage and a reactor shutdown. No environmental impact would be expected from this accident as a single event.

\section{Magnet Energy}

Magnets specified for magnetic confinement fusion reactors contain large stored energies (Table 5). The manitude of this stored energy source offers significant potential to damage equipment or be a motive force for the release of toxic material. The potential for damage of reactor components during such a release of energy is related to the rate of energy relezse. Magnetic fields have some inherent stability in the rate of releasing stored energy. However, the large stored energies in fusion magnets resulting from the very high currents in superconducting coils and the large cores will require designs to control the potential for unplanned, rapid energy release from the system.

TABLE 5. Estimated Stored Energies in Magnets for Conceptual Fusion Reactors(23)

\begin{tabular}{lr} 
Reactor Concept & $\begin{array}{r}\text { Stored E } \\
\text { MJ } \times 10\end{array}$ \\
\cline { 3 - 3 } UWMAK & 3.5 \\
Theta Pinch & -0.6 \\
PPL & 2.5 \\
ORNL & -1.0
\end{tabular}

The major concerns of magnet systems relative to public safety are:

- Joule heating within a magnet or conductor sufficient to vaporize material.

- Sudden helium vaporization from heating resulting in destructive rupture of the helium coolant system.

- Thermal stress ruptures of magnets.

- Electric arcing with material vaporization and generation of high temperature flying material.

- Generation of eddy currents and stray electric fields.

A possible magnet failure mode is loss of superconductivity in one or more coils. A loss of superconductivity, causing a coil or coils to revert to normal conductivity, does not of itself result in significant effects. Since the electrical current is inductively coupled to other coils, part of the energy is transferred through their mutual magnetic fields. The remainder of the energy dissipates as heat in the nonsuperconducting coil material. The energy dissipation may heat the coil only a few hundred degrees, at most, assuming a uniform distribution throughout the conductor. However, localized dissipation could have serious consequences to the magnets, including tisermal ruptures. 
Rapid field distortion due to loss of a coil would also induce large electrical potentials. Normally, compensating changes in other superconductor coil currents would limit the growth of stray electric fields. However, since the superconductors are expected to operate near their saturation field cutoff for cost effectiveness, the additional current load in adjacent coils could conceivably exceed saturation and revert them to normal conduction as we1l. The resulting cascade would generate a very high local electrical field that would manifest itself in arc discharges and large electromagnetic wave amplitudes. The possibility of material vaporization and dislocation exists. Magnet designs will probably require some energy dumping device to cope with this problem.

The joule heating caused by a transition from its superconducting to the normal state can result in the vaporization of the liquid helium used to cool the magnets. This could generate large pressures in the cryogenic system. These problems are discussed in the section on cryogenic Energies.

Magnetic field transients in the superconducting coils can produce large eddy currents in nearby conductors. The forces produced in the conductor by these eddy currents can be quite large. Material dislocation could result unless conductors in the vicinity of the magnets are designed to withstand these large forces.

Magnet faitures have the potential to cause extensive damage to the magnets themselves, to vaporize material and to release small amounts of contamination into the containment structure. Even though the stored energies are very large, magnet failures do not in themselves appear to be capable of penetrating a well-designed containment structure. Design effort will be required, however, to control the potential for inplant damage from magnetic failures to acceptable leveis.

\section{Thermal Energy}

81 anket afterheat, from radioactive decay of activated blanket materials, is the source of most residual heat in a fusion reactor following a plasma shutdown. If a loss of coolant accident occurs, the temperature rise of the niobium blanket structures has been estimated to be about 0.13 to $0.18^{\circ} \mathrm{K} / \mathrm{sec}$ with a maximum temperature of $1200^{\circ} \mathrm{K}$ developed. $(14,24)$ This temperature has the potential to produce internal plant damage. From a safety standpoint, the potential for release of radioactive contaminants is sufficiently remote from this accident that emergency cooling for public safety is not required. The decay heat energy from most fusion blanket designs can be removed to a large extent by conduction and radiation. (12)

The specific amount of decay heat is highly dependent on the design of the blanket. Predictions ( of the afterheat as a function of time after shutdown are presented in Figure 1 for structural materials niobium and vanadium (which has been proposed as a substitute for Nb). Stainless steel afterheat is expected to fall between these two curves. Other blanket materials that have been proposed such as sintered aluminum (SAP), carbon and silicon carbide would significantly reduce blanket activation and the amount of energy stored in the blanket. The decay heat curves for these materials would fall below those for vanadium. 
For perspective, an estimate of the decay heat from a fission reactor is shown in Figure 1 . Note that at time of shutdown the fission reactor decay heat is about $7 \%$ of the operating power level, while in a fusion reactor it is $1 \%$ or less of reactor power. Rapidly responding emergency cooling systems to protect the public from the consequences of a loss of cooling accident do not appear to be required in fusion reactors, based on conceptual designs.

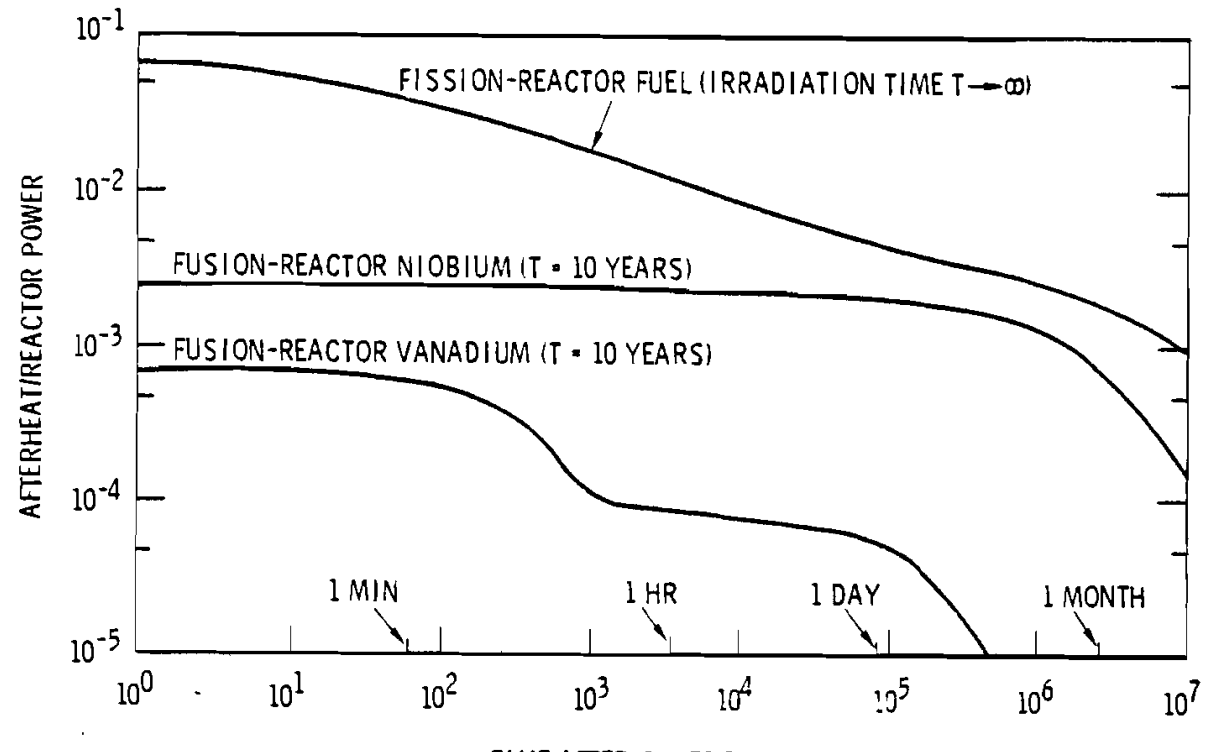

TIME AFTER SHUTOOWN, SEC

FIGURE 1. Afterheat Versus Shutdown Time for Niobium and Vanadium and for Fission Reactor Fuels (15)

Another source of thermal energy stored in fusion reactors is in the coolant system. The energy stored in the primary coolant systems for the conceptual fusion reactors is shown in Table 6 . Coolant temperatures are high relative to light water reactors but are consistent with the technology for liquid metal and helium coolant systems. Thermal energies of this magnitude in the coolant systems are managed effectively with current technology. Management of this energy under accident conditions will not represent a design problem unique to fusion reactions.

\section{Chemical Energy}

Liquid alkali metal coolants are specified in several of the fusion reactor designs. The chemical energy in these liquid metals coupled with the thermal energies of operating conditions 
TABLE 6. Stored Therma 1 Energy in Conceptyal Fusion Reactors Primary Coolant Systems $(22)$

\begin{tabular}{|c|c|c|c|c|c|}
\hline Concept & Coolant & $\begin{array}{c}\text { Exit } \\
\text { Temperature } \\
\left({ }^{\circ} \mathrm{C}\right) \\
\end{array}$ & $\begin{array}{r}\text { Stored } \\
\text { Joul es } \times 10^{1}\end{array}$ & $\begin{array}{l}\text { rgy } \\
\text { GW-Sec }\end{array}$ & $\begin{array}{l}\text { Reactor } \\
\text { Power } \\
\text { (GWe) } \\
\end{array}$ \\
\hline UWMAK-I - & Li & 489 & 9.2 & 920 & 1.5 \\
\hline BNL & $\mathrm{He}$ & 760 & 0.15 & 15 & 1.6 \\
\hline PPL & $\mathrm{He}$ & 638 & 0.64 & 64 & 2.0 \\
\hline ORNL & Li & 1052 & 3.3 & 330 & 0.5 \\
\hline Theta Pinch & Li & 547 & 16.3 & 1630 & 4.1 \\
\hline
\end{tabular}

are a significant source of stored energy. At the coolant temperatures proposed, the liquid metals would ignite spontaneously in air (if present) and would also react vigorously with water or concrete. Significant amounts of thermal energy would be involved, with the possible volatilization of radioactive blanket materials or corrosion products. Tritium entrained in some of the coolants could be released and hydrogen gas generated. A detailed discussion of some of the safety implications, i.e., combustion mechanisms, associated with liquid metals is presented in Appendix $C$. Note that significant amounts of radioactive materials may be released and the potential for structural damage exists. For these reasons, liquid metal fires and the reactions of liquid metals with concrete and water appear to be primary safety concerns in fusion reactors.

For a liquid metal fire to develop in the vacuum region of the reactor, penetration of the containment is required. Reactions with concrete can result only after penetrating stainless steel liners covering the concrete. Designing, constructing and operating liquid metal cooled fission reactors (LMFBRS) has contributed and will continue to contribute technology for safe operation of liquid metal cooling systems including those proposed for fusion reactors.

\section{Cryogenic Energy}

Cryogenic systems using the liquid helium coolants are required in several of the systems proposed for fusion reactors. As indicated in Appendix $B$, Note $C$, helium changes volume by a factor of 718 in going from a liquid at $4^{\circ} \mathrm{K}$ to a gas at room temperature. The pressures associated with such a volume change in a confined area could be very large. The concurrent failure of pressure control or pressure relief devices could result in loss of structural integrity. Confinement could be affected. The designs necessary to eliminate any realistic probability of this occurring are straightforward. A minimum effort should be required to preclude such a potential event from occurring, especially since the confinement volumes are expected to be very large.

Plant Malfunctions and Initiating Events

With the inventories of hazardous materials and the potential forces (stored energies) expected to be held in fusion systems, it is important early in design to identify unique events for 
fusion reactors that, if they were to occur, could potentially lead to public safety concerns. Such events have been called Plant Malfunctions.

A preliminary listing of such events is given in Table 7 . The 1 isting includes events from all magnetic confinement fusion reactor concepts. For use in design, the 1 isting would be 1 imited to a specific reactor concept. The tabulation was developed from comparisons of similar analyses for fission reactor systems, consideration of stored energies, hazardous material inventories, and prior engineering analyses. $(6,9,26)$

TABLE 7. Potential Plant Malfunctions for Conceptual Fusion Reactors

I. Magnet System Malfunction

1. Magnet Failure

II. Cryogenic Sys tem Malfunction

1. Failure of Cryogenic System

III. Liquid Metal Reactions

1. a. Lithium Fire

b. Lithium Exposure to Concrete

2. a. Sodium Fire

b. Sodium Exposure to Concrete

3. a. Potassium Fire

b. Potassium Exposure to Concrete

IV. Containment Structural Failure Malfunction

1. Containment Penetration

a. Internally Generated Missiles

b. Externally Generated Missiles

2. Containment Overpressurization

V. Hazardous :Material Release

1. Tritium Release from Storage

2. Tritium Release from Waste Handling Activities

VI. Primary Boundary Failures

1. Severe Overpower Transients

2. First Wall Failure

3. Failure of Spent Fuel Recovery System

4. Loss of Primary Cooling

5. Loss of Secondary Cooling 
Additionally important for design consideration are the causal events, namely, the events that can initiate or propagate accident sequences leading to a plant malfunction. Such events have been called Initiating Events. A listing of the initiating events that could be identified at this time is given in Table 8 . The initial events were identified considering specific plant malfunction and accident scenarios. Development of accident scenarios and initiating mechanisms necessarily require a thorough knowledge of reactor systems and their interaction as well as plant operating information. This being the case, continuing evaluations of potential accident initiating events should be made parallel to fusion reactor development to assure their proper consideration in design.

To aid in the identification of plant malfunctions and initiating events, a logic diagram shown in Figure 2 was developed for a magnetic confinement reactor. The diagram illustrates system interaction and the barriers that must be defeated for the accident to represent a potential environmental effect. Each box in the diagram represents analysis that must be made or data that must be available to determine if potential accidents can propagate. This diagram along with similar logic is used in the section on R\&D Needs, to identify the information and analysis methods that need to be developed for future designs.

Significant releases of toxic materials can only occur after failure of the reactor containment system. Such a failure could occur due to either internal plant events or external events. External events include earthquakes, tornadoes, floods, and missile impact (e.g., airplane, meteor). Such events are site specific and designs will need to incorporate appropriate safety features. Plant events that could conceivably cause or lead to the breach of plant containment have been discussed in the previous section. The more significant of these are liquid metal fires and/or reaction with concrete, magnetic failures, and cryogenic system failures. As single events, liquid metal reactions appear to pose the more significant threats to the containment integrity.

Although mentioned in the previous section, a more detailed discussion is presented in the following paragraphs for three potential plant malfunctions; namely, uncontrolled power excursion, plasma instabilities, and loss of cooling.

Uncontrolled Power Excursion. Most of the designs considered maintain plasma ignition by the injection of energetic fuel atoms. The plasma temperature is maintained to a large degree by the absorption by the feed material of a portion of the energy of alpha particles produced in the $D-T$ reaction. A loss of feed would reduce the plasma reaction rate. A decrease in the reaction rate will cause the plasma to cool and lose ignition. Loss of feed then appears to be incapable of producing a hazardous condition.

Various analyses $(12,13)$ have concluded that excess feed cannot lead to a runaway overpower transient. The increased plasma reaction rate that might be expected from an increase in feed raises the temperature and pressure of the plasma, causing it to expand against the confining magnetic field. This expansion decreases the plasma density. The density decrease lowers the 
TABLE 8. Potential Initiating Mechanisms for

Accidents for Conceptual Fusion Reactor

Internal to Plant

1. Pipe Rupture

2. Pipe Blockage

3. Pump Failure

4. Change in Magnetic Field

5. Change in Plasma Feed Rate

6. Plasma Instabilities

7. Failure of He Containment Boundary

8. Radiation Damage

9. Loss of Cooling System Serving Cryogenic Devices

10. Steam Generator Tube Failures

11. Intermediate Heat Exchanger Tube Failures

12. Human Errors

13. Pipe Whip

14. Failure of Power Feedback Control Systems

15. Mechanical Yield Strengths Exceeded, due to

a. Radiation Degradation

b. Forces from High Transient Electrical Fields

c. Pressure Differences Across Vacuum Boundaries

d. Excessive Thermal Cycling

16. Parting of Electrical Conductor

17. Magnet Coil Breakage

18. Failure of Pressure Relief Components

19. Overwhelming of Pressure Relief Systems

20. Dewar System Rupture

21. Mercury Vapor Pressures Grossly Exceeding Pump Cel1 Pressures

22. Power Loss

23. Staff Sabotage

External to Plant

1. Earthquake

2. Tornado

3. Flood

4. Missile Impact (Plane, Meteor)
5. Explosions, Blasts, Fires (Nearby Industries, Access Routes)

6. Loss of Offsite Power

7. Sabotage 


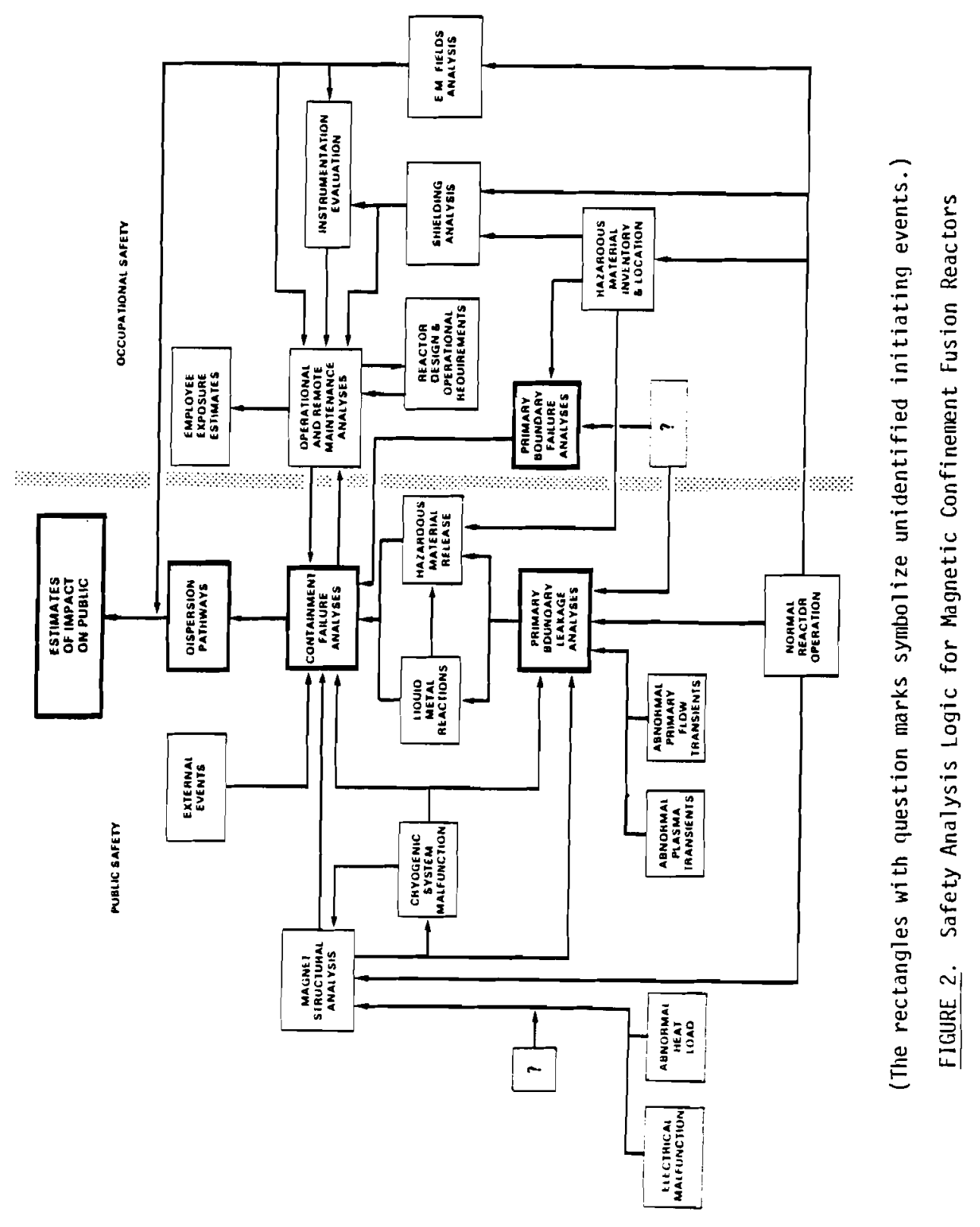


fusion reaction rate, making the plasma somewhat self-controlling. If the expanded plasma contacts the first wall, the reaction would immediately quench, since vaporized first wall material would enter the plasma and cool it to below the ignition temperature because of the greatly increased bremstralung radiation losses.

Changes in fuel feed, then appear at this time to be self-limiting. Damage to the first wall may or may not be extensive. Radioactive contaminants, tritium and some wall material may be released into the plasma confinement volume. However, containment integrity will not be affected. No releases of contaminants to the environs are expected to occur.

Plasma Instabilities. Plasma instabilities resulting in energy dumps in localized areas could result in first wall failure and fractional releases of the first wall, the blanket or coolant into the confinement volume. This loss of control could result from magnet failures or magnet instabilities or from loss of feedback control, (assuming feedback control from plasma temperature or other parameters will be used to adjust plasma feed rate). As discussed previously, first wall failure will quench the plasma reaction. Results of a dump of plasma kinetic energy to the first wall by an instability will be 1 imited to brief vaporization of wall or blanket material and release of tritium and other radioactive material into the confinement volume. No contaminants would be released from the containment vessel from this single incident.

Loss of Cooling. A loss of coolant accident, due to flow blockage or an actual loss, without prompt reactor shutdown has been analyzed to result in damage to the first wall.

Calculations ${ }^{(27)}$ based on the UWMAK I design description indicate that temperature increases in the first wall as much as $10^{\circ} \mathrm{K} / \mathrm{sec}$ could result if the plasma reaction were not terminated during a loss of coolant flow accident. First wall melting and failure could result from this accident and vaporization of $60-400 \mathrm{~kg}$ of the first wall material of a $5000 \mathrm{MWt}$ device has been postulated. (25) Most of this material would be expected to recondense rapidiy, however. Other calculations indicate melting temperatures are reached in shorter times at the divertor for this accident. (28)

As a single event, this is not considered to be a threat to the safety of the public. It should be noted that the plasma behavior in the confinement volume is completely decoupled from events in the wall coolant and blanket. No feedback to the plasma exists that could contribute to uncontrolled power increases in the plasma from accidents in the volume surrounding the outer wall.

\section{Consequences of Unit Source Terms}

Some insights can be gained into the safety significance of toxic materials contained in conceptual fusion power plants by reviewing the potential consequences of unit source terms. The purpose of this section is to provide this perspective for postulated acute (accidental) releases of selected radioactive materials. The releases or unit source terms assumed in the consequence calculation are not meant to reflect the potential magnitude of accidental releases expected from fusion reactors. The unit source terms with the corresponding consequences found in this section are intended merely to related potential radiation exposure to the public from releases of radioactive 
material. The consequences of these unit source terms can subsequently be scaled relative to the quantity released when potential accidental releases can be quantitatively defined.

A summary of the more relevant radioactive materials expected ${ }^{(29)}$ to be contained in fusion power plants is shown in Table 9 . Some of the information required in consequence calculations; namely, the organ effected by the intake of the radioisotope and the effective half life in that organ is also included. Consequence calculations of unit source terms, in terms of individual dose as a function of distance, were made for three major categories of these radioisotopes, tritium, corrosion products and activated air products. A summary of the input to the calculations reported in this section is shown in Table 10.

The computer codes used in calculating the dose to humans from the unit source terms were SUBDOSA ${ }^{(30)}$ and DACRIN. (31) SUBDOSA is used to calculate the dose from airborne radionuclides external to the body. DACRIN is used to calculate the dose resulting from radionuclides deposited in the body and its organs via inhalation. DACRIN uses the ICRP Task Group Lung Model (TGLM) to calculate the uptake of radioactive materials into the body and the resultant dose to various body organs.

The models contained in these computer codes relate doses as a function of: (1) quantity, physical form and type of material released, (2) duration of release, (3) atmospheric conditions during release, (4) horizontal distance from release point, and (5) release height. In these calculations, no consideration was made for plume buoyancy. Radioactive materials released as a result of a fire will rise as a function of heat input to the plume. When more detailed analyses are warranted, plume risk formulation should be factored into the dispersion calculations.

The results of the unit source term calculations for the relevant radioisotopes are summarized in Table 11 - A through E; Table 11-A, Dose Comitment from Ground Level Releases - Soluble Form; Table 11-B, Dose Commitment from Ground Level Releases - Insoluble Form; Table 11-C, Dose Commitment from $100 \mathrm{~m}$ Releases - Soluble Form; Table 11-0, Dose Commitment from $100 \mathrm{~m}$ Releases In soluble Form; Table $11-E$, External Dose from Ground Level and $100 \mathrm{~m}$ Releases. These results are graphically illustrated for tritium oxide, ${ }^{55} \mathrm{Fe}$, and ${ }^{95} \mathrm{Nb}$ in Figures 3 through 7 .

The unit source terms-consequence calculations of themselves do not reflect the relative public safety importance of the individual radioisotopes. Also necessary for an analysis is the concentration of the radioisotopes in the dispersible material at the time of release. To provide some perspective on this, the relative concentration of the relevant radioisotopes in the bianket structures for the theta-pinch, UWMAK 1, and PPPL reactors are presented in Table 12 . All activities shown are at shutdown following 5-10 years of operations. Short half-life and low energy radioisotopes are not shown in the table. By inspection of Table 11 and Table 12, it can be seen that ${ }^{95} \mathrm{Nb},{ }^{55} \mathrm{Fe}$, and ${ }^{57} \mathrm{Co}$ are the radioisotopes of the most significance in the blanket structure relative to the potential for public exposure. This assumes some potential for dispersability as collected products of corrosion or sputtering processes. 
TABLE 9. Physical, Biological and Effective Half Lives in the Human Body of Isotopes of Interest in Fusion Power

Plant Safety Studies (Data from Reference 29)

\begin{tabular}{|c|c|c|c|c|}
\hline \multirow[b]{2}{*}{ Isotope } & & \multicolumn{3}{|c|}{ Half Life (Days) } \\
\hline & Organ of Interest & Physical & Biological & Effective \\
\hline $3^{3}$ & Total Body & $4.5 \times 10^{3}$ & 12 & 10 \\
\hline${ }^{55} \mathrm{Fe}$ & Total Body & $1.1 \times 10^{3}$ & 800 & 463 \\
\hline & Spleen & & 600 & 388 \\
\hline & Lungs & & $3.2 \times 10^{3}$ & 819 \\
\hline${ }^{51} \mathrm{Cr}$ & Total Body & 27.8 & 616 & 26.6 \\
\hline & GI Tract & & 616 & 26.6 \\
\hline${ }^{57} \mathrm{Co}$ & Total Body & 270 & 9.5 & 9.2 \\
\hline${ }^{58} \mathrm{Co}$ & & 72 & & 8.4 \\
\hline${ }^{60} \mathrm{Co}$ & & $1.9 \times 10^{3}$ & & 9.5 \\
\hline $63_{\mathrm{Ni}}$ & Total Body & $2.9 \times 10^{4}$ & 667 & 652 \\
\hline & Bone & & 800 & 492 \\
\hline${ }^{54} \mathrm{Mn}$ & Total Body & 300 & 17 & 5.6 \\
\hline${ }^{95} \mathrm{Nb}$ & Total Body & 35 & 760 & 33.5 \\
\hline & Bone & & 1000 & 33.8 \\
\hline $93 m_{N b}$ & Total Body & $3.7 \times 10^{3}$ & 760 & 630 \\
\hline & Bone & & 1000 & 787 \\
\hline${ }^{14} \mathrm{C}$ & Total Body & $2 \times 10^{6}$ & 10 & 10 \\
\hline & Bone & & 40 & 40 \\
\hline${ }^{41} \mathrm{Ar}$ & Total Body & 0.076 & -- & $\cdots$ \\
\hline
\end{tabular}


TABLE 10. Unit Source Term - Consequence Calculations Input Parameters

\begin{tabular}{|c|c|c|c|c|c|}
\hline $\begin{array}{l}\text { Radioactive } \\
\text { Material } \\
\end{array}$ & $\begin{array}{l}\text { Chemical } \\
\text { Form } \\
\end{array}$ & $\begin{array}{l}\text { ReTease Quantity } \\
\text { Assumed, Ci } \\
\end{array}$ & $\begin{array}{l}\text { Quration of } \\
\text { Release, hrs }\end{array}$ & $\begin{array}{c}\text { Release } \\
\text { Helght, m }\end{array}$ & Exposure Pathivay \\
\hline \multicolumn{6}{|l|}{ Iritium: } \\
\hline $\bar{H}$ (oxide) & SOL $(1)$ & 1000 & $8^{(4)}$ & $100^{(5)}$ & INHAL ${ }^{(6)}$ \\
\hline \multicolumn{6}{|c|}{ Corrosion Products: } \\
\hline${ }^{51} \mathrm{Cr}$ & IHSOL $_{\text {SOL }}(2)$ & 1 & 8 & $100^{\circ}$ & INHAL, EXT ${ }^{(7)}$ \\
\hline${ }^{54_{\mathrm{Mn}}}$ & $\begin{array}{l}\text { SOL } \\
\text { INSOL }\end{array}$ & 1 & 8 & $\begin{array}{r}0 \\
100\end{array}$ & INHAL, EXT \\
\hline $555_{\mathrm{Fe}}$ & $\begin{array}{l}\text { SOL } \\
\text { INSOL }\end{array}$ & 1 & 8 & $\begin{array}{r}0 \\
100\end{array}$ & INHAL \\
\hline${ }^{57} \mathrm{Co}$ & $\begin{array}{l}\text { SOL } \\
\text { INSOL }\end{array}$ & 1 & 8 & $\begin{array}{r}0 \\
100\end{array}$ & INHAL, EXT \\
\hline${ }^{58} \mathrm{CO}$ & $\begin{array}{l}\text { SOL } \\
\text { INSOL }\end{array}$ & 1 & 8 & 100 & INHAL, EXT \\
\hline${ }^{60} \mathrm{Co}$ & $\begin{array}{l}\text { SOL } \\
\text { INSOL }\end{array}$ & 1 & 8 & 100 & INHAL, EXT \\
\hline${ }^{63}{ }_{\mathrm{Mi}}$ & $\begin{array}{l}\text { SOL } \\
\text { INSOL }\end{array}$ & 1 & 8 & $\begin{array}{r}0 \\
100\end{array}$ & INHAL \\
\hline $93 m_{i b}$ & $\begin{array}{l}\text { SOL } \\
\text { INSOL }\end{array}$ & 1 & 8 & $\begin{array}{r}0 \\
100\end{array}$ & EXT \\
\hline $95_{\mathrm{Nb}}$ & $\begin{array}{l}\text { SOL } \\
\text { INSOL }\end{array}$ & 1 & 8 & $\begin{array}{r}0 \\
100\end{array}$ & INHAL, EXT \\
\hline${ }^{14} \mathrm{C}$ & $\begin{array}{l}\text { SOL } \\
\text { IHISOI. }\end{array}$ & 1 & 8 & $\begin{array}{r}0 \\
100\end{array}$ & INHAL \\
\hline \multicolumn{6}{|c|}{ Activated Air Products: } \\
\hline $41_{\text {Ar }}$ & $\mathrm{NA}^{(3)}$ & 1 & 8 & $\begin{array}{r}0 \\
100\end{array}$ & EXT \\
\hline
\end{tabular}

(1) SOL means soluble, Translocation Class D.

(2) INSOL means insoluble. Translocation Class $Y$ except $14 \mathrm{C}$ which is $\mathrm{Class} \mathrm{W}$.

(3) NA means not applicable. Chemical form not relevant for external exposure.

(4) The 0-8 hour release curves from Regulatory Safety Guide No. 3 were used for weather conditions and dispersion.

(5) 0 means ground level release.

(6) INHAL means the exposure pathway was from intalation and subsequent deposition of the radioiso tope.

(7) EXT means the exposure pathway was from external radiation from the passing cloud. 
TABLE 11. Dose as a Function of Downwind Distance Following the Release

of Various Radioactive Substances Which may be Present in Fusion

\begin{tabular}{|c|c|c|c|c|c|c|c|c|c|c|c|c|c|}
\hline \multirow[b]{3}{*}{ Nuclide } & \multirow{3}{*}{$\begin{array}{l}\text { Reference } \\
\text { Organ }\end{array}$} & \multicolumn{11}{|c|}{$\begin{array}{l}\text { A. Dose Comnitment from Inhalation Following the } \\
\text { Release at Ground Level of } 1 \text { S Sized Particles of Various } \\
\text { Isotopes in Soluble Chemical Form. (Safety Guide } 30-8 \\
\text { hour Weather Condftions) }\end{array}$} & \multirow[b]{3}{*}{$\underline{100,000}$} \\
\hline & & \multirow{2}{*}{$\begin{array}{c}\text { Translocation } \\
\text { Class }\end{array}$} & \multirow{2}{*}{$\begin{array}{l}\text { Quantity } \\
\text { Released } \\
\text { (Ci) }\end{array}$} & \multicolumn{9}{|c|}{ Dose (rem) at Downwind Distance (m) } & \\
\hline & & & & 100 & 200 & 500 & 1000 & $\underline{2000}$ & $\underline{5000}$ & $\underline{10,000}$ & $\underline{20,000}$ & 50,000 & \\
\hline${ }^{3} \mathrm{H}$ & Total Body & $D$ & $10^{3}$ & 1.6 & $3.5-1$ & $0.6-2$ & $1.7-2$ & $6.0-3$ & $1.6-3$ & $6.4-4$ & $2.8-4$ & $9.6-5$ & $4 \cdot 5-5$ \\
\hline${ }^{114} \mathrm{C}$ & $\begin{array}{c}\text { Total Body } \\
\text { Bone }\end{array}$ & 0 & 1 & $\begin{array}{l}7.0-3 \\
3.5-2\end{array}$ & $\begin{array}{l}1.6-3 \\
7.8-3\end{array}$ & $\begin{array}{l}2.7-4 \\
1.4-3\end{array}$ & $\begin{array}{l}7.9-5 \\
3.9-4\end{array}$ & $\begin{array}{l}2.7-5 \\
1.4-4\end{array}$ & $\begin{array}{l}7.1-6 \\
3.6-5\end{array}$ & $\begin{array}{l}2.9-6 \\
1.4-5\end{array}$ & $\begin{array}{l}1.3-6 \\
6.4-6\end{array}$ & $\begin{array}{l}4.3-7 \\
4.3-7\end{array}$ & $\begin{array}{l}2.0-7 \\
2.0-7\end{array}$ \\
\hline s. Fe & $\begin{array}{l}\text { Total Body } \\
\text { Spleen }\end{array}$ & D & 1. & $\begin{array}{l}3.0-2 \\
2.4-1\end{array}$ & $\begin{array}{l}6.8-3 \\
5.3-2\end{array}$ & $\begin{array}{l}1.2-3 \\
9.2-3\end{array}$ & $\begin{array}{l}3.4-4 \\
2.7-3\end{array}$ & $\begin{array}{l}1.2-4 \\
9.1-4\end{array}$ & $\begin{array}{l}3.1-5 \\
2.4-4\end{array}$ & $\begin{array}{l}1.3-5 \\
9.9-5\end{array}$ & $\begin{array}{l}5.5-6 \\
4.3-5\end{array}$ & $\begin{array}{l}1.9-6 \\
1.5-5\end{array}$ & $\begin{array}{l}8.8-7 \\
6.9-6\end{array}$ \\
\hline${ }^{60} \mathrm{Co}$ & $\begin{array}{l}\text { Total Body } \\
\text { GI Tract }\end{array}$ & D & 1 & $\begin{array}{l}1.5-1 \\
2.0-1\end{array}$ & $\begin{array}{l}3.4-2 \\
4.5-2\end{array}$ & $\begin{array}{l}5.9-3 \\
7.8-3\end{array}$ & $\begin{array}{l}1.7-3 \\
2.3-3\end{array}$ & $\begin{array}{l}5.8-4 \\
7.8-4\end{array}$ & $\begin{array}{l}1.6-4 \\
2.1-4\end{array}$ & $\begin{array}{l}6.3-5 \\
8.4-5\end{array}$ & $\begin{array}{l}2.8-5 \\
3.7-5\end{array}$ & $\begin{array}{l}9.4-6 \\
1.2-5\end{array}$ & $\begin{array}{l}4.4-6 \\
5.8-6\end{array}$ \\
\hline $63^{3} \mathrm{i}$ & $\begin{array}{c}\text { Total Body } \\
\text { Bone }\end{array}$ & 0 & 1 & $\begin{array}{l}1.5-1 \\
4.6+0\end{array}$ & $\begin{array}{l}3.3-2 \\
1.0+0\end{array}$ & $\begin{array}{l}5.7-3 \\
1.8-1\end{array}$ & $\begin{array}{l}1.6-3 \\
5.2-2\end{array}$ & $\begin{array}{l}5.6-4 \\
1.8-2\end{array}$ & $\begin{array}{l}1.5-4 \\
4.7-3\end{array}$ & $\begin{array}{l}6.1-5 \\
1.9-3\end{array}$ & $\begin{array}{l}2.7-5 \\
8.4-4\end{array}$ & $\begin{array}{l}9.1-6 \\
2.8-4\end{array}$ & $\begin{array}{l}4.3-6 \\
1.3-4\end{array}$ \\
\hline $25 \mathrm{Nb}$ & $\begin{array}{c}\text { Total Body } \\
\text { Bone }\end{array}$ & 0 & 1 & $\begin{array}{l}1.6-1 \\
4.6-1\end{array}$ & $\begin{array}{l}3.7-2 \\
1.0-1\end{array}$ & $\begin{array}{l}6.4-3 \\
1.8-2\end{array}$ & $\begin{array}{l}1.9-3 \\
5.2-3\end{array}$ & $\begin{array}{l}6.4-4 \\
1.8-3\end{array}$ & $\begin{array}{l}1.7-4 \\
4.7-4\end{array}$ & $\begin{array}{l}6.8-5 \\
1.9-4\end{array}$ & $\begin{array}{l}3.0-5 \\
8.3-5\end{array}$ & $\begin{array}{l}1.0-5 \\
2.8 .5\end{array}$ & $\begin{array}{l}4.7-6 \\
1.3-5\end{array}$ \\
\hline${ }^{51} \mathrm{Cr}$ & $\begin{array}{l}\text { Total Body } \\
\text { GI Tract }\end{array}$ & D & 1 & $\begin{array}{l}6.4-3 \\
6.1-3\end{array}$ & $\begin{array}{l}1.4-3 \\
1.4-3\end{array}$ & $\begin{array}{l}2.5-4 \\
2.4-4\end{array}$ & $\begin{array}{l}7.2-5 \\
6.9-5\end{array}$ & $\begin{array}{l}2.5-5 \\
2.4-5\end{array}$ & $\begin{array}{l}6.6-6 \\
6.2-6\end{array}$ & $\begin{array}{l}2.7-6 \\
2.5-6\end{array}$ & $\begin{array}{l}1.2-6 \\
1.1-6\end{array}$ & $\begin{array}{l}3.9-7 \\
3.7-7\end{array}$ & $\begin{array}{l}1.8-7 \\
1.7-7\end{array}$ \\
\hline${ }^{51} M n$ & Total Body & D & 1 & $8.3-2$ & $1.8-2$ & $3.2-3$ & $9.3-4$ & $3.2-4$ & $8.4-5$ & $3.4-5$ & $1.5-5$ & $5.1-6$ & $2.4-6$ \\
\hline${ }^{5 \%} \mathrm{Co}$ & $\begin{array}{c}\text { Total Body } \\
\text { GI Tract }\end{array}$ & D & 1 & $\begin{array}{l}8.8-3 \\
1.6-2\end{array}$ & $\begin{array}{l}2.0-3 \\
3.9-3\end{array}$ & $\begin{array}{l}3.4-4 \\
6.9-4\end{array}$ & $\begin{array}{l}9.9-5 \\
2.0-4\end{array}$ & $\begin{array}{l}3.4-5 \\
6.8-5\end{array}$ & $\begin{array}{l}9.0-6 \\
1.8-5\end{array}$ & $\begin{array}{l}3.7-6 \\
7.3-6\end{array}$ & $\begin{array}{l}1.6-6 \\
3.2-6\end{array}$ & $\begin{array}{l}5.4-7 \\
1.1-6\end{array}$ & $\begin{array}{l}2.6-7 \\
5.1-7\end{array}$ \\
\hline${ }^{4} \mathrm{Co}$ & $\begin{array}{l}\text { Total Body } \\
\text { GI Tract }\end{array}$ & D & 1 & $\begin{array}{l}5.5-2 \\
7.8-2\end{array}$ & $\begin{array}{l}1.2-2 \\
1.7-2\end{array}$ & $\begin{array}{l}2.1-3 \\
3.0-3\end{array}$ & $\begin{array}{l}6.1-4 \\
8.8-4\end{array}$ & $\begin{array}{l}2.1-4 \\
3.0-4\end{array}$ & $\begin{array}{l}5.6-5 \\
8.0-5\end{array}$ & $\begin{array}{l}2.3-5 \\
3.2-5\end{array}$ & $\begin{array}{l}9.9-6 \\
1.4-5\end{array}$ & $\begin{array}{l}3.4-6 \\
4.8-6\end{array}$ & $\begin{array}{l}1.6-6 \\
2.2-6\end{array}$ \\
\hline
\end{tabular}

(a) $1.6+0=1.6 \times 10^{1}$ 
TABLE 11. (Contd)

B. Dose Comilitment from Inhalation following the

Release at Ground Level of $1 \mu$ Sized Particles of Various

Isotopes in Insoluble Chemical Form (Safety Guide 3 0-8

hour Weather Conditions

\begin{tabular}{|c|c|c|c|c|c|c|c|c|c|c|c|c|c|}
\hline \multirow[b]{2}{*}{ Nuclide } & \multirow{2}{*}{$\begin{array}{c}\text { Reference } \\
\text { Organ }\end{array}$} & \multirow{2}{*}{$\begin{array}{c}\text { Translocat ion } \\
\text { class } \\
\end{array}$} & \multirow{2}{*}{$\begin{array}{l}\text { Quantity } \\
\text { Released } \\
\text { (Ci) }\end{array}$} & \multicolumn{10}{|c|}{ Dose (rem) at Downwind Distance (m) } \\
\hline & & & & 100 & 200 & 500 & 1000 & 2000 & 5000 & 10,000 & 20,000 & 50,000 & 100,000 \\
\hline${ }^{1 /,} \mathrm{C}$ & $\begin{array}{l}\text { Total Body } \\
\text { Bone } \\
\text { Lungs }\end{array}$ & W & 1 & $\begin{array}{l}7.0-3^{(a)} \\
3.5-2 \\
7.0-3\end{array}$ & $\begin{array}{l}1.6-3 \\
7.8-3 \\
1.6-3\end{array}$ & $\begin{array}{l}2.7-4 \\
1.4-3 \\
2.7-4\end{array}$ & $\begin{array}{l}7.9-5 \\
3.9-4 \\
7.9-5\end{array}$ & $\begin{array}{l}2.7-5 \\
1.4-4 \\
2.7-5\end{array}$ & $\begin{array}{l}7.1-6 \\
3.6-5 \\
7.1-6\end{array}$ & $\begin{array}{l}2.9-6 \\
1.4-5 \\
2.9-6\end{array}$ & $\begin{array}{l}1.3-6 \\
6.4-6 \\
1.3-6\end{array}$ & $\begin{array}{l}4.3-7 \\
2.2-6 \\
4.3-7\end{array}$ & $\begin{array}{l}2.0-7 \\
1.0-6 \\
2.0-7\end{array}$ \\
\hline stse & $\begin{array}{l}\text { Total Body } \\
\text { Spleen } \\
\text { Lungs }\end{array}$ & Y & 1 & $\begin{array}{l}4.9-3 \\
3.8-2 \\
4.9-1\end{array}$ & $\begin{array}{l}1.1-3 \\
8.5-3 \\
1.1-1\end{array}$ & $\begin{array}{l}1.9-4 \\
1.5-3 \\
1.9-2\end{array}$ & $\begin{array}{l}5.5-5 \\
4.2-4 \\
5.5-3\end{array}$ & $\begin{array}{l}1.9-5 \\
1.5-4 \\
1.9-3\end{array}$ & $\begin{array}{l}5.0-6 \\
3.9-5 \\
5.0-4\end{array}$ & $\begin{array}{l}2.0-6 \\
1.6-5 \\
2.0-4\end{array}$ & $\begin{array}{l}8.9-7 \\
6.9-6 \\
8.8-5\end{array}$ & $\begin{array}{l}3.0-7 \\
2.4-6 \\
3.0-5\end{array}$ & $\begin{array}{l}1.4-7 \\
1.1-6 \\
1.4-5\end{array}$ \\
\hline${ }^{60} \mathrm{Co}$ & $\begin{array}{c}\text { Total Body } \\
\text { GI Tract } \\
\text { Lungs }\end{array}$ & Y & 1 & $\begin{array}{l}5.8-2 \\
7.2-1 \\
6.0+1\end{array}$ & $\begin{array}{l}1.3-2 \\
1.6-1 \\
1.4+1\end{array}$ & $\begin{array}{l}2.2-3 \\
2.8-2 \\
2.4+0\end{array}$ & $\begin{array}{l}6.5-4 \\
8.1-3 \\
6.8-1\end{array}$ & $\begin{array}{l}2.2-4 \\
2.8-3 \\
2.3-1\end{array}$ & $\begin{array}{l}5.9-5 \\
7.4-4 \\
6.2-2\end{array}$ & $\begin{array}{l}2.4-5 \\
3.0-4 \\
2.5-2\end{array}$ & $\begin{array}{l}1.0-5 \\
1.3-4 \\
1.1-2\end{array}$ & $\begin{array}{l}3.6-6 \\
4.5-5 \\
3.7-3\end{array}$ & $\begin{array}{l}1.7-6 \\
2.1-5 \\
1.8-3\end{array}$ \\
\hline $1{ }^{3} \mathrm{Ni}$ & $\begin{array}{c}\text { Total Body } \\
\text { Bone } \\
\text { Lungs }\end{array}$ & Y & 1 & $\begin{array}{l}6.2-2 \\
1.9+0 \\
2.2+0\end{array}$ & $\begin{array}{l}1.4-2 \\
4.3-1 \\
4.9-1\end{array}$ & $\begin{array}{l}2.4-3 \\
7.5-2 \\
8.5-2\end{array}$ & $\begin{array}{l}7.0-4 \\
2.2-2 \\
2.5-2\end{array}$ & $\begin{array}{l}2.4-4 \\
7.4-3 \\
8.4-3\end{array}$ & $\begin{array}{l}6.3-5 \\
2.0-3 \\
2.2-3\end{array}$ & $\begin{array}{l}2.6-5 \\
8.0-4 \\
9.1-4\end{array}$ & $\begin{array}{l}1.1-5 \\
3.5-4 \\
4.0-4\end{array}$ & $\begin{array}{l}3.8-6 \\
1.2-4 \\
1.4-4\end{array}$ & $\begin{array}{l}1.8-6 \\
5.6-5 \\
6.4-5\end{array}$ \\
\hline $45 \mathrm{Nb}$ & $\begin{array}{c}\text { Total Body } \\
\text { Bone } \\
\text { Lungs }\end{array}$ & Y & 1 & $\begin{array}{l}1.7-3 \\
4.7-3 \\
1.8+0\end{array}$ & $\begin{array}{l}3.7-4 \\
1.0-3 \\
4.1-1\end{array}$ & $\begin{array}{l}6.5-5 \\
1.8-4 \\
7.1-2\end{array}$ & $\begin{array}{l}1.9-5 \\
5.2-5 \\
2.1-2\end{array}$ & $\begin{array}{l}6.4-6 \\
1.8-5 \\
7.1-3\end{array}$ & $\begin{array}{l}1.7-6 \\
4.8-6 \\
1.9-3\end{array}$ & $\begin{array}{l}6.9-7 \\
1.9-6 \\
7.6-4\end{array}$ & $\begin{array}{l}3.0-7 \\
8.4-7 \\
3.3-4\end{array}$ & $\begin{array}{l}1.0-7 \\
2.8-7 \\
1.1-4\end{array}$ & $\begin{array}{l}4.8-8 \\
1.3-7 \\
5.2-5\end{array}$ \\
\hline sCr & $\begin{array}{c}\text { Total Body } \\
\text { Lungs }\end{array}$ & Y & 1 & $\begin{array}{l}9.5-5 \\
8.0-2\end{array}$ & $\begin{array}{l}2.1-5 \\
1.8-2\end{array}$ & $\begin{array}{l}3.7-6 \\
3.1-3\end{array}$ & $\begin{array}{l}1.1-6 \\
9.6-4\end{array}$ & $\begin{array}{l}3.6-7 \\
3.1-4\end{array}$ & $\begin{array}{l}9.6-8 \\
8.2-5\end{array}$ & $\begin{array}{l}3.9-8 \\
3.3-5\end{array}$ & $\begin{array}{l}1.7-8 \\
1.4-5\end{array}$ & $\begin{array}{l}5.8-9 \\
4.9-6\end{array}$ & $\begin{array}{l}2.8-9 \\
2.3-6\end{array}$ \\
\hline $5 \mathrm{Mn}$ & $\begin{array}{l}\text { Total Body } \\
\text { Lungs }\end{array}$ & Y & 1 & $\begin{array}{l}1.1-2 \\
9.2+0\end{array}$ & $\begin{array}{l}2.4-3 \\
2.0+0\end{array}$ & $\begin{array}{l}4.1-4 \\
3.6-1\end{array}$ & $\begin{array}{l}1.2-4 \\
1.0-1\end{array}$ & $\begin{array}{l}4.1-5 \\
3.5-2\end{array}$ & $\begin{array}{l}1.1-5 \\
9.3-3\end{array}$ & $\begin{array}{l}4.4-6 \\
3.8-3\end{array}$ & $\begin{array}{l}1.9-6 \\
1.7-3\end{array}$ & $\begin{array}{l}6.5-7 \\
5.6-4\end{array}$ & $\begin{array}{l}3.1-7 \\
2.6-4\end{array}$ \\
\hline $5.7 \mathrm{Co}$ & $\begin{array}{c}\text { Total Body } \\
\text { Lungs }\end{array}$ & Y & 1 & $\begin{array}{l}2.8-3 \\
2.0+0\end{array}$ & $\begin{array}{l}6.2-4 \\
4.4-1\end{array}$ & $\begin{array}{l}1.1-4 \\
7.7-2\end{array}$ & $\begin{array}{l}3.1-5 \\
2.2-2\end{array}$ & $\begin{array}{l}1.1-5 \\
7.4-3\end{array}$ & $\begin{array}{l}2.8-6 \\
2.0-3\end{array}$ & $\begin{array}{l}1.2-6 \\
8.2-4\end{array}$ & $\begin{array}{l}5.1-7 \\
3.6-4\end{array}$ & $\begin{array}{l}1.7-7 \\
1.2-4\end{array}$ & $\begin{array}{l}8.1-8 \\
5.7-5\end{array}$ \\
\hline${ }^{5 H} \mathrm{CO}$ & $\begin{array}{c}\text { Total Body } \\
\text { Lungs }\end{array}$ & Y & 1 & $\begin{array}{l}1.6-2 \\
3.9+0\end{array}$ & $\begin{array}{l}3.6-3 \\
8.7-1\end{array}$ & $\begin{array}{l}6.2-4 \\
1.5-1\end{array}$ & $\begin{array}{l}1.8-4 \\
4.4-2\end{array}$ & $\begin{array}{l}6.2-5 \\
1.5-2\end{array}$ & $\begin{array}{l}1.6-5 \\
4.0-3\end{array}$ & $\begin{array}{l}6.3-5 \\
1.6-3\end{array}$ & $\begin{array}{l}2.9-6 \\
7.1-4\end{array}$ & $\begin{array}{l}9.8-7 \\
2.4-4\end{array}$ & $\begin{array}{l}4.6-7 \\
1.1-4\end{array}$ \\
\hline
\end{tabular}

(a) $7.0-3: 7.0 \times 10^{-3}$ 
TABLE 11. (Contd)

C. Dose Commitment from Inhalation Following the

Release from a theight of $100 \mathrm{~m}$ of $1 \mu$ Sized Particles of

Various Isotopes in Soluble Chemical Form. (Safety Guide 3 0-8 hour Weather Conditions)

\begin{tabular}{|c|c|c|c|c|c|c|c|c|c|c|c|c|c|}
\hline \multirow[b]{2}{*}{ Nuclide } & \multirow{2}{*}{$\begin{array}{c}\text { Reference } \\
\text { Organ }\end{array}$} & \multirow{2}{*}{$\begin{array}{c}\text { Translocation } \\
\text { Class }\end{array}$} & \multirow{2}{*}{$\begin{array}{l}\text { Quantity } \\
\text { Released } \\
\text { (Ci) }\end{array}$} & \multicolumn{10}{|c|}{ Dose (rem) at Downwind Distance (m) } \\
\hline & & & & 100 & 200 & 500 & 1000 & $\underline{2000}$ & $\underline{5000}$ & $\underline{10,000}$ & $\underline{20,000}$ & $\underline{50,000}$ & $\underline{100,000}$ \\
\hline${ }^{3} \mathrm{H}$ & Total Body & 0 & $10^{3}$ & 0. & $2.0-5$ & $5.6-4$ & $4,0-4$ & $2.9-4$ & $1.8-4$ & $1.2-4$ & $8.0-5$ & $4.0-5$ & $2.2-5$ \\
\hline${ }^{14} \mathrm{C}$ & $\begin{array}{c}\text { Total Body } \\
\text { Bone }\end{array}$ & D & 1 & $\begin{array}{l}0 . \\
0 .\end{array}$ & $\begin{array}{l}9.2-8 \\
4.6-7\end{array}$ & $\begin{array}{l}2.5-6 \\
1.3-5\end{array}$ & $\begin{array}{l}1.8-6 \\
9.0-6\end{array}$ & $\begin{array}{l}1.3-6 \\
6.6-6\end{array}$ & $\begin{array}{l}8.2-7 \\
4.1-6\end{array}$ & $\begin{array}{l}5.6-7 \\
2.8-6\end{array}$ & $\begin{array}{l}3.6-7 \\
1.8-6\end{array}$ & $\begin{array}{l}1.8-7 \\
9.0-7\end{array}$ & $\begin{array}{l}9.8-8 \\
4.9-7\end{array}$ \\
\hline${ }^{55} \mathrm{Fe}$ & $\begin{array}{l}\text { Total Body } \\
\text { Spleen }\end{array}$ & 0 & 1 & $\begin{array}{l}0 . \\
0 .\end{array}$ & $\begin{array}{l}4.0-7 \\
3.1-6\end{array}$ & $\begin{array}{l}1.1-5 \\
8.6-5\end{array}$ & $\begin{array}{l}7.8-6 \\
6.1-5\end{array}$ & $\begin{array}{l}5.7-6 \\
4.5-5\end{array}$ & $\begin{array}{l}3.5-6 \\
2.8-5\end{array}$ & $\begin{array}{l}2.4-6 \\
1.9-5\end{array}$ & $\begin{array}{l}1.6-6 \\
1.2-5\end{array}$ & $\begin{array}{l}7.8-7 \\
6.1-6\end{array}$ & $\begin{array}{l}4.3-7 \\
3.3-6\end{array}$ \\
\hline${ }^{60} \mathrm{Co}$ & $\begin{array}{l}\text { Total Body } \\
\text { GI Tract }\end{array}$ & D & 1 & $\begin{array}{l}0 . \\
0 .\end{array}$ & $\begin{array}{l}2.0-6 \\
2.7-6\end{array}$ & $\begin{array}{l}5.5-5 \\
7.3-5\end{array}$ & $\begin{array}{l}3.9-5 \\
5.2-5\end{array}$ & $\begin{array}{l}2.9-5 \\
3.8-5\end{array}$ & $\begin{array}{l}1.8-5 \\
2.4-5\end{array}$ & $\begin{array}{l}1.2-5 \\
1.6-5\end{array}$ & $\begin{array}{l}7.8-6 \\
1.0-5\end{array}$ & $\begin{array}{l}3.9-6 \\
5.2-6\end{array}$ & $\begin{array}{l}2.1-6 \\
2.8-6\end{array}$ \\
\hline${ }^{63} \mathrm{Ni}$ & $\begin{array}{c}\text { Total Body } \\
\text { Bone }\end{array}$ & 0 & 1 & $\begin{array}{l}0 . \\
0 .\end{array}$ & $\begin{array}{l}1.9-6 \\
6.1-5\end{array}$ & $\begin{array}{l}5.3-5 \\
1.6-3\end{array}$ & $\begin{array}{l}3.8-5 \\
1.2-3\end{array}$ & $\begin{array}{l}2.8-5 \\
8.7-4\end{array}$ & $\begin{array}{l}1.7-5 \\
5.4-4\end{array}$ & $\begin{array}{l}1.2-5 \\
3.7-4\end{array}$ & $\begin{array}{l}7.6-6 \\
2.4-4\end{array}$ & $\begin{array}{l}3.8-6 \\
1.2-4\end{array}$ & $\begin{array}{l}2.1-6 \\
6.5-5\end{array}$ \\
\hline${ }^{95} \mathrm{Nb}$ & $\begin{array}{c}\text { Total Body } \\
\text { Bone }\end{array}$ & D & 1 & $\begin{array}{l}0 . \\
0 .\end{array}$ & $\begin{array}{l}2.2-6 \\
6.1-6\end{array}$ & $\begin{array}{l}6.0-5 \\
1.7-4\end{array}$ & $\begin{array}{l}4.2-5 \\
1.2-4\end{array}$ & $\begin{array}{l}3.1-5 \\
8.7-5\end{array}$ & $\begin{array}{l}1.9-5 \\
5.4-5\end{array}$ & $\begin{array}{l}1.3-5 \\
3.7-5\end{array}$ & $\begin{array}{l}8.5-6 \\
2.4-5\end{array}$ & $\begin{array}{l}4.2-6 \\
1.2-5\end{array}$ & $\begin{array}{l}2.3-6 \\
6.3-6\end{array}$ \\
\hline${ }^{51} \mathrm{Cr}$ & $\begin{array}{l}\text { Total Body } \\
\text { GI Tract }\end{array}$ & 0 & 1 & $\begin{array}{l}0 . \\
0 .\end{array}$ & $\begin{array}{l}8.5-8 \\
8.1-8\end{array}$ & $\begin{array}{l}2.3-6 \\
2.2-6\end{array}$ & $\begin{array}{l}1.7-6 \\
1.6-6\end{array}$ & $\begin{array}{l}1.2-6 \\
1.2-6\end{array}$ & $\begin{array}{l}7.5-7 \\
7.2-7\end{array}$ & $\begin{array}{l}5.2-7 \\
4.9-7\end{array}$ & $\begin{array}{l}3.3-7 \\
3.1-7\end{array}$ & $\begin{array}{l}1.6-7 \\
1.6-7\end{array}$ & $\begin{array}{l}8.8-8 \\
8.4-8\end{array}$ \\
\hline${ }^{5}{ }^{1} \mathrm{Mn}$ & Total Body & 0 & 1 & 0. & $1.1-6$ & $3.0-5$ & $2.1-5$ & $1.6-5$ & $9.6-6$ & $6.7-6$ & $4.2-6$ & $2.1-6$ & $1.2-6$ \\
\hline${ }^{57} \mathrm{Co}$ & $\begin{array}{l}\text { Total Body } \\
\text { GI Tract }\end{array}$ & $D$ & 1 & $\begin{array}{l}0 . \\
0 .\end{array}$ & $\begin{array}{l}1.2-7 \\
2.3-7\end{array}$ & $\begin{array}{l}3.2-6 \\
6.4-6\end{array}$ & $\begin{array}{l}2.3-6 \\
4.6-6\end{array}$ & $\begin{array}{l}1.7-6 \\
3.3-6\end{array}$ & $\begin{array}{l}1.0-6 \\
2.1-6\end{array}$ & $\begin{array}{l}7.1-7 \\
1.4-6\end{array}$ & $\begin{array}{l}4.6-7 \\
9.1-7\end{array}$ & $\begin{array}{l}2.3-7 \\
4.6-7\end{array}$ & $\begin{array}{l}1.2-7 \\
2.5-7\end{array}$ \\
\hline${ }^{58} \mathrm{Co}$ & $\begin{array}{l}\text { Total Body } \\
\text { GI Tract }\end{array}$ & D & 1 & $\begin{array}{l}0 . \\
0 .\end{array}$ & $\begin{array}{l}7.2-7 \\
1.0-6\end{array}$ & $\begin{array}{l}2.0-5 \\
2.8-5\end{array}$ & $\begin{array}{l}1.4-5 \\
2.0-5\end{array}$ & $\begin{array}{l}1.0-5 \\
1.5-5\end{array}$ & $\begin{array}{l}6.4-6 \\
9.1-6\end{array}$ & $\begin{array}{l}4.4-6 \\
6.4-6\end{array}$ & $\begin{array}{l}2.8-6 \\
4.0-6\end{array}$ & $\begin{array}{l}1.4-6 \\
2.0-6\end{array}$ & $\begin{array}{l}7.6-7 \\
1.1-6\end{array}$ \\
\hline
\end{tabular}

(a) $2.0-4 \equiv 2.0 \times 10^{-4}$ 
TABLE 11. (Contd)

D. Dose Comnitment from Inlalation Following the

Release From a Height of $100 \mathrm{~m}$ of $1 \mu$ Sized Particles of

Various Isotopes in Insoluble Chemical Form. (Safety

Guide 3 0-8 hour Weather Conditions)

\begin{tabular}{|c|c|c|c|c|c|c|c|c|c|c|c|c|c|}
\hline \multirow[b]{2}{*}{ Nuclide } & \multirow{2}{*}{$\begin{array}{l}\text { Reference } \\
\text { Organ }\end{array}$} & \multirow{2}{*}{$\begin{array}{c}\text { Translocation } \\
\text { Class } \\
\end{array}$} & \multirow{2}{*}{$\begin{array}{l}\text { Quantity } \\
\text { Released } \\
\text { (Ci) } \\
\end{array}$} & \multicolumn{10}{|c|}{ Dose (rem) at Downwind Distance (m) } \\
\hline & & & & 100 & 200 & 500 & 1000 & 2000 & 5000 & 10,000 & 20,000 & $\underline{50,000}$ & $\underline{100,000}$ \\
\hline${ }^{14} \mathrm{C}$ & $\begin{array}{c}\text { Total 8ody } \\
\text { Bone } \\
\text { Lungs }\end{array}$ & W & 1 & $\begin{array}{l}0 . \\
0 . \\
0 .\end{array}$ & $\begin{array}{l}9.2-8^{(a)} \\
4.6-7 \\
9.2-8\end{array}$ & $\begin{array}{l}2.5-6 \\
1.3-5 \\
2.5-6\end{array}$ & $\begin{array}{l}1.8-6 \\
9.0-6 \\
1.8-6\end{array}$ & $\begin{array}{l}1.3-6 \\
6.6-6 \\
1.3-6\end{array}$ & $\begin{array}{l}8.2-7 \\
4.7-6 \\
8.2-7\end{array}$ & $\begin{array}{l}5.6-7 \\
2.8-6 \\
5.6-7\end{array}$ & $\begin{array}{l}3.6-7 \\
1.8-6 \\
3.6-7\end{array}$ & $\begin{array}{l}1.8-7 \\
9.0-7 \\
1.8-7\end{array}$ & $\begin{array}{l}9.8-8 \\
4.9-7 \\
9.8-8\end{array}$ \\
\hline${ }^{5} \mathrm{Fe}$ & $\begin{array}{c}\text { Total Body } \\
\text { Spleen } \\
\text { Lungs }\end{array}$ & $Y$ & 1 & $\begin{array}{l}0 . \\
0 . \\
0 .\end{array}$ & $\begin{array}{l}6.4-8 \\
5.0-7 \\
6.4-6\end{array}$ & $\begin{array}{l}1.8-6 \\
1.4-5 \\
1.8-4\end{array}$ & $\begin{array}{l}1.3-6 \\
9.8-6 \\
1.2-4\end{array}$ & $\begin{array}{l}9.2-7 \\
7.2-6 \\
9.2-5\end{array}$ & $\begin{array}{l}5.7-7 \\
4.4-6 \\
5.7-5\end{array}$ & $\begin{array}{l}3.9-7 \\
3.1-6 \\
3.9-5\end{array}$ & $\begin{array}{l}2.5-7 \\
2.0-6 \\
2.5-5\end{array}$ & $\begin{array}{l}1.3-7 \\
9.8-7 \\
1.2-5\end{array}$ & $\begin{array}{l}6.9-8 \\
5.4-7 \\
6.8-6\end{array}$ \\
\hline${ }^{60} \mathrm{Co}$ & $\begin{array}{c}\text { Total Body } \\
\text { GI Tract } \\
\text { Lungs }\end{array}$ & Y & 1 & $\begin{array}{l}0 . \\
0 . \\
0 .\end{array}$ & $\begin{array}{l}7.6-7 \\
9.6-6 \\
7.0-4\end{array}$ & $\begin{array}{l}2.1-5 \\
2.6-4 \\
2.2-2\end{array}$ & $\begin{array}{l}1.5-5 \\
1.9-4 \\
1.6-2\end{array}$ & $\begin{array}{l}1.1-5 \\
1.4-4 \\
1.1-2\end{array}$ & $\begin{array}{l}6.7-6 \\
8.5-5 \\
7.0-3\end{array}$ & $\begin{array}{l}4.6-6 \\
5.8-5 \\
4.9-3\end{array}$ & $\begin{array}{l}3.0-6 \\
3.7-5 \\
3.1-3\end{array}$ & $\begin{array}{l}1.5-6 \\
1.9-5 \\
1.6-3\end{array}$ & $\begin{array}{l}8.1-7 \\
1.0-5 \\
8.5-4\end{array}$ \\
\hline${ }^{63} \mathrm{Ni}$ & $\begin{array}{c}\text { Total Body } \\
\text { Bone } \\
\text { Lungs }\end{array}$ & Y & 1 & $\begin{array}{l}0 . \\
0 . \\
0 .\end{array}$ & $\begin{array}{l}8.2-7 \\
2.6-5 \\
2.9-5\end{array}$ & $\begin{array}{l}2.2-5 \\
7.0-4 \\
7.9-4\end{array}$ & $\begin{array}{l}1.6-5 \\
5.0-4 \\
5.6-4\end{array}$ & $\begin{array}{l}1.2-5 \\
3.6-4 \\
4.1-4\end{array}$ & $\begin{array}{l}7.2-6 \\
2.3-4 \\
2.6-4\end{array}$ & $\begin{array}{l}5.0-6 \\
1.6-4 \\
1.8-4\end{array}$ & $\begin{array}{l}3.2-6 \\
1.0-4 \\
1.1-4\end{array}$ & $\begin{array}{l}7.6-6 \\
5.0-5 \\
5.6-5\end{array}$ & $\begin{array}{l}8.7-7 \\
2.7-5 \\
3.1-5\end{array}$ \\
\hline${ }^{9} \mathrm{Nb}$ & $\begin{array}{c}\text { Total Body } \\
\text { Bone } \\
\text { Lungs }\end{array}$ & Y & 1 & $\begin{array}{l}0 . \\
0 . \\
0 .\end{array}$ & $\begin{array}{l}2.2-8 \\
6.2-8 \\
2.4-5\end{array}$ & $\begin{array}{l}6.0-7 \\
1.7-6 \\
6.6-4\end{array}$ & $\begin{array}{l}4.3-7 \\
1.2-6 \\
4.7-4\end{array}$ & $\begin{array}{l}3.2-7 \\
8.8-7 \\
3.5-4\end{array}$ & $\begin{array}{l}2.0-7 \\
5.4-7 \\
2.7-4\end{array}$ & $\begin{array}{l}1.4-7 \\
3.8-7 \\
1.5-4\end{array}$ & $\begin{array}{l}8.6-8 \\
2.4-7 \\
9.4-5\end{array}$ & $\begin{array}{l}4.3-8 \\
1.2-7 \\
4.7-5\end{array}$ & $\begin{array}{l}2.3-8 \\
6.4-8 \\
2.5-5\end{array}$ \\
\hline${ }^{51} \mathrm{Cr}$ & $\begin{array}{c}\text { Total Body } \\
\text { Lungs }\end{array}$ & Y & 1 & 0. & $\begin{array}{l}1.2-9 \\
1.1-6\end{array}$ & $\begin{array}{l}3.4-8 \\
2.9-5\end{array}$ & $\begin{array}{l}2.4-8 \\
2.1-5\end{array}$ & $\begin{array}{l}1.8-8 \\
1.5-5\end{array}$ & $\begin{array}{l}1.1-8 \\
9.3-6\end{array}$ & $\begin{array}{l}7.6-9 \\
6.4-6\end{array}$ & $\begin{array}{l}4.8-9 \\
4.1-6\end{array}$ & $\begin{array}{l}2.4-9 \\
2.0-6\end{array}$ & $\begin{array}{l}1.3-9 \\
1.1-6\end{array}$ \\
\hline${ }^{5}{ }^{4} \mathrm{Mn}$ & $\begin{array}{c}\text { Total Body } \\
\text { Lungs }\end{array}$ & Y & 1 & $\begin{array}{l}0 . \\
0 .\end{array}$ & $\begin{array}{l}1.4-7 \\
1.2-4\end{array}$ & $\begin{array}{l}3.8-6 \\
3.3-3\end{array}$ & $\begin{array}{l}2.7-6 \\
2.4-3\end{array}$ & $\begin{array}{l}2.0-6 \\
1.7-3\end{array}$ & $\begin{array}{l}1.2-6 \\
1.1-3\end{array}$ & $\begin{array}{l}8.5-7 \\
7.4-4\end{array}$ & $\begin{array}{l}5.4-7 \\
4.7-4\end{array}$ & $\begin{array}{l}2.7-7 \\
2.4-4\end{array}$ & $\begin{array}{l}1.5-7 \\
1.3-4\end{array}$ \\
\hline${ }^{57} \mathrm{Co}$ & $\begin{array}{c}\text { Total 8ody } \\
\text { Lungs }\end{array}$ & Y & 1 & $\begin{array}{l}0 . \\
0 .\end{array}$ & $\begin{array}{l}3.7-8 \\
2.6-5\end{array}$ & $\begin{array}{l}1.0-6 \\
7.1-4\end{array}$ & $\begin{array}{l}7.2-7 \\
5.1-4\end{array}$ & $\begin{array}{l}5.3-7 \\
3.7-4\end{array}$ & $\begin{array}{l}3.3-7 \\
2.3-4\end{array}$ & $\begin{array}{l}2.2-7 \\
1.6-4\end{array}$ & $\begin{array}{l}1.4-7 \\
1.0-4\end{array}$ & $\begin{array}{l}7.2-8 \\
5.1-5\end{array}$ & $\begin{array}{l}3.9-8 \\
2.8-5\end{array}$ \\
\hline${ }^{58} \mathrm{Co}$ & $\begin{array}{c}\text { Total Body } \\
\text { Lungs }\end{array}$ & Y & 1 & $\begin{array}{l}0 . \\
0 .\end{array}$ & $\begin{array}{l}2.1-7 \\
5.2-5\end{array}$ & $\begin{array}{l}5.8-6 \\
1.4-3\end{array}$ & $\begin{array}{l}4.1-6 \\
1.0-3\end{array}$ & $\begin{array}{l}3.0-6 \\
7.4-4\end{array}$ & $\begin{array}{l}1.9-6 \\
4.6-4\end{array}$ & $\begin{array}{l}1.3-6 \\
3.2-4\end{array}$ & $\begin{array}{l}8.2-7 \\
2.0-4\end{array}$ & $\begin{array}{l}4.1-7 \\
1.0-4\end{array}$ & $\begin{array}{l}2.2-7 \\
5.4-5\end{array}$ \\
\hline
\end{tabular}

(a) $9.2-8 \equiv 9.2 \times 10^{-8}$ 
TABLE 11. (Contd)

E. External Dose Following the Release from Ground Level

and from a lleight of $100 \mathrm{~m}$ of $1 \mu$ Sized Particles of

Various Isotopes. (Safety Guide 3 0-8 hour Weather

Conditions) (I Ci Release for All Isotopes)

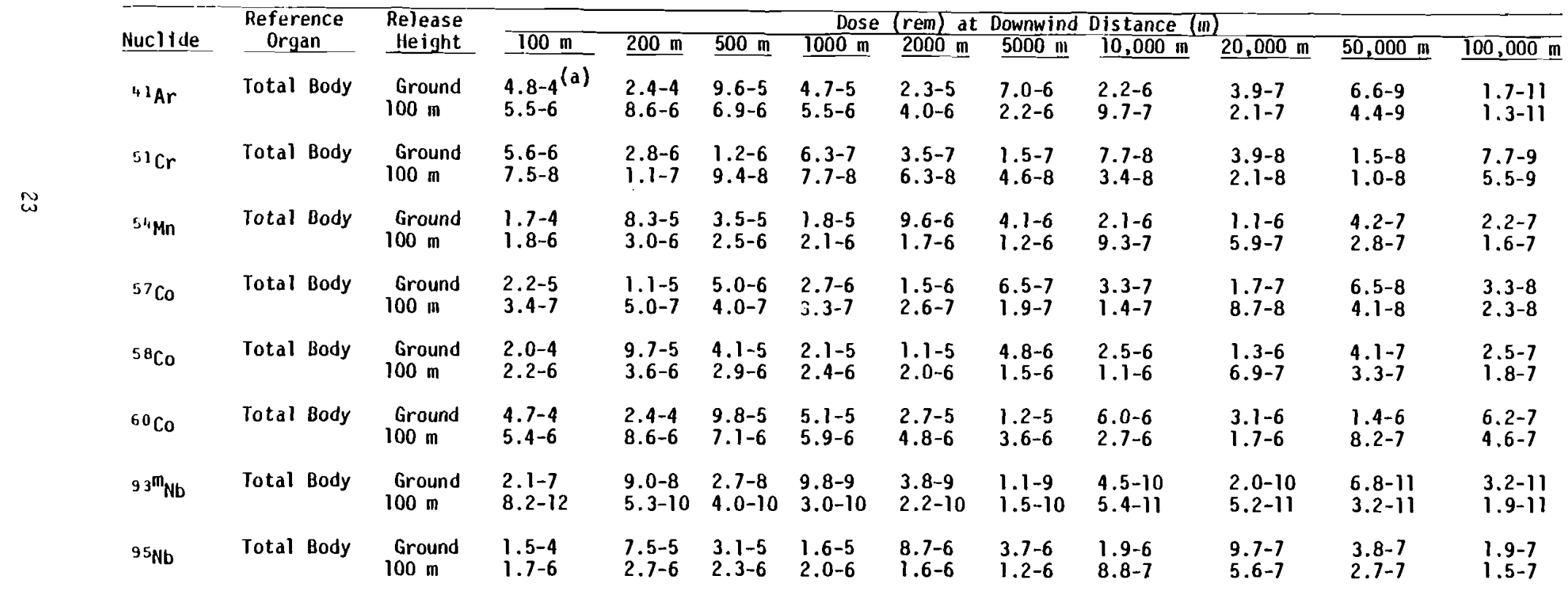

(a) $4.8-4 \equiv 4.8 \times 10^{-4}$ 

TABLE 12. Relative Concentration of Radioisotopes in Conceptual
Fusion Reactor Blankets $(16,23)$

\begin{tabular}{|c|c|c|c|}
\hline Reactor & Isotope & Activity (1) & Activity Fraction \\
\hline \multirow[t]{5}{*}{ Theta Pinch } & & $(C i / w t)$ & \\
\hline & ${ }^{95}{ }_{N_{b}}$ & 3 & 0.756 \\
\hline & $95 m_{N G}$ & 0.6 & 0.151 \\
\hline & $92 m_{i v b}$ & 0.3 & 0.075 \\
\hline & $93 m_{n b}$ & 0.07 & 0.018 \\
\hline
\end{tabular}

UWMAK I

\begin{tabular}{|c|c|c|}
\hline & (Ci) & \\
\hline${ }^{49} v$ & $1.110^{10}$ & 0.003 \\
\hline${ }^{51} \mathrm{Cr}$ & $4.2 \cdot 0^{11}$ & 0.109 \\
\hline${ }^{54} \mathrm{Mn}_{\mathrm{n}}$ & $4.010^{11}$ & 0.104 \\
\hline${ }^{55} \mathrm{Fe}$ & $2.310^{12}$ & 0.598 \\
\hline${ }^{57} \mathrm{Co}$ & $1.710^{11}$ & 0.044 \\
\hline $58 \mathrm{CO}$ & $4.710^{11}$ & 0.122 \\
\hline${ }^{60} \mathrm{Co}$. & $7.710^{10}$ & 0.020 \\
\hline
\end{tabular}

PPPL

\begin{tabular}{|c|c|c|}
\hline & (16i) & \\
\hline${ }^{3 /} \mathrm{CO}$ & $2.510^{9}$ & 0.573 \\
\hline${ }^{55} \mathrm{Fe}$ & $1.510^{9}$ & 0.344 \\
\hline${ }^{60} \mathrm{Co}$ & $1.610^{8}$ & 0.037 \\
\hline${ }^{54} \mathrm{Mn}$ & $1.610^{8}$ & 0.037 \\
\hline $63_{\mathrm{Ni}}$ & $3.010^{7}$ & 0.007 \\
\hline $49 v$ & $1.210^{7}$ & 0.003 \\
\hline
\end{tabular}

(1) Activities at shutdown following 5-10 years of operation.

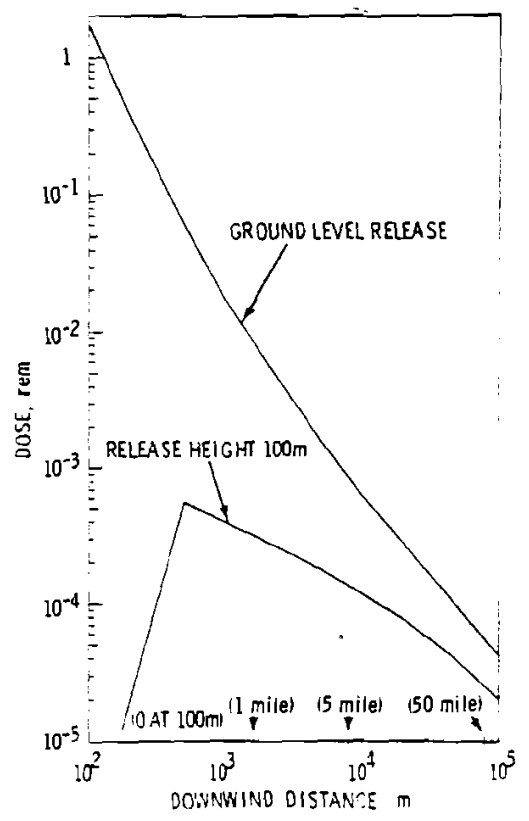

FIGURE 3. Total Body Jose as a Function of Distance Following the Release Over an 8-Hour Period of $1000 \mathrm{Ci}$ of Tritium as an 0xide 


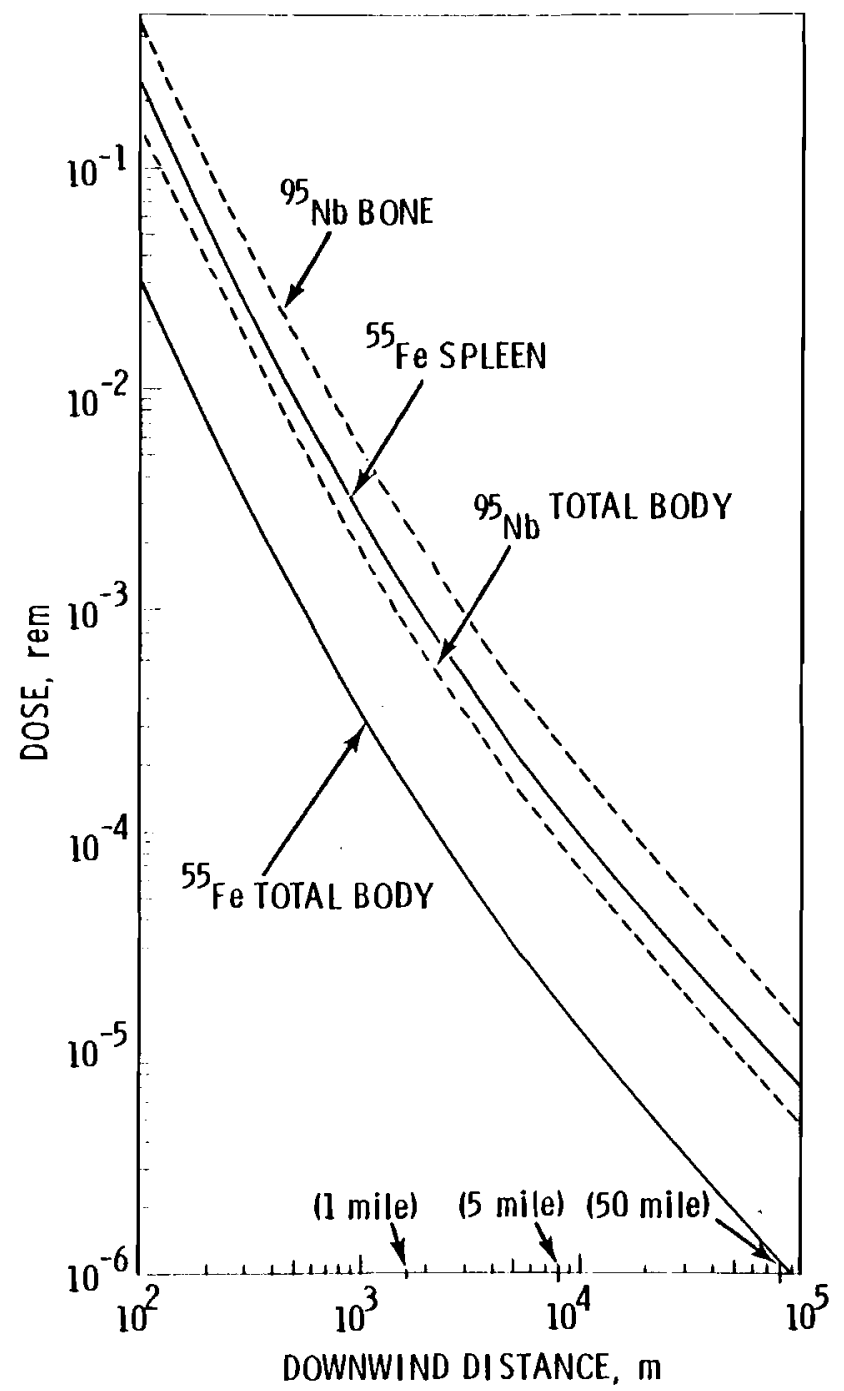

FIGIIRE 4. Inhalation Dose Versus Downwind Distance for a $1 \mathrm{Ci}$ Release at Ground fevel of 1 "Sized oarticles of $95 \mathrm{Nb}$ and $55 \mathrm{Fe}$ in Soluble Form

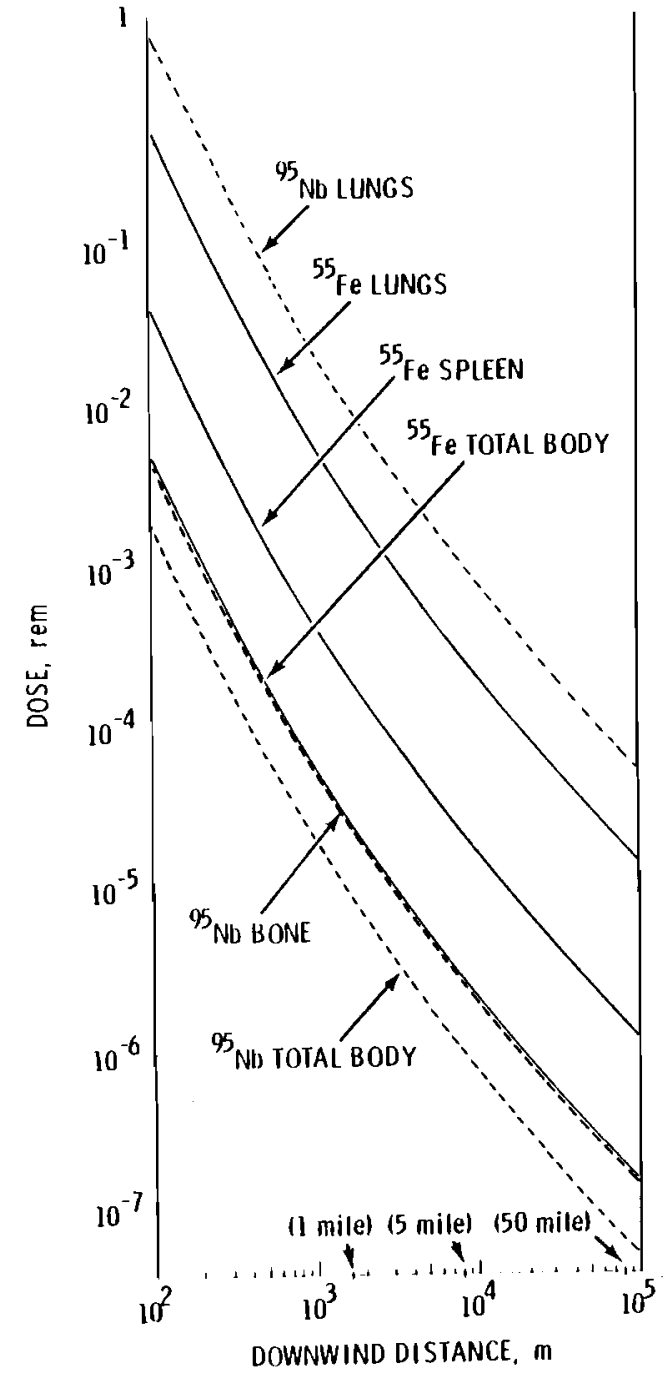

FIGURE 5. Inhalation Dose Versus Downwind Distance for a 1 Ci Release at Ground Level of $1 \mu$ Sized Particles of $95 \mathrm{Nb}$ and $55 \mathrm{Fe}$ in Insoluble Form 


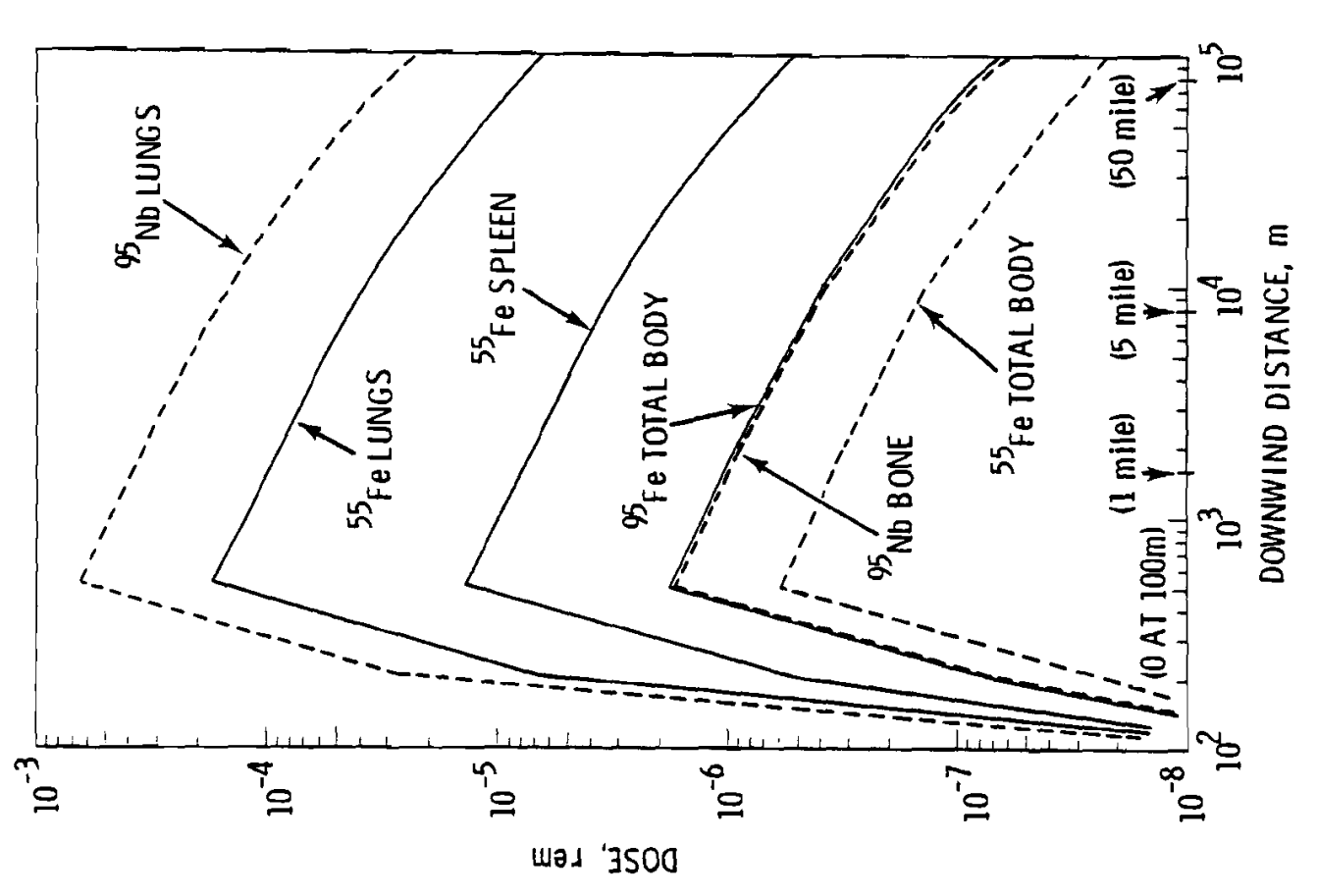

要

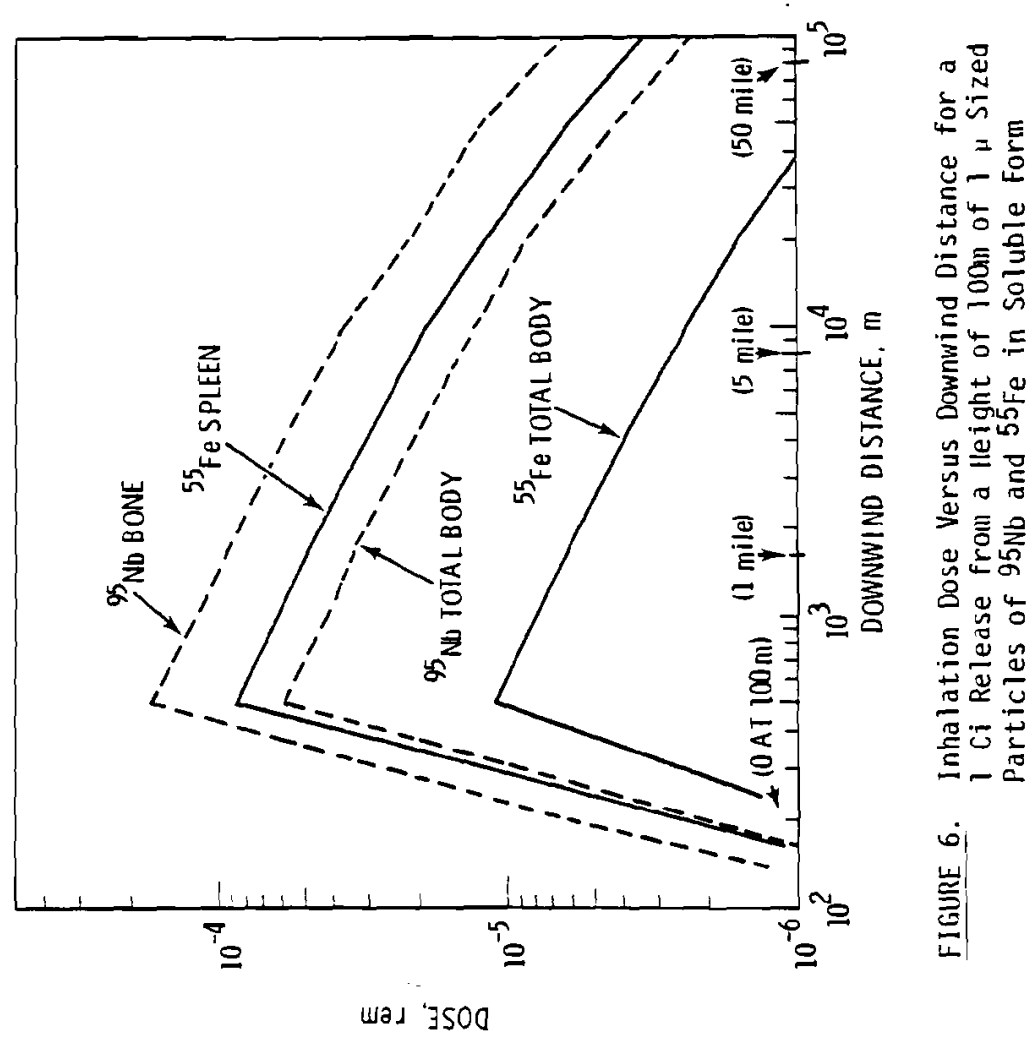


In addition to the scaling utility of the unit source term calculations, the results can be of value to the fusion reactor designer. Design alternatives and/or structural materials can be selected with prior knowledge of the more significant dose contributors in potential accidents. The dose information in Table 11, coupled with knowledge of dispersible toxic material inventories in conceptual fusion reactors, provides the designer with insights on where design emphasis is needed.

\section{RESEARCH AND DEVELOPMENT NEEDS}

There are technological areas where investment in research and development (R\&D) would lead to a better understanding of the potential safety implications of operating fusion power plants. It was therefore a secondary objective of the environmental analysis to identify areas where additional safety related experimental data or analytical methods are needed to support the development of fusion power.

At this stage of development fusion power plants are largely conceptual. Research is needed to validate methods to predict system performance in the normal operating range and under potential accident conditions. The performance data are required to provide a basis for design. The same type of validated methods will also be required to quantitatively describe the environmental effects of operating fusion plants.

Based upon the information available on the system designs and the qualitative environmental analysis conducted in the previous section, the authors have identified some areas where R\&D is needed. During this effort, consideration was also given to the information necessary to predict the safety of the operating and maintenance personnel within fusion power plants.

The logic used to aid this R\&D identification is shown in Figure 2. The diagram is useful in illustrating the scenarios and system interactions that could lead to the accidental releases of toxic materials to the environmental, or lead to occupational safety problems or both. Each box in this diagram represents analyses that must be made or data that must be available to supply information for succeeding steps in the assessments.

Currently, the precise range of operating and transient parameters and the relative importance of the potential accidental pollutants are not well known. For these reasons, no attempt was made to suggest priorities for the R\&D. Further, it is not suggested that the R\&D recommended here are complete enough to provide a full understanding of facility safety when the plants reach operating status. Planning should continue to assure that relevant safety issues are being solved in a timely manner. The methods used here to initially identify R\&D needs can serve as a basis for a continuing effort.

Recommendations aimed at the timely development of relevant safety related information on fusion systems follow. General recommendations are made on how to continue the R\&D planning and identify priorities. Specific recommendations are also made for information and methods required to support reactor system design studies, operating performance predictions, and quantitative safety analyses. No effort has been made to determine whether the recommended information is available from other technology development programs, either within the U.S. or abroad. 
General Recommendation

A continuing pianning effort should be conducted to identify the safety related information needs in a priority consistent with the magnitude of the safety item, the potential uncertainty of the estimated effects based on existing technology, the time required to develop the information, and the date when this information is needed.

Specific Recommendations

The safety research recomended in this section is aimed at developing information such that fusion power reactors can be designed to effectively control potential plant accidents. These recommendations are found in Table 13. Additionally, research recomendations found in Table 14 are made to support design in the area of operational safety. The bases for these recommendations and other supporting information are found in Appendix 0 . 
IABLE 13. Recommendations for Safety Research and Development Relative to Accidents

\section{Analytical Area}

1. Primary Boundary Failure

2. Abnomal Plasma Transients

\section{Safety Concer}

Release of activation products and liquid metal to containment building

Plasma overpower, or local contact, with the first wall, may result in permanent damage or failure of the primary boundary Loss of magnet cooling resulting in gross structural failure

Release of low temperature coolant which can fail primary boundary and/or over pressurize the containment building.

Entrapment of solid air in insulation resulting in loss of insulation effective ness or structural damage upon warming to room temperature.

Formation of solid air in the cooling system can result in explosive mixtures of hydrogen and oxygen as burning of compounds which would otherwise not ignite when system is warmed to room temperature.

\section{Recommended $\mathrm{R} \&$}

Identify potential failure mechanisms and develop the corresponding failure criteria for the first wall, primary coolant system components and blanket structural materials. Consideration must be given to the operating parameters encountered during abnormal plan transients and nornial operation as well as the synergistic effects of various degradation processes.

Identify conditions for severe overpower and other plasma instabilities and determine the resulting energy spectra at the first wall.

Examine previous experimental devices to determine the cause and behavior of plasma instabilities that result in wall contact.

Develop simulation of cooling system and controls.

Develop simulation of air leakage into helium and insulation spaces.

Experinentally determine degradation of insulation due to vapor pressure increases. Develop physical properties at low tempera tures of potentially vulnerable structural material. 
TABLE 13. (Contd)

4. Contaimment Analysis

5. Abnormal Prinlary Flow Transients
Release of activation products to the public

Temperature changes due to loss of blanket flow or thermal ballast could cause failure of the primary boundary Large distortion of the magnet structure
could cause coolant blockages resulting in magnet quench and the rapid release of large stored energy.

Irradiation of organic insulators at cryogenic temperatures may result in stored energies which would be explosively re-
Develop properties of composite, stressed elements of containment systems.

Extend analytical methods which will

reliably predict the behavior of large size containment systems especially during pres-

sure reversals.

Develop simulation of primary coolant system and controls.

Determine effects of changing magnetic

fields on liquid metal flow and heat

transfer.

Determine thermal ballast requirements for pulsed systems

Develop digital computer structural codes which will simplify the analysis of highly heterogeneous structures.

Irradiation of polymers at cryogenic temperatures to determine stored energy and release upon warming. 
TABLE 14. Recommendations for Research and Development for Operational Safety

Analytical Area

1. Primary Boundary Leakage

2. Instrumentation Evaluation

Operational and Remote Maintenance Analys is

\section{Safety Concern}

Prediction of leakage of activation products and liquid metals to the containnent building will be necessary for establishing the adequacy of the effluent control systen and as sessing the maintainability and operability of the plant.

Adequate detection and location of leaks will be necessary to maintaining design limits on plant effluents and ensuring operationa

Adequacy of planic control and protection equipment in fusion power plant environment must be established to ensure plant malfunctions are properly identified.

Adequate personnel dosimetry does not currently exist to measure employee exposure from ionizing radiation from high energy sources.

Ensure that specified operation and ma intenance tasks can be carried out within established guidelines for employee materials present in a fusion power plant.

\section{Recoumended R \&}

See Radioactive Material Inventory and location for required $R \& D$.

Develop acoustic emission sensors (or acoustic signature) capable of operating in high temperature environs.

Develop a demonstration model of computer analyzer and acoustic emission sensors to evaluate applicability to preservice, inservice and continuous monitoring for leaks.

Develop detectors and monitors for high energy photons and neutrons including direct reading dosinetry.

Determine the effects of large e/m fields on dosimetry techniques.

Determine charged particle cross section data for dose equivalency for particles of greater than 3 Mev energy.

Develop remote maintenance and repair equipment and/or procedures.

Determine effects of e/in fields on plant personnel and public.

Develop test facility to conduct integrated remote welding and nondestructive test and inspection programs. 
TABLE 14. (Contd)

4. Radiodctive Material Inventoiv and Location

- First Wall Release of Radioactive Isotopes

\section{- Corrosion Product Activity} Control

- Iritium llandling and Storage
The concentration of radioactive material within all components of the primary

woundary becomponents of the primary

boundary becom source terms for assessing

the effects of both

Characterization of radioactive material in vacuum system is necessary for design and assessing the effects of system leakage and failure.

Minimize radioactive material concentration in primary coolant.

Tritium release from plant components to building containment and/or environs.
Determine sputtering coefficients and tritium hold-up of first wall materials.

Develop methodology for estimating corrosion Develop methodology for estimating corrosion product generation and activation in fusion
power plants. Develop conceptual systell for corrosion product conirol and removal.

Develop tritium permeation data for CTR operating environment.

Determine effect of corrosion on tritium permeation.

Define potential tritium losses during na in tenance.

Define tritium loss rates from components (valves, flanges, fittings).

Investigate adequacy of current tritium storage techniques to large inventories required in fusion power plants. 


\section{REFERENCES}

1. T. A. Coultas, J. E. Draley, V. A. Maroni and R. A. Krakowski, An Engineering Design of a Reference Theta-Pinch Reactor (RTPR), ANL-8019/LA-5336, Vo1. I., January 1974, Vol. II., March 1975.

2. R. W. Werner, G. A. Carlson, J. Hovingh, J. D. Lee, M. A. Peterson, Progress Report $\# 1$ on the Design Considerations for a Low Power Experimental Mirror Fusion Reactor, LLL Report, UCRL-74054, (1972).

3. R. W. Werner, G. A. Carlson, J. Hovingh, J. D. Lee, M. A. Peterson, Progress Report $\leqslant 2$ on the Design Considerations for a Low Power Experimental Mirror Fusion Reactor, LLL Report, UCRL-74054-2.

4. R. G. Mills, Ed., A Fusion Power Plant, Princeton University Plasma Physics Laboratory, MATT-1050, August 1974.

5. J. R. Powe11, Ed., "Preliminary Reference Design of a Fusion Reactor Exhibiting Very Low Residual Radioactivity," BNL-19565, Brookhaven National Lab., December 1974.

6. B. Badger, et a1., (30 authors), UWMAK-I, A Wisconsin Toroidal Fusion Reactor Design, UWFOM-68, University of Wisconsin, Vol. I (1974) and II (1975).

7. B. Badger, et a1., (30 authors), UWMAK-II, A Conceptual Tokamak Power Reactor Design, UWFDM-112, University of Wisconsin, October 1975 .

8. A. P. Fraas, Conceptual Design of the Blanket and Shield Region and Related Systems for a Ful1 Scale Toroidal Fusion Reactor, ORNL-TM-3096, May 1973.

9. P. G. Lorenzini and H. Pearlman, Environmental Assessment for UWMAK-I, Atomics International, Canoga Park, CA, April 1974:

10. United States Atomic Energy Commission (USAEC); Division of Controlled Nuclear Fusion, Fusion Power, An Assessment of U1timate Potential, (WASH-1239), February 1973.

11. J. Powell and W. C. Gough, "Intercomparison Among the Various Fusion Reactor Design Studies," in Fusion Reactor System Studies Review Meeting, (WASH-1278), USAEC, Germantown, MD, September 1973.

12. A. P. Fraas, A. Postma, Preliminary Appraisal of the Hazards Problems of a D-T Fusion Reactor Power Plant, (ORNL-TM-2822, Rev.) Oak Ridge National Laboratory, Oak Ridge, TN, December 1970.

13. F. N. Flakus, Fusion Power and the Environment, International Atomic Energy Agency, Vienna, Austria, October 1974.

14. D. Steiner and A. P. Fraas, "Preliminary Observations on the Radiological Implications of Fusion Power," Nuclear Safety, Vo1. 13, No. 5, September-October 1972.

15. A. P. Fraas, "Environmental Aspects of Fusion Power," presented at the Winter Annual Meeting of the American Nuclear Society, Washington, D.C., November 1972.

16. J. Kaser, Management of Nontritium Radioactive Wastes from Fusion Power Plants, BNWL-2019, Battelle, Pacific Northwest Laboratories, Richland, WA, July 1976.

17. B. Bennett, HASL, NY, "Environmenta? Tritium, The Dose to Man," Proceedings International Congress of International Radiation Protection Association (IRPA), Washington, O.C., September 1973.

18. J. W. Elwood, "Ecological Aspects of Tritium Behavior in the Environment," Nuclear Safety 12, 326-337, July-August 1971 .

19. P. J. Barry, Energy for Tomorrow, Nuclear Power and the Environment, AECL-5011, Chalk River Nuclear Laboratories, Chalk River, Ontario, December 1974. 
20. W. L. Marter, Environmental Effects of a Tritium Gas Release from the Savannah River Plant on May 24,1974, E. I. duPont DeNemours \& Co., Savannah River Laboratory, DP-1369, Aiken, SC.

21. A. A. Haghassi, M. W. Carter, Ed., "Health Physics Aspects of a Large Accidental Tritium Release," Tritium Symposium, August 28-September 3, 1971, Las Vegas, NV, Conference 710809 , May 1973.

22. D. G. Jacobs, Sources of Tritium and Its Behavior Upon Release to the Environment, TID 24635, NBS, U.S. Department of Comerce, Health Physics Division, Nuclear Safety Information Center, Oak Ridge National Laboratory, 1968.

23. 8. F. Gore and E. S. Murphy, Current Fusion Power Plant Design Concepts, BNWL-2013, Battelle, Pacific Northwest Laboratories, September 1976.

24. D. Steiner, "The Nuclear Performance of Fusion Reactor Blankets," Nuc.1. Appl. and Tech., g, 1970.

25. R. Bunde, et al., Aspects of Energy Supply by Fusion Reactors, (BNWL-TR-140), Translated from the German by Battelle, Pacific Northwest Laboratories, Richland, WA, April 1975 .

26. W. E. Kastenberg and D. Okrent, Safety and Environmental Effects of Central Station Fusion Power Reactors, UCLA, (To be published, Draft Juiy 1975).

27. D. K. Sze, "Emergency Cooling and Afterheat Effects of a CTR Blanket," The First Topical Meeting on the Technology of Controlled Nuclear Fusion, Vol. 1, p. 599, 1974.

28. T. E. Botts, W. L. Ferreli and A. Z. Ullman, Analysis of Loss of Flow Accidents in Lithium Cooled CTR Devices, UCLA, Department of Energy and Kinetics, Proceedings of the American Nuclear Society Winter Meeting, October-November 1975.

29. Recommendations of the International Commission on Radiological Protection, ICRP Pubiication 2, Report of Committee II on Permissibie Dose for Internal Radiation (1959), Pergamon Press, London, England, 1960.

30. D. L. Strenge, E. C. Watson and J. R. Houston, SUBDOSA - A Computer Program for Calculating External Doses from Accidental Atmospheric Releases of Radionuclides, BNWL-B-351, June 1975.

31. J. R. Houston, D. L. Strenge, E. C. Watson, DACRIN - A Computer Program for Calculating Organ Dose from Acute or Chronic Radionuclide Inhalation, BNWL-3-389, December 1974. 
The author acknowledges the timely contributions of $H$. Baumelberg, S. H. Bush, L. G. Faust,

A. B. Johnson, Jr., T. J. Kabele, I. 5. Levy, M. A. McKinnon, C. W. Stewart and M. Vagins. The critical reviews of the text by B. F. Gore, B. R. Leonard and others were particularly helpful.

The contributions of R. E. Rhoads in developing material for some of the appendixes and $D$. T. Aase for his analyses are especially noted. 
APPENOIXES 
APPENDIX A

HAZARDOUS MATERIAL

Table A-l is a compilation of chemically hazardous and radioactive substances known or implied to be present in the conceptual magnetic confinement designs. The table is divided according to the systems used in the model plant reference envelope and the inventory of each substance in that system is indicated where known. Various sources have been used in compiling this table. The majority of the information comes from the conceptual reactor design documents. Quantities from other sources are so indicated. Corrosion and activation products listed are from the waste treatment document. (1)

Compilation of this table is one of the first steps in a preliminary hazards analysis. It is only intended to list the materials which could be important in a detailed safety analysis. Potential release mechanisms were not considered. Nonradioactive substances are listed in the table if they are highly chemically reactive or if an Occupational Threshold Limit value has been set by the American Conference of Governmental Hygienists. Other materials which may prove hazardous, but for which no TLV exists are indicated at the end of the listing for each system. The table is necessarily incomplete and contains many estimates due to the lack of detailed design data.

\section{REFERENCES}

7. J. Kaser, Management of Nontritium Radioactive Wastes from Fusion Power Plants, 3NWL-2019, Battelle, Pacific Northwest Laboratories, Richland, WA, JuTy 1976. 
TABLE A-1. Compilation of Potentially Hazardous Materials

\begin{tabular}{|c|c|c|c|c|c|}
\hline Device & Material & Form, Use, Etc. & $\begin{array}{c}\text { Inventory } \\
(M T *)\end{array}$ & Radioactivity & $\begin{array}{c}T L V(d) \\
\left(m g / m^{3}\right) \\
\end{array}$ \\
\hline \multirow[t]{10}{*}{ UWMAX I } & Lf & Liquid, T2500 $\mathrm{C}$ & 1700 & Entrained $T$ & $(\mathrm{LiH}) .025^{(b)}$ \\
\hline & $\mathrm{T}$ & In blanket & $4 \mathrm{~kg},(c)$ & $3.9 \times 10^{7} \mathrm{Ci}$ & -. \\
\hline & $\tau$ & In blanket coolant & $3.7 \mathrm{~kg}$ & (0) & $-\cdot$ \\
\hline & Corrosion prod. & Cr Oxides & $.35 / y^{\prime r}(d)$ & (9) & 1 \\
\hline & & Mn Oxides & $.05 / \mathrm{yr}$ & & 5 \\
\hline & & Ni Oxides & $.45 / y r$ & $4 \times 10^{6} \mathrm{Ci} / \mathrm{yr}$ & 1 \\
\hline & & Mo Oxides & $.08 / y r$ & total & 15 \\
\hline & & Fe Oxides & $1.55 / \mathrm{yr}$ & & 10 \\
\hline & & Co Oxides & trace & & .1 \\
\hline & & V Oxides & trace & & .5 \\
\hline \multirow[t]{4}{*}{ BNL $(e)$} & $\mathrm{Be}$ & Metal spheres & 190 & Entrained $T$ & .002 \\
\hline & Graphite & -. & 3000 & $c^{14}$ & 15 mppcf $(e)$ \\
\hline & $\tau$ & In blanket & 149 & $9721 \mathrm{C} / / \mathrm{gm}$ & -- \\
\hline & T & In blanket coolant & $\mathrm{lg}$ & $9721 \mathrm{Ci} / \mathrm{gm}$ & -- \\
\hline \multirow[t]{10}{*}{ PPPL Tokamak } & Be & In Five & $375^{(9)}$ & & .002 \\
\hline & LiH & Magnet shield & & -- & .025 \\
\hline & Corrosion prod. ${ }^{(h)}$ & Wi Oxides, fluorides & $.3 / \mathrm{yr}$ & & 1 \\
\hline & & Fe Oxides, fluorides & $.3 / y r$ & $3.6 \times 10^{7} \mathrm{Ci} / \mathrm{yr}$ & 10 \\
\hline & & Cr Oxides, fluorides & $12 / y r$ & total & 1 \\
\hline & & Co Oxides, fluorides & trace & & .1 \\
\hline & & $\checkmark$ oxides, fluorides & trace & & .5 \\
\hline & T & In blanket & $10 \mathrm{~g}$ & $10^{5} \mathrm{Ci}$ & .. \\
\hline & $T$ & In blanket coolant & $10 \mathrm{~g}$ & $10^{5} \mathrm{Ci}$ & -. \\
\hline & Corrosion prod. ${ }^{(i)}$ & In He coolant & $\begin{array}{l}10 \mathrm{gm} / \mathrm{yr} \\
\text { total }\end{array}$ & $40 \mathrm{Ci} / \mathrm{yr}$ & $\ldots$ \\
\hline \multirow[t]{5}{*}{ ORNL Tokamak } & Li & Liquid, Tr $1050^{\circ} \mathrm{C}$ & 462 & Entrained $T$ & $(\mathrm{LiH}) .025^{(\mathrm{y})}$ \\
\hline & Graphite & -- & $1100(j)$ & $c^{14}$ & 15 mppcg \\
\hline & Corrosion prod. & Wh Oxides & $1.3 / \mathrm{yr}$ & $4 \times 10^{6} \mathrm{Ci} / \mathrm{yr}$ & -. \\
\hline & & Zr Oxides & $.01 / \mathrm{yr}$ & total & 5 \\
\hline & $T$ & In blanket coolant & $120 \mathrm{~g}$ & $1.2 \times 10^{5} \mathrm{Ci}$ & -- \\
\hline \multirow[t]{7}{*}{ S-Pinen } & Li & 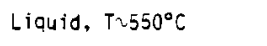 & 860 & Entrained $T$ & (LiH) $.025^{(b)}$ \\
\hline & $\mathrm{Be}$ & Metal & วิ0 & -- & .002 \\
\hline & Graphi te & -- & 680 & $c^{14}$ & 15 mppef \\
\hline & Corrosion prod. & Nb Oxides & $2 / y r$ & $10^{7} \mathrm{Ci} / \mathrm{yr}$ & 5 \\
\hline & & Zr Oxides & $.02 / y r$ & & -- \\
\hline & $\mathrm{T}^{\mathrm{ccc}}$ & In blanket & 539 & $5.2 \times 10^{5} \mathrm{Ci}$ & -- \\
\hline & & In blanket coolant & $404 \mathrm{~g}$ & $3.9 \times 10^{6} \mathrm{Ci}$ & -- \\
\hline \multirow[t]{11}{*}{ MIRRDR } & ii & Liquid & a3 & Entrained $T$ & $(L i H) .225$ \\
\hline & Graphite & .. & & $c^{14}$ & 15 mppef \\
\hline & Corrosion prod. & Fe oxides $(k)$ & & & 10 \\
\hline & & iti Oxides & & & 1 \\
\hline & & Cr Oxides & & ' & 1 \\
\hline & & Yo Oxides & & $\therefore 1.5 \mathrm{Ci} / \mathrm{gm}$ & 15 \\
\hline & & Yn Oxides & & & 5 \\
\hline & & So Oxides & trace & & .1 \\
\hline & & y oxides & trace & & .5 \\
\hline & T & in bianker & & & .. \\
\hline & $T$ & In olankec coolant & 〈kg. & {$\left[9.7 \times 10^{\circ}[i>\right.$} & $\cdot-$ \\
\hline
\end{tabular}


TABLE A-T. (Contd)

\begin{tabular}{|c|c|c|c|c|c|}
\hline Device & Material & Form, Use, etc. & $\begin{array}{c}\text { Inventory } \\
\left(M T^{*}\right) \\
\end{array}$ & Radioactivity & $\begin{array}{l}\text { TLV(d) } \\
\left(\mathrm{mg} / \mathrm{m}^{3}\right)\end{array}$ \\
\hline \multirow[t]{8}{*}{ UWMAK I } & $\mathrm{Na}$ & Liquid, $T^{\circ}-450^{\circ} \mathrm{C}$ & $1.8 \times 10^{4}$ & Entrained $T$ & $(\mathrm{NaOH}) 2^{(b)}$ \\
\hline & \multirow[t]{6}{*}{ Corrosion prod. } & in Oxides & $5.4 \mathrm{gm} / \mathrm{yr}$ & & 5 \\
\hline & & Cr oxides & $48.6 \mathrm{gm} / \mathrm{yr}$ & & 1 \\
\hline & & iti Oxides & $32.4 \mathrm{gm} / \mathrm{yr}$ & $400 \mathrm{ci} / \mathrm{yr}$ & 1 \\
\hline & & Fe Oxides & $180.9 \mathrm{gm} / \mathrm{yr}$ & & 10 \\
\hline & & Co Oxides & trace & & .1 \\
\hline & & $V$ oxides & trace & & .5 \\
\hline & $T$ & in ita coolant & $\therefore 9$ & $\therefore 10^{4} \mathrm{Ci}$ & -. \\
\hline $\begin{array}{l}\text { 3ill (reference } \\
\text { of text) }\end{array}$ & e 10 & In He coolant & & $\mathrm{g72I} \cdot \mathrm{Ci} / \mathrm{gm}$ & $-\cdot$ \\
\hline \multirow[t]{6}{*}{ PPPL T-1.amak } & \multirow[t]{5}{*}{ Corrosion prod. } & ili Oxides & $4.3 \mathrm{gm} / \mathrm{yr}$ & & 1 \\
\hline & & Fe Oxides & $3.9 \mathrm{gm} / \mathrm{yr}$ & & 10 \\
\hline & & Cr Oxides & $1.8 \mathrm{gm} / \mathrm{yr}$ & $40 \mathrm{Ct} / \mathrm{yr}$ & 1 \\
\hline & & Co Oxides & trace & & .1 \\
\hline & & $V$ oxides & trace & & .5 \\
\hline & $\mathrm{T}$ & In the coolant & & $9721 \mathrm{Ci} / \mathrm{gm}$ & -- \\
\hline \multirow[t]{4}{*}{ ORNL TOKAMAK } & $\mathrm{x}$ & Liquid, Ta980 $\mathrm{C}$ & 10 & Entrained $T$ & $(\mathrm{NaOH}) 2^{(b)}$ \\
\hline & \multirow[t]{2}{*}{ Corrosion prod. } & ito oxides & $20 \mathrm{gm} / \mathrm{yr}$ & & -- \\
\hline & & Zr oxides & $.2 \mathrm{gm} / \mathrm{yr}$ & $100 \mathrm{Cf} / \mathrm{yr}$ & 5 \\
\hline & $T$ & In $k$ coolant & & $9721 \mathrm{Ci} / \mathrm{gm}$ & -- \\
\hline \multirow[t]{5}{*}{ E-PINCH } & ila & Liquid, $T=500^{\circ} \mathrm{C}$ & not specified & Entrained $T$ & $(\mathrm{NaOH}) 2^{(\mathrm{b})}$ \\
\hline & \multirow{2}{*}{$\begin{array}{c}\text { ila } \\
\text { Corrosion prod. }\end{array}$} & Ho Oxides & & & -- \\
\hline & & Zr Oxides & & $5 \mathrm{Ci} / \mathrm{gm}$ & 5 \\
\hline & \multirow[t]{2}{*}{$T^{\{m\}}$} & In iva coolant & $0.3 g(c c)$ & $2.9 \times 10^{3} \mathrm{Ci}$ &.- \\
\hline & & In Steam & $\therefore 9$ & $6.8 \times 10^{4} \mathrm{Ci}$ & -- \\
\hline \multirow[t]{8}{*}{ MIRROR } & \multirow[t]{7}{*}{ Corrosion prod. $(k)$} & Fe Oxides & (f) & & 10 \\
\hline & & Ni Oxides & & & 1 \\
\hline & & Cr Oxides & & & 1 \\
\hline & & Yo Oxides & & $1.5 \mathrm{ci} / \mathrm{gm}$ & 15 \\
\hline & & inn Oxices & & & 5 \\
\hline & & Co Oxides & trace & & .1 \\
\hline & & Oxides & trace & & .5 \\
\hline & $\mathrm{T}$ & In the coolant & & $9721 \mathrm{Ci} / \mathrm{gm}$ & $\cdot$. \\
\hline
\end{tabular}


TABLE A-1. (Contd)

\begin{tabular}{|c|c|c|c|c|c|}
\hline Jevice & Material & Form, Use, etc. & $\begin{array}{c}\text { inventory } \\
(M \top *)\end{array}$ & Radioactivity & $\begin{array}{r}T L V(d) \\
\left(\mathrm{mg} / \mathrm{m}^{3}\right) \\
\end{array}$ \\
\hline \multirow[t]{14}{*}{ UWMAK ! } & Li & Liquid, $T r 325^{\circ} \mathrm{C}$ & & Entrained $\mathrm{T}$ & $(\mathrm{LiH}) .025^{(b)}$ \\
\hline & $\mathrm{Na}$ & Heat removal & & Entrained $T$ & $(\mathrm{NaOH}) 2^{(b)}$ \\
\hline & $T$ & Trapped in Li & $3.5 \times 9$ & $3.5 \times 10^{7}$ & -. \\
\hline & $T$ & In vacuum 5 ys tem & $0.3 \mathrm{~kg}$ & $3 \times 10^{6}$ &.- \\
\hline & Air Activation & ${ }^{41} \mathrm{Ar}$ & 5 -gm & $200 \mathrm{Ci}$ & - \\
\hline & prod. (o) & $13 \mathrm{~N}$ & $3 \mathrm{mg}$ & $4 \times 10^{6} \mathrm{Ci}$ & -. \\
\hline & & ${ }^{14} \mathrm{C}\left(\right.$ as $\mathrm{CO}_{2}$ ) & $18 \mathrm{mg}$ & $.08 \mathrm{Ci}$ & $\cdots$ \\
\hline & Corrosion prod. $(p)$ & Cr Oxides & $.22 / \mathrm{yr}$ & & 1 \\
\hline & & Mn Oxides & $.03 / \mathrm{yr}$ & & 5 \\
\hline & & Mi Oxides & $.29 / \mathrm{yr}$ & & 1 \\
\hline & & Mo Oxides & $.05 / \mathrm{yr}$ & $2.4 \times 10^{6} \mathrm{Ci} / \mathrm{yr}$ & 15 \\
\hline & & Fe Oxides & $1.01 / y r$ & & 10 \\
\hline & & Co Oxides & trace & & .1 \\
\hline & & V Oxides & trace & & .5 \\
\hline \multirow[t]{4}{*}{ BNL } & $\mathrm{T}$ & in vacuum system & & $9721 \mathrm{Ci} / \mathrm{gm}$ & $-\cdot$ \\
\hline & Air activation prod. & ${ }^{41} \mathrm{Ar}$ & & $42 \mathrm{Ci} / \mathrm{Lgm}$ & $\cdots$ \\
\hline & & ${ }^{13} \mathrm{~N}$ & & $1700 \mathrm{ci} / \mathrm{hgm}$ & $-\cdot$ \\
\hline & & ${ }^{14} \mathrm{C}\left(\mathrm{as} \mathrm{CO}_{2}\right)$ & & $4.3 \mathrm{Ci} / \mathrm{gm}$ & - \\
\hline \multirow[t]{9}{*}{ PPPL TOKAMAK } & $\mathrm{T}$ & In vacuum system & & & -- \\
\hline & Corrosion prod. & Ni Oxides & $.86 / \mathrm{yr}$ & & 1 \\
\hline & & Fe Oxides & $.78 / \mathrm{yr}$ & & 10 \\
\hline & & $\mathrm{Cr}$ oxides & $.36 / \mathrm{yr}$ & $7 \times 10^{5} \mathrm{Cj} / \mathrm{yr}$ & 1 \\
\hline & & Co Oxides & trace & & .1 \\
\hline & & y oxides & trace & & .5 \\
\hline & Air activation prod. & ${ }^{41} \mathrm{Ar}$ & $5: \mathrm{gm}$ & $200 \mathrm{Ci}$ & -- \\
\hline & & $13 \mathrm{~N}$ & $3 \mathrm{mg}$ & $4 \times 10^{6} \mathrm{Ci}$ & - \\
\hline & & ${ }^{14} \mathrm{C}$ (as $\mathrm{CO}_{2}$ ) & $18 \pi g$ & $.08 \mathrm{Ci}$ & -- \\
\hline \multirow[t]{3}{*}{ ORNL TOKAMAK ${ }^{10}$} & a) & [n vacuum system & $0.5 \mathrm{~kg}$ & $5 \times 10^{6} \mathrm{Ci}$ & -. \\
\hline & Corrosion prod. & Nb Oxides. & $240 \mathrm{~kg} / \mathrm{yr}$ ? & $1.4 \times 10^{6} \mathrm{Ci}$ & 5 \\
\hline & & Zr Oxides & $2 \mathrm{~kg} / \mathrm{yr}\}$ & & -- \\
\hline 2-PINCH $(r)$ & T & (dd) & $i .6 \mathrm{~kg}$ & $1.6 \times 10^{7}$ & $\cdots$ \\
\hline \multirow[t]{11}{*}{ :IIRROR } & $T$ & In vacuum system & & & -- \\
\hline & Corrosion prod. $(\mathrm{s})$ & Cr oxides & 1 & & 1 \\
\hline & & Mn Oxides & & & 5 \\
\hline & & Ni Oxides & & & 1 \\
\hline & & Mo Oxides & & $1.5 \mathrm{ci} / \mathrm{gm}$ & 15 \\
\hline & & Fe Oxides & & & 10 \\
\hline & & Co Dxides & trace & & .1 \\
\hline & & Oxides & trace & & .5 \\
\hline & Air activation prod. & $42 r$ & & $42 C i /-g m$ & $\cdots$ \\
\hline & & $13 y$ & & $1700 \mathrm{Ci} / \mathrm{gm}$ & -- \\
\hline & & ${ }^{i t}=\left(a s: \mathrm{O}_{2}\right\}$ & & $4.3 \mathrm{ci} / \mathrm{gm}$ & - \\
\hline \multicolumn{6}{|c|}{ Other materials: } \\
\hline
\end{tabular}


TABLE A-1. (Contd)

\begin{tabular}{|c|c|c|c|c|c|}
\hline Device & Material & Form, Use, etc. & $\begin{array}{l}\text { Inventory } \\
\left.(M)^{*}\right)\end{array}$ & Radioactivity & $\begin{array}{l}\text { iLvta) } \\
\left(\mathrm{mg} / \mathrm{m}^{3}\right) \\
\end{array}$ \\
\hline \multirow[t]{13}{*}{ UWMAK I } & Li & Liquid, $T \cdot 360^{\circ} \mathrm{C}$ & $14.4^{(u)}$ & Entrained $T$ & $(\mathrm{LiH}) .02^{(b)}$ \\
\hline & $\mathrm{Na}$ & Liquid, $\mathrm{T} 340^{\circ} \mathrm{C}$ & $1.8 \times 10^{3}(u)$ & Entrained $T$ & $(\mathrm{NaOH}) 2^{(b)}$ \\
\hline & Corrosion prod. & Cr Oxides & $14 \mathrm{gm} / \mathrm{yr}$ & 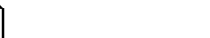 & 1 \\
\hline & & Mn Oxides & $2 \mathrm{gm} / \mathrm{yr}$ & & 5 \\
\hline & & Ni Oxides & $18 \mathrm{gm} / \mathrm{yr}$ & & 1 \\
\hline & & Mo Oxides & $3 \mathrm{gm} / \mathrm{yr}$ & $150 \mathrm{Ci} / \mathrm{yr}$ & 15 \\
\hline & & Fe Oxides & $62 \mathrm{gm} / \mathrm{yr}$ & & 10 \\
\hline & & Co Oxides & trace & & .1 \\
\hline & & $\checkmark$ Oxides & trace & & .5 \\
\hline & Air activation & 41 Ar & 5 gm & $200 \mathrm{Ci}$ & -- \\
\hline & $\operatorname{prod} .(v)$ & $13 \mathrm{~N}$ & $3 \mathrm{mg}$ & $4 \times 10^{5} \mathrm{Ci}$ & -. \\
\hline & & ${ }^{14} \mathrm{C}$ (as $\mathrm{CO}_{2}$ ) & $18 \mathrm{mg}$ & $.08 \mathrm{Ci}$ & $\cdots$ \\
\hline & $T$ & Yttrium Bed & $1.0 \mathrm{~kg}(\mathrm{w})$ & $9.9 \times 10^{6} \mathrm{Ci}$ & $\cdots$ \\
\hline \multirow[t]{5}{*}{ BNL } & Corrosion prod. & Al Oxides & (f) & 2.3 min half-tife & -- \\
\hline & Air activation & $4 i_{\mathrm{Ar}}$ & & $42 \mathrm{Ci} / \mathrm{Lgm}$ & -- \\
\hline & prod. (V) & $13 y$ & & $1700 \mathrm{Ci} / \mathrm{gm}$ & $\cdots$ \\
\hline & & ${ }^{14} \mathrm{C}\left(\mathrm{As}_{5} \mathrm{CO}_{2}\right)$ & & $4.3 \mathrm{Ci} / \mathrm{gm}$ & .. \\
\hline & $T$ & \multicolumn{3}{|c|}{ not specified } & $\cdots$ \\
\hline \multirow[t]{10}{*}{ PPPL TOKAMAK } & Be & In flibe & & -- & .002 \\
\hline & Corrosion prod. & Ni Oxides & $\left..3 / y r^{(x)}\right]$ & & 1 \\
\hline & & Fe Oxides & $.3 / y r$ & & 10 \\
\hline & & Cr Oxides & $12 / \mathrm{yr}$ & $3.6 \times 10^{7} \mathrm{Cj} / \mathrm{yr}$ & 1 \\
\hline & & Co Oxides & trace & & .1 \\
\hline & & $Y$ Oxides & trace & & .5 \\
\hline & Air activation & $4 I_{\mathrm{Ar}}$ & 5 L L & $200 \mathrm{Ci}$ & -- \\
\hline & prod. (v) & $13 i$ & $3 \mathrm{mg}$ & $4 \times 10^{6} \mathrm{Cl}$ & $\cdots$ \\
\hline & & ${ }^{14} \mathrm{C}$ (as $\mathrm{CO}_{2}$ ) & $18 \mathrm{mg}$ & $.08 \mathrm{Ci}$ & -- \\
\hline & $T$ & & not specified & . & \\
\hline \multirow[t]{4}{*}{ ORNL TOKAMAK } & $T$ & ' & & & \\
\hline & Corrosion prod. & No Oxides & $200 \mathrm{gm} / \mathrm{yr}$ \} & & 5 \\
\hline & & Zr Oxides & $2 \mathrm{gm} / \mathrm{yr}\}$ & $1000 \mathrm{Cj} / \mathrm{yr}$ & -- \\
\hline & $k$ & Liquid, T,930 $0^{\circ} \mathrm{C}$ & $2^{(4)}$ & Entrained : & (b) \\
\hline \multirow[t]{4}{*}{ J-PINCH } & $\top$ & & 2709 & $\cdot$ & $\cdots$ \\
\hline & Corrosion prod. & No Oxides & & $.5 \mathrm{cj} / \mathrm{gm}$ & -- \\
\hline & & Ir Oxides & & & \\
\hline & $\operatorname{Li}$ & Liquid, Tw $410^{\circ} \mathrm{C}$ & & Entrained $T$ & $(\mathrm{LiH}) .025^{(b)}$ \\
\hline \multirow[t]{11}{*}{ MIRROR } & $T$ & & not specified & & \\
\hline & Air activation & ${ }^{41}$ Ar & & $\$ 2[i / 2 g m$ & $-\cdot$ \\
\hline & prod. $(v)$ & $\sqrt{3} 4$ & & $1700 \mathrm{ci} / \mathrm{Lgm}$ & -- \\
\hline & & ${ }^{14} \mathrm{C}$ (as $\left.\mathrm{CO}_{2}\right)$ & & $4.3 \mathrm{Cl} / \mathrm{gm}$ & -- \\
\hline & Corrosion prod. ${ }^{(k)}$ & Fe Oxides & $(f)$ & & 10 \\
\hline & & Cr Oxides & & & 1 \\
\hline & & Ni Oxides & & & 1 \\
\hline & & Mo Oxides & & $1.5 \mathrm{ci} / \mathrm{gm}$ & 15 \\
\hline & & Mn Dxides & & & 5 \\
\hline & & Co Oxides & trace & & .1 \\
\hline & & yoxiges & trace & & .5 \\
\hline \multirow[t]{3}{*}{ Other materials: } & 1) Yttrium foil & & & & \\
\hline & 2) LiC? & & & & \\
\hline & 3) KCl & & & & \\
\hline
\end{tabular}


TABLE A-1. (Contd)

\section{E. Plasma Heating System:}

All devices except the G-pinch heat the plasma at least partially with some form of particle injection system. This system will contain $T$, and the components which are contained inside the blanket region will become activated.

\section{F. Electricity Generation System:}

Some small amounts of $T$ may be expected to diffuse into the steam system.

G. Confinement System:

$\mathrm{NbTi}$ and $\mathrm{Nb}_{3} \mathrm{Sn}$ superconducting material are used in large quantities. The toxicity of these compounds is not well defined.

H. Fuel System:

A supply of $T$ will have to be stored on site. Amounts are not specified. It is expected to be in the $\mathrm{kg}$ range for most devices (e.g., $1.4 \mathrm{~kg}$ for the 2 -pinch [reference 1 of text]). 
a. Threshold Limit Value in air for occupational exposure as set by American Conference of Governmental Hygenists. These values are for nonradioactive substances only.

b. See Appendix $C$ for discussion of alkali metal reaction products.

c. Values in $<$ come from reference 11 in text or are derived from information presented there.

d. Inventories of corrosion products are taken from the Waste Treatment Section of this paper. They are available only in quantity removed/yr. Actual inventories at any one time will be some unknown fraction of this quantity. Quantities of individual elements are based on equal corrosion rates and estimated weight percentage of that element in the parent substance.

e. Million parts per cubic foot of air.

f. Amount of corrosion products in He-cooled devices should be much smaller than in liquid metal cooled devices. They should be essentially limited to products that are neutron sputtered from the coolant tube walls and should be less than $10 \%$ of the amounts corroded by liquid metals.

g. Quantities in brackets are from reference 10 in text.

h. In $f l$ ibe and flibe cleanup system.

i. Constituents and TLV's same as those in flibe system.

j. 3000 metric tons more in magnet shield

k. The type of stainless steel used is not specified. Type 316 assumed here.

1. The BNL design deals only with the blanket. The materials listed for other systems are inferred from the blanket design.

m. Construction of intermediate heat exchangers limits the permeation of $T$ into the $\mathrm{Na}$.

n. Includes Vacuum System.

o. quantities and activities listed for activation products from air which leaks into the plasma region are equilibrium values assuming continuous operation. The amount present in the spent plasma collection system at any one time will vary from this.

p. Corrosion products from first wall sputtering/erosion. Amounts based on estimated erosion rates and equal erosion of all the constituents of the first wall.

q. The ORNL Tokamak design includes a vacuum system surrounding the plasma region. Air inleakage and resultant activation in the plasma region should be small.

$r$. The theta-pinch design includes a layer of neutral fuel atoms around the plasma. Air inleakage and activation should therefore be small.

s. First wall material is specified only as stainless steel. Type 316 is assumed here.

t. Includes gas separation system. 
u. Estimates based on percentage of total flow through this system.

v. In gas separation system. Quantities and activities presented are equilibrium values for the whole plant assuming constant operation. The amount actually present in the gas separation system will vary from this.

w. Reference 9 in text.

$x$. Assumes all corrosion products in flibe flow through this system.

y. Based on breeding ratio of 1.04 and daily consumption of $0.5 \mathrm{~kg}$.

z. Based on breeding ratio of 1.52 and daily consumption of $0.12 \mathrm{~kg}$.

aa. Based on breeding ratio of 1.18 and daily consumption of $1.5 \mathrm{~kg}$.

bb. Based on breeding ratio of 1.37 and daily consumption of $80 \mathrm{gm}$.

cc. Reference 1 of text.

dd. In spent plasma collection, tritium extraction and fuel injection system (reference 1 of text). 
APPENDIX B

\section{STORED ENERGIES}

Table B-1 lists the stored energies in the various conceptual fusion designs. In some cases, the designs lack sufficient detail to calculate the magnitudes of these stored energies. Many of the numerical values listed are estimates, intended to serve only as guides for the order of magnitude of the particular item. All sources of stored energy that could presently be identified have been listed without evaluation of the importance of the individual items.

This compilation is intended to give some perspective on the different stored energies in fusion power plants with magnetically confined plasmas. The compilation is also an initial step in a preliminary hazards analysis where only the magnitudes of the stored energies are noted. Equally important in accident analysis and safety reviews but not considered here because of the conceptual nature of the designs, are release mechanisms and rates of release of the stored energies. 


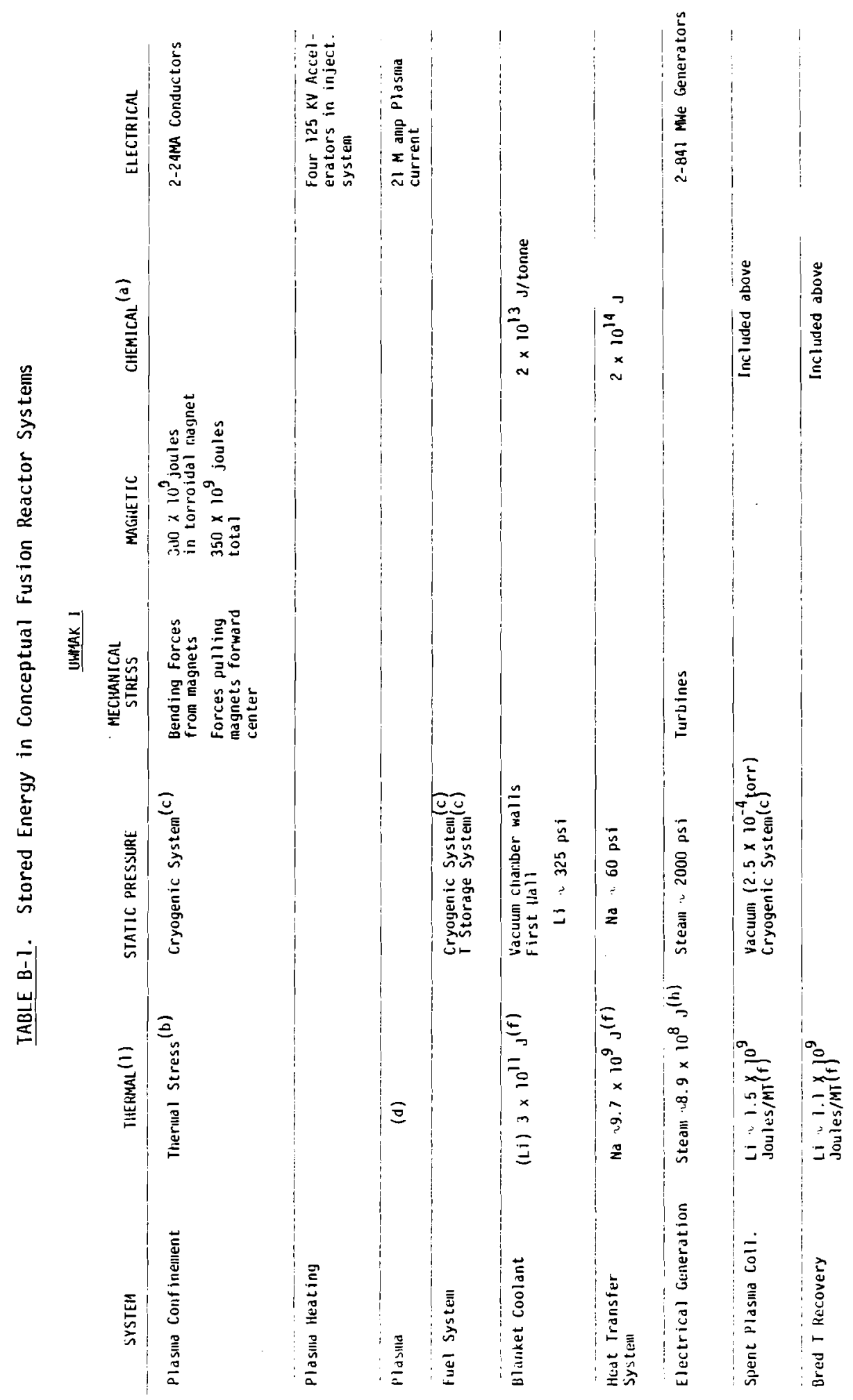

B. 2 
TABLE B-1. (Contd)

BNI

\begin{tabular}{|c|c|c|c|c|c|c|}
\hline SYSTEM & IIIERMAL & & STATIC PRESSURE & IIECHAAIICAL STRLSS & MAGHE UIC & ELECTRICAI. \\
\hline Plasma Conf inement & & Not Described & & & & \\
\hline Plasila lleating & & Not Described & but are same as UWMAK I & & & \\
\hline Pla stllia & & Not Described & & & & \\
\hline Fuel Systemin & & Not Described & & & & \\
\hline Blanket Coolant & He $4.5 \times 1$ & $10^{10} J^{(h)}$ & $\begin{array}{l}\text { Vacuum Chamber Walls } \\
\text { first Wall } \\
\text { lle } 450 \text { psi }\end{array}$ & & & \\
\hline Hedt Iransfer & He $4.5 \times 10$ & $10^{10} \mathrm{~J}^{(\mathrm{h})}$ & ite $450 \mathrm{psi}$ & & & \\
\hline $\begin{array}{l}\text { Electrical Geners- } \\
\text { Lion }\end{array}$ & $\begin{array}{l}\text { Steam } 24.6 \\
1.1 \times 10^{9}\end{array}$ & $\begin{array}{l}\times 10^{6} \text { joules } / \mathrm{kg}^{(h)} \\
\mathrm{J}(\mathrm{h})\end{array}$ & Steam 2400 psi & Turbines & & $\begin{array}{c}\text { Generators - } 1208 \mathrm{MWE} \\
\text { and } 511 \mathrm{MWE}\end{array}$ \\
\hline Suent Plasila Call. & $\begin{array}{l}\text { In coolant } \\
\text { specified) }\end{array}$ & (amount not & $\begin{array}{l}\text { Vacuum } \\
\text { Cryogenic System }(c)\end{array}$ & & & \\
\hline 8red I Recovery & He $1.0 \times 1$ & $10^{6}$ joules $/ \mathrm{Kg}_{9}(\mathrm{~h})$ & $\begin{array}{l}\text { Vacuum } \\
\text { Cryogen ic System( }\end{array}$ & & & \\
\hline
\end{tabular}




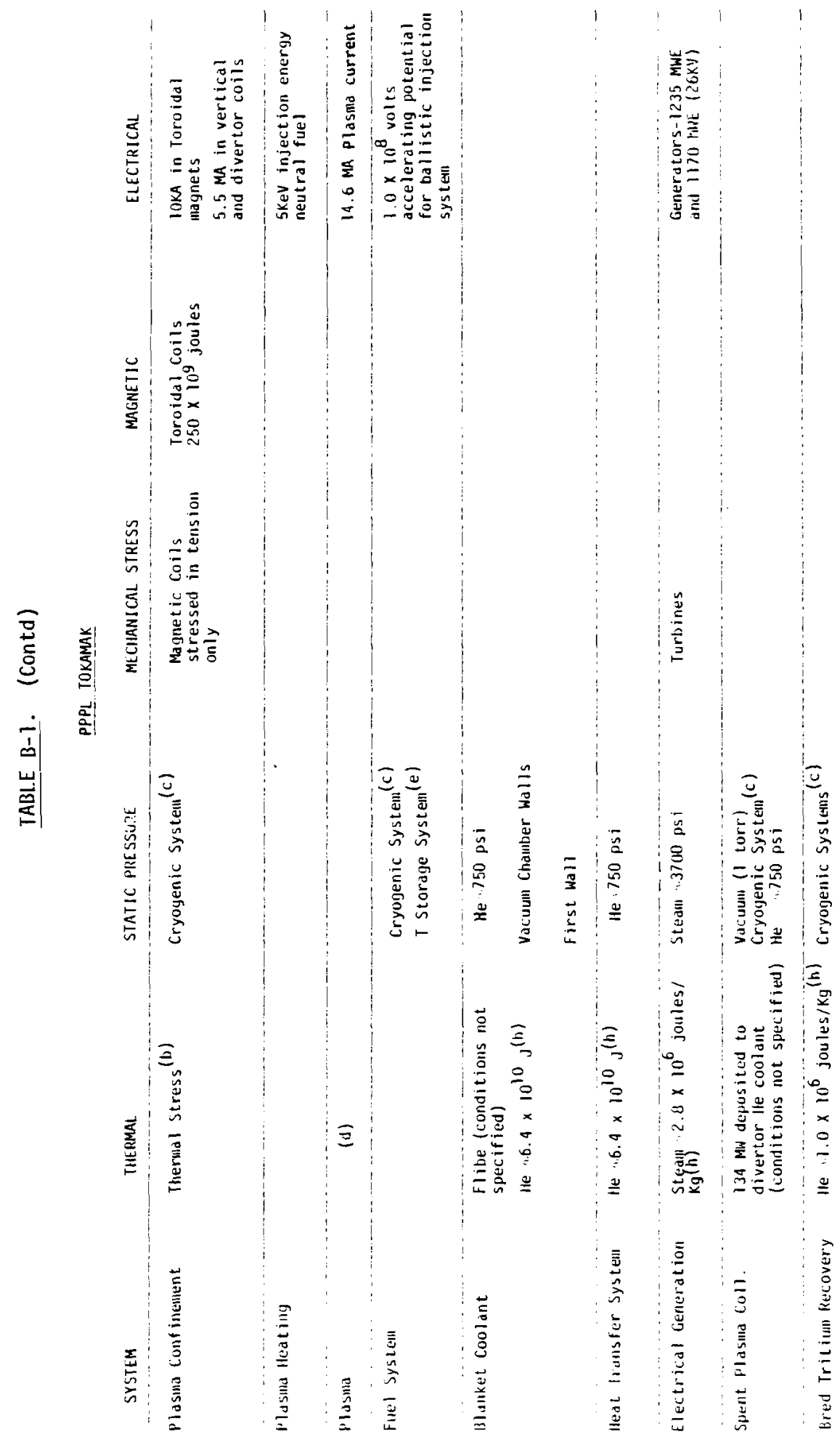




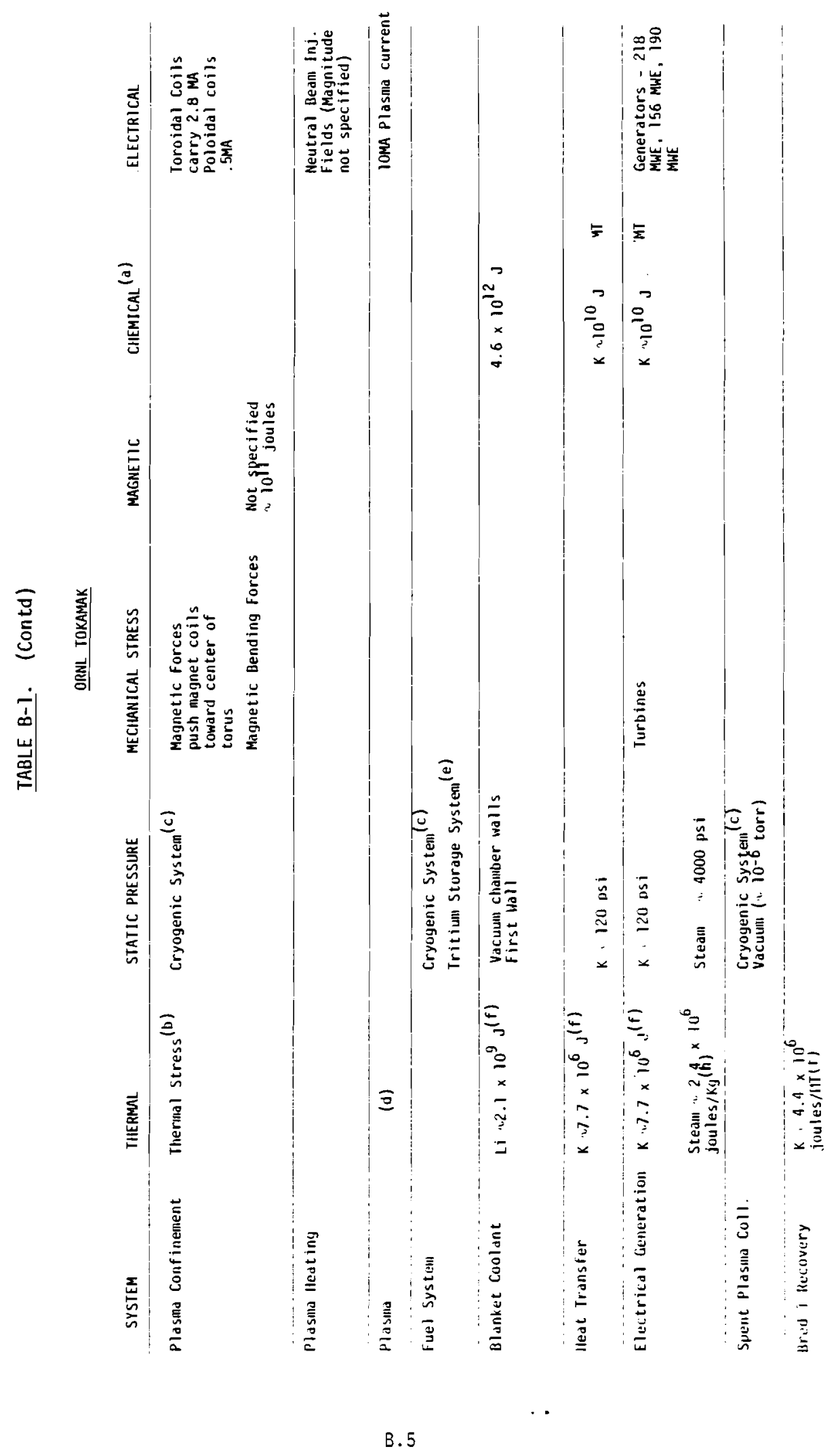




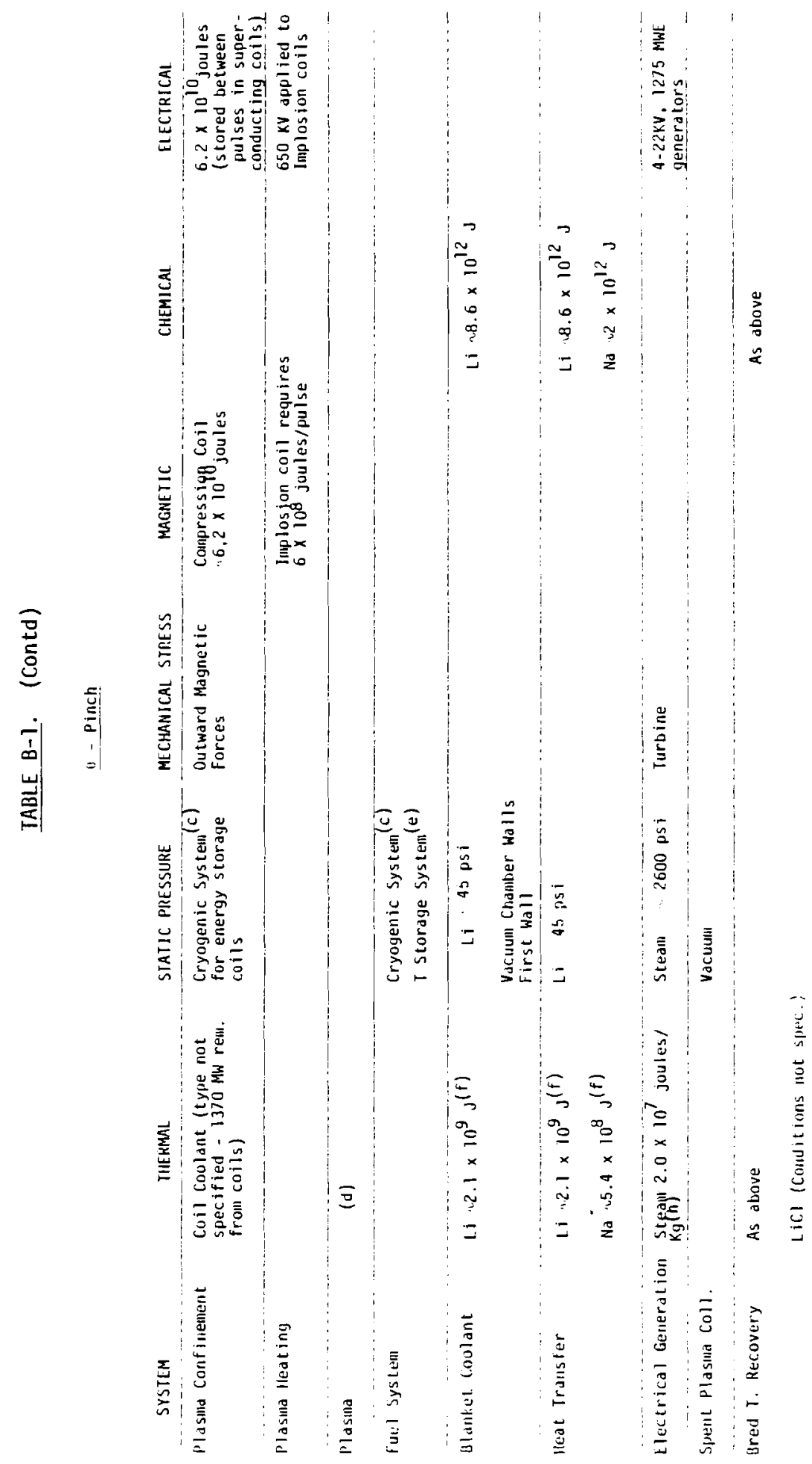




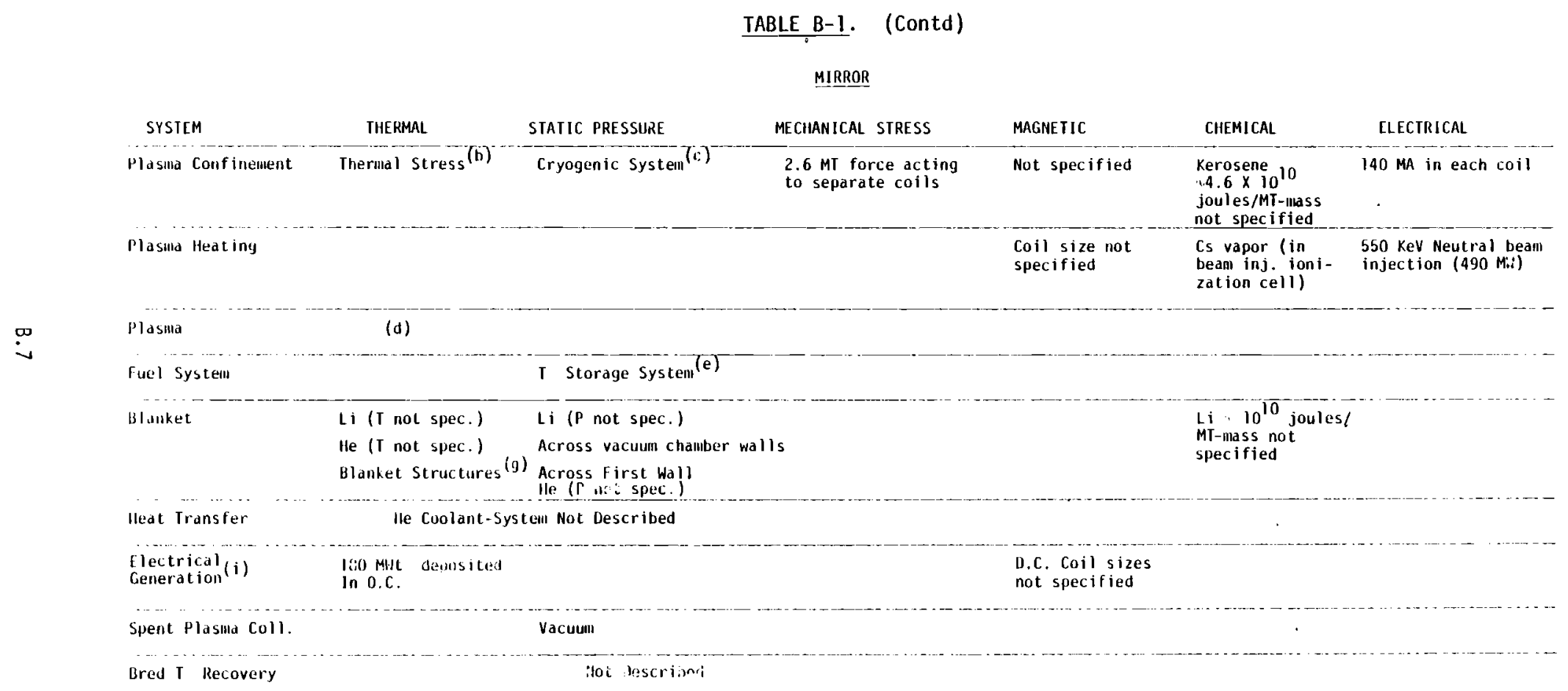


NOTES FOR TABLE B-1

a) Chemical potentials of the alkali metals are based on the data (2) in the table below. Complete combustion of 1 MT of the metal was assumed in calculating the heat generated.

\section{Reaction}

$2 \mathrm{M}+\mathrm{H}_{2} \mathrm{O}+\mathrm{M}_{2} \mathrm{O}+\mathrm{H}_{2}$ $2 \mathrm{M}+1 / 2 \mathrm{O}_{2}+\mathrm{M}_{2} \mathrm{O}$
Energy Released (Joules/MT)

$\begin{array}{cll}\frac{\mathrm{Li}}{2.46 \times 10^{10}} \frac{\mathrm{Na}}{3.68 \times 10^{9}} & \frac{\mathrm{K}}{1.47 \times 10^{9}} \\ 4.18 \times 10^{10} & 8.9 \times 10^{9} & 4.6 \times 10^{9}\end{array}$

b) There will be large thermal gradients between the blanket structures and superconducting magnets. Temperatures change from $750^{\circ}-1250^{\circ} \mathrm{K}$ to $4^{\circ} \mathrm{K}$ over distances of the order of $1 \mathrm{~m}$. (The $\partial$-pinch does not use superconducting magnets and these large thermal gradients are not present.)

c) Liquid He in the cryogenic systems has a density of $125 \mathrm{~kg} / \mathrm{m}^{3}$. The vapor at the boiling point has a density of $16 \mathrm{~kg} / \mathrm{m}^{3}$ and at STP $0.17 \mathrm{~kg} / \mathrm{m}^{3}$. Helium can thus increase in volume a factor of 7.8 to vapor at the boiling point and a factor of 718 from 1 iquid to vapor at STP.

d) The plasma thermal energy is estimated at $500-4000 \times 10^{6}$ joules for a 5000 MWt plant. (3) The fusion of 1 mole of $T$ and 1 mole $D$ produces $1.7 \times 10^{12}$ joules of energy. Most of the plasmas contain $\sim 1 / 4$ mole $D$ and $T$. A complete reaction of the fuel is impossible. (3)

e) Tritium will probably be pressurized at some state during preparation for storage. Pressures are not specified.

f) The thermal energies in liquid metals were estimated using the temperature of the metal and a representative value for the heat capacity.

g) Blanket structures are estimated to contain about $1 \%$ or less of the reactor thermal output as decay heat (see discussion in text). (3)

h) The heat contents of steam and He coolants are based on mass flow rates and estimated thermal output through that system.

i) Steam cycle is not described. Information listed is for the Direct Conversion Cycle.

\section{REFERENCES}

1. B. F. Gore and E. S. Murphy, Current Fusion Power PIant Design Concepts, BNWL-2013, Battelle, Pacific Northwest Laboratories, Richland, WA, 1976.

2. J. O. Cowles and A. D. Pasternak, Lithium Properties Related to Use as a Nuclear Reactor Coolant, UCRL-50641, Lawrence Livermore Laboratory, Lawrence CA, 1969.

3. R. Bunde, et a1., Aspects of Energy Supoly by Fusion Reactors, BNWL-TR-140, translated from German by Battelle, Pacific Northwest Laboratories, Riciland, WA, Apri1 1975. 
APPENDIX C

SOME SAFETY IMPLICATIONS OF THE PRESENCE OF LIQUID ALKALI METALS IN FUSION REACTORS

Liquid $\mathrm{Li}$ is present in the UWMAK-I design at temperatures to $500^{\circ} \mathrm{C}$, the ORNL design at temperatures to $1050^{\circ} \mathrm{C}$, the theta pinch to $550^{\circ} \mathrm{C}$ and the mirror device at unspecified temperatures. Liquid $\mathrm{Na}$ is the secondary coolant in the UWMAK-I design $\left(500^{\circ} \mathrm{C}\right)$ and the theta pinch $\left(522^{\circ} \mathrm{C}\right)$. Liquid $\mathrm{K}$ at a temperature of $98^{\circ} \mathrm{C}$ is the secondary coolant in the ORNL tokamak. Liquid metals are highly reactive, therefore, their potential for initiating or propagating an accident is an important consideration in the design of fusion reactors.

Of greatest potential importance are the liquid metal reactions with air, water and concrete. Some information exists in the literature concerning these reactions, although the majority deals with Na as a result of its use in the LMFBR program. The chemical similarities of the alkali metals allow these results to be extrapolated in a qualitative sense to $L i$ and $K$, but some differences exist.

The most recent information available on liquid metal-concrete reactions is by Hilliard. (1) His results are for $\mathrm{Na}$ reactions but he indicates ${ }^{(2)}$ that $L i$ and $K$ reactions may not be significantly different. The major difference would probably be in the amount of energy released by the reaction. Sodium reacts directly with the solid constituents of concrete and also with the entrained water which is drawn out of the porous concrete. These reactions are presented as Equations 1 through 8 in Table $c-1$. (The hydrides formed by Equation 3 are probably unstable at the temperatures that could conceivably be encountered in accidents at fusion reactors.)

Hilliard reports that in reactions with $\mathrm{Na}, 75$ to 98 percent of the water will be drawn out of concrete to depths of one foot (the maximum thickness of concrete tested). At temperatures above the melting point of the hydroxide, ${ }^{(2)}$ the reaction given in Equation 2 will dominate the liquid metal reactions with water. In atmospheres less than 5 percent oxygen, the hydrogen gas produced will not be oxidized and has the potential to produce large pressures in closed areas. If the oxygen content is greater than 5 percent, the hydrogen may react explosively if ignited. (1) (The water vapor produced may also feed the fire from above.) Concrete-liquid metal reactions may be self-extinguishing by the accumulation of a surface layer of oxide and silicate, since the liquid metal must penetrate to the reaction site. Surface spalling through an unknown process may keep the reaction going to depths of 1 to 2 or more. If cracks develop in the concrete from thermal stresses, the reaction could proceed until one of the reactants has been consumed. Reactions with high density concrete proceed in a similar way. High density concrete contains about 85 percent iron oxides. The reactions of liquid alkali metals with iron produce about $50 \%$ less heat per mole of liquid metal reacted than the reactions with $\mathrm{SiO}_{2}$ in normal concrete. In present designs, steel liners over concrete surfaces which might be contacted by a spill of liquid metal would effectively prevent liquid metal-concrete reactions. 


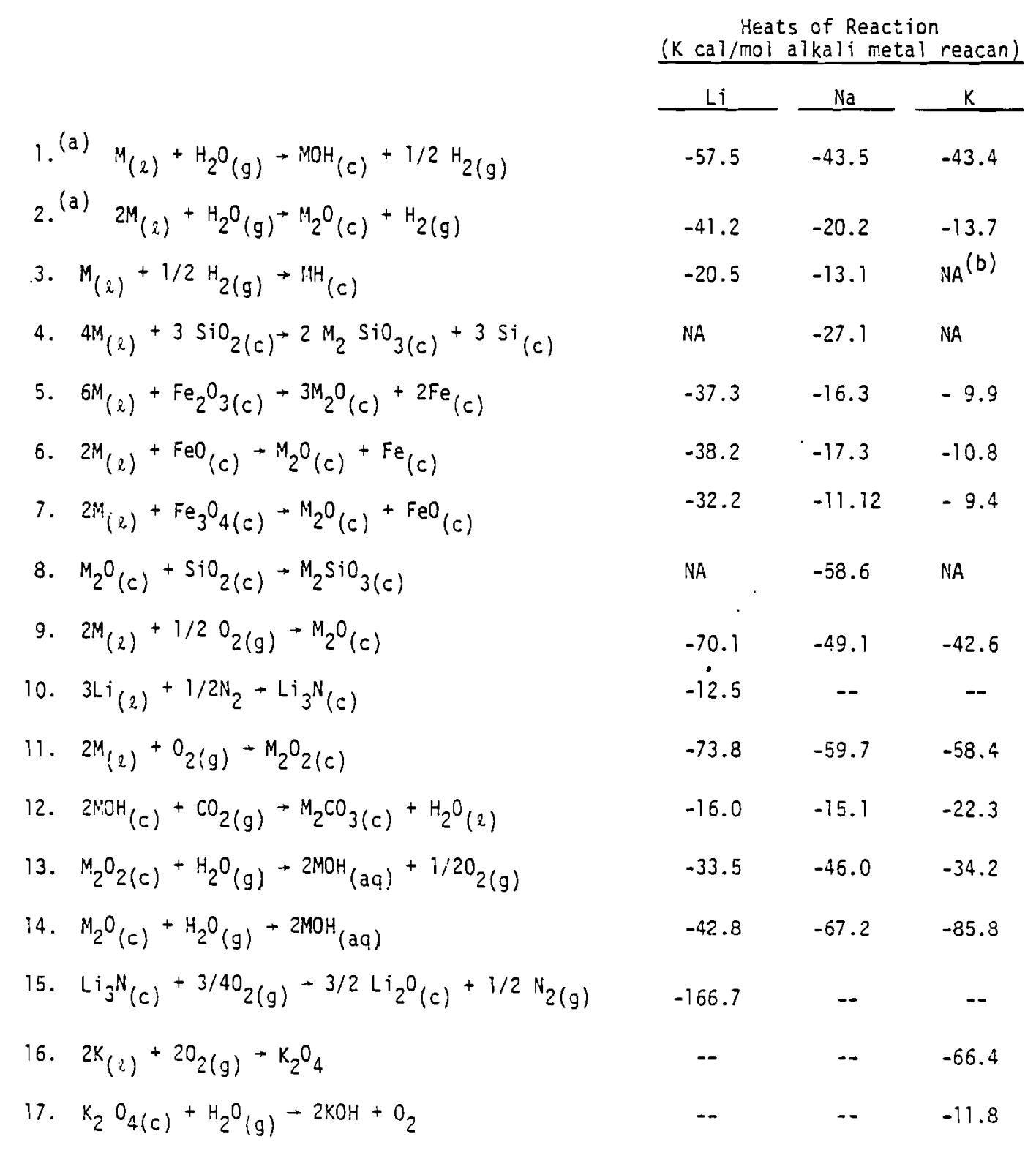

(a) Melting points: $\mathrm{NaOH} 318^{\circ} \mathrm{C}-\mathrm{K} \mathrm{OH} 360^{\circ} \mathrm{C}-\mathrm{LiOH} 450^{\circ} \mathrm{C}$

(b) Not Available

(c) Crystaline (Solid) 
Smoke generation from a concrete-liquid metal reaction is smal1. (2) With the exception of the evolved hydrogen gas, most of the reaction products are deposited in the surface layer mentioned above. Tables $\mathrm{C}-2$ and $\mathrm{C}-3$ (taken from Ref. 1) show the constituents and reaction products for Na reactions with normal and high density concrete. A differential thermal analysis by $\mathrm{Hi} 11$ iard for sodium indicates that reaction 4 in Table $\mathrm{C}-1$ begins at $538^{\circ} \mathrm{C}$, and reactions 5 and 6 at $464^{\circ} \mathrm{C}$. Liquid metal reactions with loose water or steam are identical to those discussed above for the water extracted from concrete. Generation of hydrogen gas during reactions is a primary safety consideration.

Fractional release values as high as one-third of the metal burned in an air fire being released as smoke (primarily in the form of metal oxides) have been reported. (2) There are indications, (3) that once released into the atmosphere, these oxides become hydroxides through reactions like 13, 14, and 17 shown in Table $\mathrm{C}-1$ and subsequently form carbonates by reactions 1 ike 12 . There is no information readily available on the toxicity of alkali metal carbonates, but they are relatively stable compounds and should be less harmful than the oxides or hydroxides. Hilliard notes $^{(1)}$ that liquid $\mathrm{Na}$ will burn in air at any temperature above the melting point. There is some evidence ${ }^{(4)}$ that Li will not ignite below $600^{\circ} \mathrm{C}$ unless some lithium nitride seed material is present to initiate the reaction.

Some differences in the liquid metal reactions with air are noted. Lithium will react with the nitrogen in air, and studies indicate ${ }^{(4)}$ that the Li-air reaction proceeds stepwise from reaction 10 to reaction 15 (Table $\mathrm{C}-1$ ). The density of liquid 1 ithium is small $\left(0.49 \mathrm{gm} / \mathrm{cm}^{3}\right.$ at $.400^{\circ} \mathrm{C}$ ). Combustion products may sink in the lithium, leaving a fresh layer of metal exposed to the air at all times. (This characteristic also makes extinguishment of a Li fire difficult.) Potassium has a tendency to form superoxides by reaction 16. Such superoxides are very reactive and would probably not be present in a release to the environment following a potassium fire.

Liquid 1 ithium will undergo some reactions that the other alkali metals will not. It reacts with all known molecular gases. At temperatures above $900^{\circ} \mathrm{C}$ it will react with carbon to form acetylides which readily hydrolize to form acetylene. (5) The presence of large amounts of graphite in some of the fusion reactor designs and explosive nature of acetylene may make this an important safety consideration.

Secondary effects of liquid metal fires and reactions with concrete may also be an important safety consideration. A large spill has the ability to consume significant amounts of concrete. Structural integrity of portions of the facility could be threatened. The energy releases involved in the reactions have the potential to damage or vaporize nearby materials. Radioactive material could be volatilized and potentially released to the environment in this manner.

From the information presented above, it can be seen that the consequences of a liquid metal spill may vary widely from plant to plant. Some of the reactions are temperature dependent. The spill environment will determine to a large extent which reactions actually do take place. The hydrogen and acetylene producing reactions are the primary safety concerns within the plant. 
TABLE C-2. Composition of Conventional Concrete (Type C1-P) and Reaction Products (Na-Concrete Reaction at $\left.\sim 1100^{\circ} \mathrm{F}\right)$

\begin{tabular}{lcc} 
& \multicolumn{2}{c}{ Weight, $\%$} \\
\cline { 2 - 3 } & $\begin{array}{c}\text { Fresh } \\
\text { Concrete }\end{array}$ & $\begin{array}{c}\text { Sodium } \\
\text { Reaction } \\
\text { Product }\end{array}$ \\
$\mathrm{SiO}_{2}$ & 54.5 & 3.2 \\
$\mathrm{CaO}$ & 15.2 & 4.2 \\
$\mathrm{AT}_{2} \mathrm{O}_{3}$ & 10.5 & \\
$\mathrm{FeO} \mathrm{Fe}_{2} \mathrm{O} 3$ & 6.6 & 9.7 \\
$\mathrm{MgO}$ & 3.2 & 0.8 \\
$\mathrm{Na}_{2} \mathrm{O}, \mathrm{K}_{2} \mathrm{O}$ & 3.4 & 60.0 \\
$\mathrm{SO}_{3}$ & 0.3 & 0 \\
$\mathrm{H}_{2} \mathrm{O}$ & 6.0 & 17.4 \\
$\mathrm{Ma}_{2} \mathrm{SiO}_{3}$ & 0 & 4.7 \\
Other & 0.3 & 100.0
\end{tabular}


TABLE C-3. Composition of Magnetite Concrete (Type M-225) and Reaction Products (Na-Concrete Reaction at $27100^{\circ} \mathrm{F}$ )

\begin{tabular}{lcc} 
& \multicolumn{2}{c}{ Neight, \% } \\
\cline { 2 - 3 } & $\begin{array}{c}\text { Fresh } \\
\text { Concrete }\end{array}$ & $\begin{array}{c}\text { Sodium } \\
\text { Reaction } \\
\text { Product }\end{array}$ \\
$\mathrm{SiO}_{2}$ & 2.4 & 1.7 \\
$\mathrm{FeO}, \mathrm{Fe}_{2} \mathrm{O}_{3}, \mathrm{Fe}_{3} \mathrm{O}_{4}$ & 85.6 & 41.7 \\
$\mathrm{CaO}$ & 6.6 & 2.8 \\
$\mathrm{Al}_{2} \mathrm{O}_{3}$ & 0.38 & 0.5 \\
$\mathrm{MgO}$ & 0.10 & 0.7 \\
$\mathrm{Na}_{2} \mathrm{O}$ & 0.04 & 48.1 \\
$\mathrm{SO}_{3}$ & 0.21 & 0 \\
$\mathrm{H}_{2} \mathrm{O}$ & 4.45 & 2.0 \\
$\mathrm{Na}_{2} \mathrm{SiO}_{3}$ & 0 & 2.5 \\
Other & 0.11 & 100.0
\end{tabular}


Combustion of liquid metals in air and the resultant release of oxides to the environment potentially represents the greatest environmental concern, although the consequences may be mitigated by the formation of carbonates as the smoke drifts away from the release point. Threshold limit values exist ${ }^{(6)}$ for only two of the possible reaction products discussed. These are $0.025 \mathrm{mg} / \mathrm{m}^{3}$ of air for $\mathrm{LiH}$ and $2 \mathrm{mg} / \mathrm{m}^{3}$ of air for $\mathrm{NaOH}$. These are industrial limits. Limits for public exposure have not been established. A great deal of the information necessary to assess the safety associated with the presence of liquid alkali metals in fusion reactors is not available. The reactions presented in Table $C-1$ have been confirmed for the most part only for sodium. The remainder have been inferred. Experimental investigations in support of fusion reactor design and safety analyses are needed in several areas including: 1) Li and $K$ fires and reactions with concrete, 2 ) the behavior of liquid alkali metal reaction products in the atmosphere, 3) the behavior of large scale $\mathrm{Na}$ fires and reactions with concrete, 4) toxicity of the liquid alkali metal reaction products to animals, plants and man, 5) design methods which could mitigate the effects of spills of liquid alkali metals.

\section{REFERENCES}

1. HEDL-SA-983, R. K. Hilliard, Sodium - Concrete Reactions, Liner Response, and Sodium Fire Extinguishment, Westinghouse Hanford Co., Richland, WA, July 1975.

2. Hilliard, R. K., Personal Comunication.

3. AERE-R6460, W. S. Clough and J. A. Garland, The Behavior in the Atmosphere of Aerosol from a Sodium Fire, United Kingdom Atomic Energy Authority Research Group, Harwe11, England, 1970 .

4. Development of Fire Extinguishing Agents for Li, Aurthur D. Little Inc., Dec. 1960.

5. UCRL-50647, J. 0. Cowles, A. D. Pasternak, Lithium Properties Related to Use as a Nuclear Reactor Coolant, Lawrence Radiation Laboratory, University of California, Livermore, CA, Apr. 1969.

6. Weast, R.C., ed., Handbook of Chemistry and Physics, CRC Press, Cleveland, OH, 1974. 


\section{R\&D NEEDS - SUPPLEMENTARY INFORMATION}

Analytical methods are required to predict the performance of fusion reactor designs relative to system safety. Additionally, experimental data are required to validate these predictions. This appendix provides the detailed information and the bases to support the areas of research and development needs for fusion reactors.

The logic developed and used to identify areas which require research and development is shown in Figure D.1. Within the framework of that figure, PNL staff, from a broad range of technical disciplines, identified potential safety concerns for magnetic confinement fusion reactors based on their experience in safety research. They used the following format.

- Safety Item (Event or phenomenon to be investigated.)

- Safety Concern (How the above item can impact on public or occupational safety?)

- Method of Analysis (Computer codes, plant simulation, what data-input, failure, etc., are needed?)

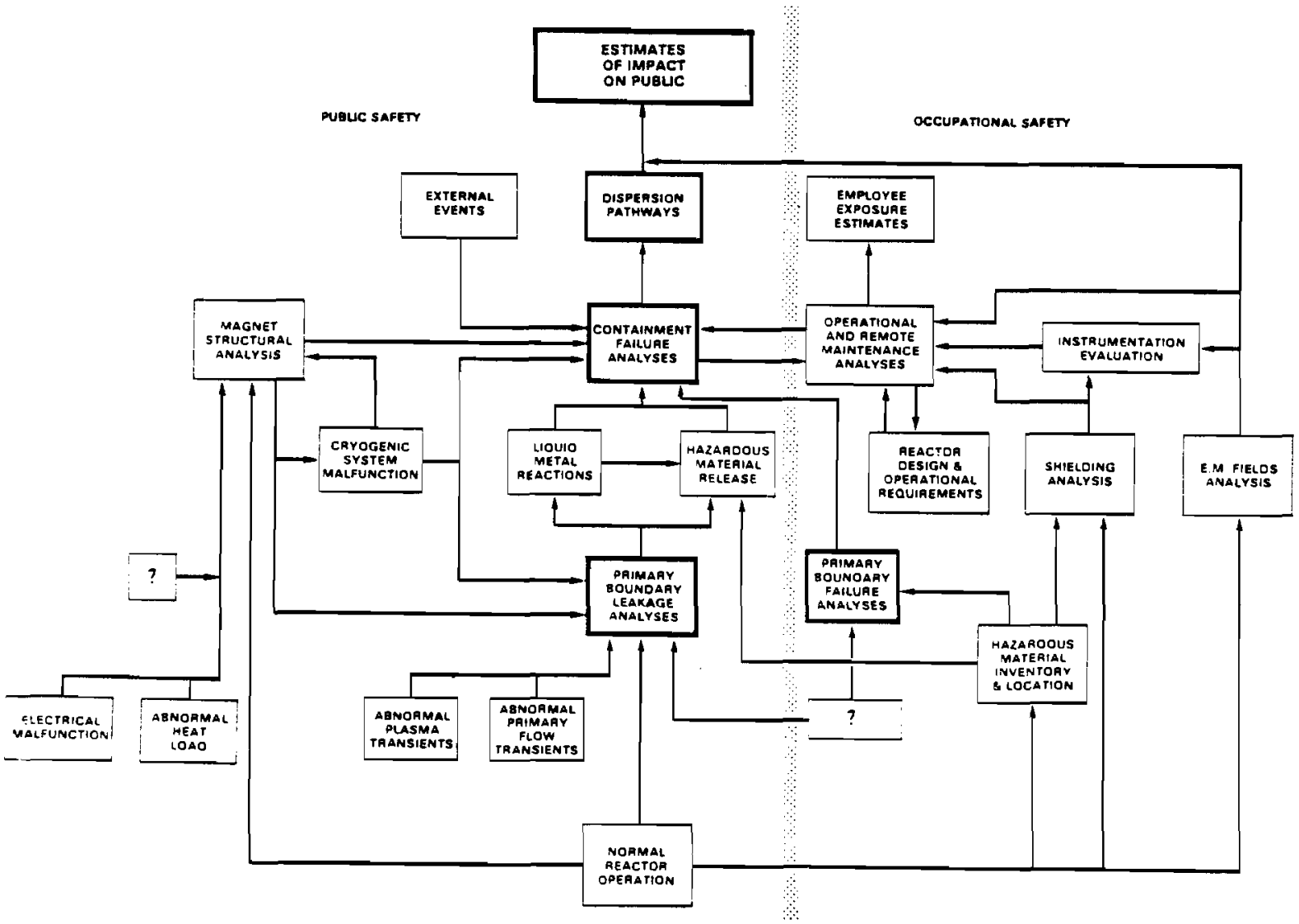

(The rectangles with question marks symbolize unidentified initiating events.)

FIGURE D-1. Safety Analysis Logic for Magnetic Confinement Fusion Reactors 
- Applicable Current Technology (What methods and data exist from fission reactor and/or aerospace technology appear to apply?)

- Needed for the Analys is

A. Design Information (What additional information is needed about fusion concepts before this analysis can be made?)

B. R\&D Requirements (What additional calculational tools, basic research and/or failure criteria must be developed before this item can be analyzed adequately?)

A compilation of their inputs follow, consistent with the logic in the blocks in Figure 0.1 . 


\subsection{PRIMARY BOUNDARY FAILURE}

Most major accident scenarios of fusion facilities will involve a failure of one of the primary boundaries and the subsequent release of potentially significant amounts of radioactive material. Therefore, it is important to identify potential failure modes in these boundaries. The data necessary to predict the limiting failure thresholds for the changes in corresponding parameters encountered during various accident scenarios must be developed. The primary boundary failure discussion thus identifies materials properties and component environmental parameters that must be determined to predict failure of a primary boundary.

The systems that will normally contain radioactive material are defined as:

- Primary Coolant Loop - including blanket components and exreactor components.

- Tritium Handling Systems - including extraction from primary coolant, storage and fueling.

- Vacuum System - including the first wall, divertors, beam ports and exreactor components.

The degree to which potential failure modes for components of these systems can be identified depends on how much design detail has been developed in conceptual studies performed to date. The following are preliminary analyses of the requirements for some components of these systems. They reflect not only accident conditions but also design, fabrication and operational requirements that impact on primary boundary failure and failure detection.

\subsection{Primary Coolant Loop}

\section{T.1 Coolant-Structural Material Interaction}

Safety Concern. (How above item impacts on public or operational safety). The interaction between coolant(s) and structural materials can be a prime degradation factor in the life of structural materials with obvious safety implications.

Method of Analysis. (Computer codes, plant simulation, data-input, failure, etc. needed?) Generally, theoretical analysis has been secondary to experimental revelation in the analysis of coolant-material interactions, primarily because of the number of factors involved and the complexity of the interaction between coolant and material. Loop studies utilizing the best possible simulation of operating conditions (temperature, impurity background, flow, stress background) will probably be the major recourse, apart from actual experience in this study. Lithium, in particular, presents many subtle problems of corrosion/mass transfer, in addition to the phenomenon of liquid metal embrittlement, all of which are sensitive to the impurity loading of the lithium.

Applicable Current Technology. (What methods and data exist from fission reactor and/or aerospace technology appear to apply?) Helium, water and lithium are being considered for various cooling functions in fusion concepts. The problems peculiar to water have been under analysis for many years in conjunction with fossil and fission powerplants. Helium (in particular helium impurities) alsc has considerable theoretical and experimental anaiytical precedence. Lithium presents probably the greatest anatytical challenge as well as the greatest threat to structural integrity. 
Needed for the Analysis

A. Design Information (What additional information needed about fusion concepts before this analysis can be made?)

1. Impurity loading of helium, water, lithium conceivable under operating conditions.

2. Identification of temperature-stress conjunctions where coolant-material interactions may be expected to be most severe.

3. Characterization of flow conditions that affect the mass transport of corrosion products and material-coolant interactions.

4. Fusion specific radiation field influence on coolant-material interaction.

8. R\&D Requirements (What additional calculational tools, basic research and/or failure criteria must be developed before this item can be analyzed adequately?)

LMFBR studies have shown that coolant exposure without some attempt at simulation of concurrent stress conditions can be virtually meaningless with respect to practical knowledge of material serviceability. Hence, test work should, where appropriate, attempt some synthesis of the full spectrum of actual working conditions, including loop and component tests.

\subsubsection{Primary and Secondary Piping System Integrity Surveillance}

Safety Concern. Rupture of primary piping system can result in loss of coolant flow to the blanket and release of lithium, tritium, and other activation products into the plasma region or containment system. Failure of secondary piping systems will result in contamination of secondary and heat exchanger systems.

Method of Analysis. The detection of cracking in piping systems is generally accomplished using ultrasonic and/or eddy current nondestructive testing techniques from either the ID or $O D$ of the tube or pipe.

Applicable Current Technology. Ultrasonic and eddy current techniques have or are in the process of being developed which would be suitable for inspection of water or helium piping. Piping for liquid metal coolants such as lithium can at present be inspected from only the 00 surface where accessible. Noncontacting ultrasonic devices (electromagnetic-acoustic transducers) are presently being developed and may prove feasible for ID inspection of Tithium piping systems.

Needed for the Analysis. An experimental program will be required to determine the effectiveness of nondestructive evaluation techniques in locating cracking or wall thinning in liquid metal piping systems before they result in a leak.

1.1.3 Remote Welding and Inspection of 316 Stainless Steel

Safety Concern. Weld joints are the primary closures for piping, nozzles and structural members. Experiences of LWR with 316 SS during construction and inservice nondestructive examinations and on FFTF with construction examinations have demonstrated difficulties with establishing and verifying the integrity of the weld. 
Since fusion must utilize remote techniques for repair, maintenance and modification of structural members, further development is needed before automatic remote welding and inspection technology can provide the weld integrity and safety required.

Method of Analysis. Present volumetric methods for establishing weld quality are based on radiographic (RT) and ultrasonic test (UT) methods. For LWR systems radiographic techniques are generally employed in compliance with Section XI. The Fast Flux Test Facility (FFTF) has utilized both methods during the construction phases, however, both have experienced limitations especially in the thicker sections. No volumetric techniques have been developed for inservice weld inspection on FFTF, however, detection systems are used to locate leaks. The limit of present nondestructive test methods lies in the grain size in the (HAZ) of the weld.

Applicable Current Technology. Weld inspection of 316 SS remains a difficult task. The acoustic emission (AE) test method is now being researched to improve the quality, analysis of welds. Preliminary research with stainless steel shows promise for detecting cracking caused by an improper weld procedure. A second area of research relates to improved welding procedures which provide a high quality weld which could accommodate RT, UT and AE to insure higher quality and safety of failure to critical structures.

Needed for the Analysis

A. Design Information

1. Identification of failure critical structures

2. Characterization of types of weids to be investigated

3. Nature of weld defects and their failure mechanism (fatigue, SCC, etc.)

4. Analysis of applicable nondestructive test techniques.

B. R\&D Requirements

1. An integrated remote welding and nondestructive test program and test facility is required. Improvements are needed to develop a weld in 316 SS which is more readily acceptable. Welds must be analyzed to determine the optimum remote inspection techniques.

2. Research is required to determine if acoustic emission technology can be developed to provide real time automatic feedback contro? to insure the welding process during the welding.

3. A test bed is needed to develop and evaluate both welding and inspection parameters.

\subsection{TRITIUM HANDLING SYSTEM}

See discussion of primary boundary leakage for some general comments on failure of components in tritium handling systems.

\subsection{VACIJUM SYSTEM}

\subsubsection{First Wall Failures}

Safety Concern. Plasma dump; load temperature excursion of vacuum first wa 11 and blanket structure; resulting in vacuum system overload or leakage of radioactive material to the containment bujlding. 
Method of Analysis. Potential design requirements as to normal and off-normal plasma and thermal loadings of first wall become "envelope" input to laboratory tests predictive performance code development relative to the combined effects of irradiation, themal and coolant environments on first wall degradation and failure.

Applicable Current Technology. A considerable body of knowledge from LMFBR studies can be applied; however, MFE conditions represent a substantial unknown regime; LMFBR (FFTF and CRBRP) licensing experience has shown that the development of relevant test data and predictive performance codes are required to satisfy public "health and safety" questions.

A. Design Information

1. Envelope of normal and off-normal events relative to first wall and their impact on first wall environmental loadings (flux, fluence, energy, temperatures, stresses, etc.).

2. Materials concepts (including coatings).

NOTE: It is anticipated that R\&D suggested below would feed back into improved design concepts.

B. R\&D Requirements

1. Development of "design-limit" (normal and off-normal) for first-wall materials (for example, maximum allowable strain per cycle and for total lifetime) based on the individual and synergistic effects of degradation processes (swelling, blistering, irradiation-creep, irradiation-embrittlement, thermal fatigue, high-strain rates, coolant environment, etc.).

2. Development of predictive performance codes to cover regimes inaccessible to laboratory studies, based on combining extensive laboratory studies for accessible regimes with theory.

3. Items 1 and 2 require R\&D that:

a. Investigates science of basic phenomena to deveiop theoretical understanding (should be covered by "non-safety R\&D).

b. Scopes the "accessible" performance limits of materials, base metal and weldments: covering the potentially limiting synergistic degradation effects (e.g., cyclic strain behavior and crack-growth rates of irradiation-embrittled and blistered material in flowing lithium).

c. Scopes the "accessible" performance 1 imits of potential structural configurations, through relevant $R \& D$ (from $a$ and $b$ ) and small-scale, simulated component test.

d. Develops predictive models of normal and off-normal component performance.

e. Develops a material surveillance program for early fusion reactors to obtain in-situ data. 


\subsubsection{First Wall Integrity Surveillance}

Safety Concern. Material property degradation in the first surface of the blanket resulting from neutron bombardment, thermal and pressure cycling may result in premature failure of the primary coolant system. A leak in the primary coolant system will release lithium, tritium and other activation products into the plasma region or containment system.

Method of Analysis. Nondestructive evaluation techniques are available for the determination of material property degradation.

Ultrasonic and electromagnetic (eddy current) techniques can be applied to determine the extent of material degradation resulting from neutron bombardment, thermal and pressure cycling. These measurements could be accomplished before operation and during periodic maintenance. The ability to perform these measurements would provide significant information for determining the suitability of the blanket for continued operation and its projected useful service lifetime.

Applicable Current Technology. Limited experiments have been performed on 316 stainless steel to determine the influence of neutron induced swelling and damage on the propagation of ultrasonic and electromagnetic waves. Results of measurements to date on a limited number of samples inidcate that a qualitative assessment of damage can be made. Controlled experiments will be required to determine the effectiveness of the techniques for obtaining quantitative information under the neutron influence and temperature ranges expected in operation.

Needed for the Analysis. An experimental program is required to determine the most suitable measurement technique for the determination of material property changes. Suitable samples containing various degrees of damage and the expected range of material and process variables would be required. From this measurement program the most suitable measurement technique would be selected and the configuration of testing systems for preservice and inservice inspection defined. 


\subsection{ABNORMAL PLASMA TRANSIENTS}

Abnormal plasma transients could in some way cause overheating of the first wall. Direct impingement of hot particles or excessive neutron loading and/or gamma radiation are potential sources of concern. A11 major plasma devices operated to date have experienced first wall damage. Programs must be started to characterize the basic phenomenon so it and the consequences are predictable.

The following writeups in this section are examples of programs that must be developed to assess the safety aspects of potential plasma transients. The plasma overpower transient is discussed from two points of view. In one the transient is assumed to occur on a time scale that allows cooling of the first wall. The other assumes a time scale that, in effect, adiabatically heats the first wall. This points out the importance of characterizing the possible modes of plasma overpower.

\subsection{PLASMA OVERPOWER}

Safety Concern

By an accidental increase of the magnetic field, which would compress a fusion plasma, the temperature of the plasma would be raised thereby increasing the specific reaction rate and the total power. This could then result in an increase in radiation and particle bombardment on the first wall and an overall increase in blanket temperature.

\section{Method of Analysis}

Erosion and damage to the first wall are caused by radiation and particle bombardment. Therefore, such plasma-wall interactions need to be addressed from a fundamental as well as engineering point of view. In order to understand plasma behavior and wall interaction, a knowledge of relevant atomic and molecular processes is necessary. As far as engineering is concerned, the rate of wall damage and deterioration by various processes has to be measured to determine the life limiting parameters of the first wall and other structural components, particularly under accident conditions.

\section{Appl icable Current Technology}

Since so far the break-even point for thermonuclear reactions has never been reached, no fusion plasma technology exists at present to generate experimental erosion data for the temperature range beyond the ignition point. However, the thermal power density at the first wall could be simulated by other (nonnuclear) devices. ( 1,2$)$ Effects of purely nuclear radiation could be investigated in an irradiation testing facility such as proposed by HEDL. A combination of both thermal and nuclear radiation effects in one facility would be desirable, however.

Needed for the Analysis

D-T reaction rate as a function of temperature has been estimated by a rather crude theory. According to this theory the reaction rate will decrease with temperature after passing through a maximum, thus making the fusion reaction inherently safe from a runaway power accident. 
However, refinement of this theory and its experimental verification on a quantitative basis is required before a fusion reactor could be licensed.

\section{Needed for the Analysis}

In particular, the following items require further analysis and R\&D:

A. Design Information

1. Detailed layout of first wall and blanket including design details of heat removal system.

B. R\&D Requirements

1. Influence of magnetic fields (steady and nonsteady) on plasma-wall interactions.

2. Thermal and particulate radiation as a function of plasma temperature and impurities.

3. Effect of such radiation on materials (up to and beyond the structural integrity limits).

4. Materials properties and inelastic behavior at high temperatures and under high stresses.

\subsection{PLASMA OVERPOWER}

\section{Safety Concern}

Overpower in the plasma would cause excessive heating of the first wall which may affect its integrity. The first wall failure may release coolant to the plasma region, including lithium/ tritium/activation products.

Plasma accidents are in view. Space-time rate of first wall energy deposition must be specified and coupled to coolant system simulation. First wall must be modeled in detail so that temperatures and stresses may be calculated accurately.

Need failure criteria for first wall and coolant system.

Applicable Current Technology

Available mechanics codes and heat conduction codes are applicable.

Needed for the Analysis

A. Design Information

1. Detailed blanket cooling systern design.

2. Accurate specification of energy deposition on first wall as a function of time and space.

3. Thermal and mechanical properties of first wall and bianket materials in a fusion reactor environment.

B. R\&D Requirements

1. Thermal and mechanical data for first wall materials.

2. Determine energy spectra to first wall from above conditions.

3. Determine conditions for overpower, instability, other plasmas abnomalities.

4. Failure criteria for first wall, coolant system and blanket structure. 


\subsection{PLASMA DUMP}

\section{Accident Safety Concern}

Experience with existing plasma devices has shown that the plasma is not always remaining in its intended configuration (such as symmetrical circular loop in a tokamak device), but oscasionally breaks out and comes into contact locally with the physical limits or wall where it will "dump" its energy. This will cause a local hot spot in the first wall of a fusion reactor which potentialiy could be so severe that it would result in permanent damage and eventual perforation of the wall. This, in turn, could then trigger additional accidents, e.g., coolant leakage into the torus.

\section{Method of Analysis}

The maximum amount of energy dumped into the wall depends on the total amount of fuel (e.g., $v 2$ grams in the reference reactor design) and its temperature. By analysis it has to be determined how fast and how much of this energy can be dumped into a limited area of the first wall without causing serious damage. Existing theory in this field is scant. Experience with actual cases of plasma dumps should be gathered and evaluated to substantiate and improve on the theory. New experiments guided by the preliminary theory should be undertaken.

\section{Applicable Current Technology}

Since plasmas at full temperatures have not yet been generated, only simulation experiments can be applied to the problem. Because plasma densities for fusion reactors are quite low, the same heat flux q could be obtained in a test with a plasma of lower temperature but higher density, since $q \sim T \sqrt{\rho}$. Test devices of such kind are available in the form of plasma jets, which have been used extensively in test facilities for the space program (e.g., for re-entry simulation). Needed for the Analysis

Theoretical treatment of the severe thermal transient stresses, ablation and possibly perforation of the first wall, which might occur as a result of a hot plasma dump, is not sufficiently advanced for a reliable analytical design evaluation required for licensing purposes. Therefore, plasma instabilities and resulting wall impact phenomena must be studied further, both theoretically and experimentally.

8. R\&D Requireinents

1. Cause and behavior of plasma macroinstabilities resulting in a wall contact.

2. Effects of a plasma dump on various wall materials.

3. Investigation of ways and means to prevent a plasma dump.

4. Influence of magnetic fields on a plasma dump phenomenon.

5. Effects of first wall design details on severity of potential accidents following a plasma dump.

6. Material properties and inelastic benavior under hot plasma impact. 


\subsection{CRYOGENIC SYSTEM MALFUNCTIONS}

Performance of the cooling system for the superconducting magnets will be of concern when assessing the safety of fusion facilities. There are three general areas in which the cryogenic system can impact on plant safety. The obvious problem of maintaining adequately low temperatures in superconducting materials is of prime concern. However, substantial leakage from the cooling system could cause failure of the primary boundary due to themal shock and overpressurization of the containment if sufficient energy is transferred to the liquid helium.

The following are specific examples of development programs that will be necessary to estimate the impact of the cryogenic cooling system on plant safety.

\subsection{AIR LEAKAGE INTO THE LIQUID HELIUM DEWAR OR INSULATING SPACE}

\section{Safety Concern}

Air solidifies when it comes into contact with liquid or low temperature gaseous helium. The solid air could plug coolant lines or passages. Upon heating the system for maintenance or other purpose, an explosive mixture of liquid hydrogen and solid oxygen could be formed. Very little energy would be required to set this mixture off. Later, as the liquid nitrogen-oxygen mixture is vaporized, liquid oxygen becomes concentrated in the liquid phase. This concentration of oxygen can cause the burning of compounds which would not otherwise ignite.

The leakage of nitrogen or air into the insulating space creates two hazards. Both could result in an explosion due to the vaporization of a liquid in a confined space. One hazard is the creation of a heat leak and the subsequent rapid heating of liquid helium. The other hazard is the entrapment of solid or liquid air in the insulating space and subsequent ballooning or expansion of the insulating space when it is heated to room temperature.

\section{Method of Analysis}

The effect of flow blockages on temperature and pressure responses of the magnets can be determined from the use of modified varsions of existing thermal-hydraulic codes.

Thermal code could be used to determine the magnitude of potential heat leak due to the leakage of air into an insulating space.

The potential for air leakage into the liquid helium would need to be modeled as would the expansion of the gas and the resuitant pressure buildup.

\section{Applicable Current Technology}

The basic flow modeling method currently used to analyze fission reactor loss of flow accidents are also applicable for this case. Existing thermal codes can be used to determine the magnitude of heat leaks. Stress code could be used to determine potential structural damage. 
Needed for the Analysis

A. Design Information

1. Detailed layout of the coolant system for the superconducting magnet system.

2. Detailed layout of the coolant circuits in the superconducting magnet including flow paths and headering, etc.

3. Detailed layout of the construction of the thermal insulation and dewar supports.

4. Mechanical and thermal properties of materials.

B. R\&D Requirements

1. Determination of insulation techniques

2. Develop a simulation of air leakage into helium and insulation spaces.

3. Determination of insulation degradation due to vapor pressure increase.

\subsection{LOSS OF MAGNET COOLING FLOW}

\section{Safety Concern}

Potential explosive rupture of the magnet dewar may cause damage to the magnet, the reactor blanket and shield, surrounding structure, and the containment building. The rupture would release large quantities of noncondensible inert liquid and vapor into the containment vessel. The released vapor would rapidly decrease the concentration of oxygen to dangerous levels in the containment building. The containment would have to withstand increased pressures resulting from release vapors.

\section{Applicable Current Technology}

A method for determining the thermal hydraulic response of various flow configurations has been developed. The resulting codes have been used to analyze the failure potential of power plant concepts. These codes could be modified to determine the temperature and pressure response of the cryogenic coolant used in superconducting magnet concepts. The response of the magnets to various accident scenarios could be determined.

\section{Needed for the Analysis}

A. Design Information

1. Detailed layout of the coolant system for the superconducting magnets including elevations of components, heat exchanger, pipe sizes, and refrigeration method.

2. Detail layout of coolant circuit in the superconducting magnets including flow paths, headering, etc.

3. Magnet design detail including material, transition temperature, superconducting and normal resistances, and operating current and voltage. 

4. Flow and power control system
5. Pressure relief system
6. Thermal insulation design

B. R\&D Requirements

1. Determine thermal and mechanical properties of the structural materials associated with the magnet, thermal insulation and containment.

2. Determination of the thermo-physical properties of helium over the temperature and pressure regions of interest.

\subsection{LOSS OF CRYOGENIC COOLANT}

\section{Safety Concern}

Displacement of air and oxygen causing a safety hazard for personnel. Large volumes of air can be rapidly displaced by vapor resulting from the spilling of small quantities of cryogenic liquids. The cryogenic liquid vaporize quickly as they come in contact with the warm environment. Also, direct contact with the fluid could cause frostbite.

A possible failure of structural members resulting from their being cooled by the escaping cryogenic coolant. The failure may occur due to intense themal stressing or a change in material properties. (Some structural materials become brittle at low temperatures.)

Method of Analysis

The escape, vaporization, and flow path of leaking cryogen would be difficult to model. One of the primary concerns would be to safeguard the lives of the operators. This could be done by the proper design and location of detection units to warn the operator of low oxygen concentrations.

The effect of cooling of the structural members could be modeled by the use of existing thermal stress codes.

Applicable Current Technology

Methods used to detect and prevent leaks in commercial liquification plants can be applied. Available thermal-stress codes can be used in the analysis of potential structural problems. Needed for the Analysis

A. Design Information

1. Detailed layout of the magnet and containment vessels and their relationship to other components.

2. Size and location of containment ventilation systems

3. Detailed layout of cryogenic coolant circuit. 
4. Location of all personnel access areas with escape routes.

5. Identification of structural materials.

B. R\&D Requirements

1. May require the strength characteristics of some structural materials (materia) that may be used but property data are not available at low temperatures). 


\subsection{CONTAINMENT ANALYSES}

The performance of the containment building and cleanup systems will be very important to both the public and operational safety of fusion facilities. Three general types of analyses must be made. First, knowing the radioactive material released due to leakage or failure of a primary barrier, it is necessary to perform dispersion calculations to predict the concentrations of radioactive material throughout the plant. These calculations include plate out of condensable materials and removal of tritium and activation product by the gaseous and liquid waste processing systems.

The concentrations then become source terms for shielding calculations that predict the ionizing radiation throughout the critical access areas of the plant. The shielding calculations are also performed for both accident situations and normal operation.

A structural analysis must also be made to estimate the leakage of radioactive material from the containment building. This would include determining the building response to natural disasters as well as external and internal missile penetration. The estimated leakage plus the discharge from the effluent control system yields the total amount of radioactive material dispersed to the public during normal operation or as the result of an accident.

The following discussions indicate some specific example of R\&D required to accomplish the containment analyses.

\subsection{CONTAIMMENT STRUCTURAL ANALYSIS}

\section{Safety Concern}

Containment systems of a fusion reactor differs from a fission reactor in that in a fusion system the containment structure is subjected to cyclic neutronic fluences, cyclic magnetic loads and partial vacuums that may have to tolerate overpressures. The end safety concern is the breach of the containment system and the ensuing release of an unacceptable amount of tritium. Method of Analysis

Determine what the effect is of cyclic neutron flux combined with magnetic induced stress upon a reinforced concrete containment system. Fully define the magnetic field inside the containment system and determine the effect of a magnetic ( $316 \mathrm{~L}$ stainless can under certain cyclic stress conditions, become martensitic and hence magnetic) body becoming free inside the containment volume.

Applicable Current Technology

Large structural computer codes and computer codes dealing with tornado and missile impact and experience gained during experiments dealing with containment systems in fissile reactor systems are available and can be used. 
Needed for the Analysis

A. Design Information

1. Material behavior of concrete under cyclic stress and neutron flux conditions.

2. Fairly well defined concepts of containment systems and internal magnetic fields.

B. R\&D Requirements

1. Material property tests of composite, stressed elements of containment system.

2. Development of computer codes to correlate with experimental data and allow prediction of long-range behavior of different designs of containment systems.

\subsection{SOLID MATRIX RADIOACTIVE WASTE RECYCLING}

\section{Safety Concern}

With the economic and resource conservation pressures that are bound to be put upon operators of such facilities because of the nature and quantities of the materials involved, a detailed analysis of recycling potential should be made. These recycling scenarios potentially have a large impact on personnel and public radiation exposure.

Method of Analysis

With the experience which has been gained in the advanced Waste Management Study on postulating feasible scenarios, analyzing radiological and economic impacts and identifying needed technology development, it seems reasonable to utilize similar methods of analysis in this case.

\section{Applicable Available Technology}

In the initial phases, this study is envisioned to be strictly a data gathering, paper study, with experimental research following as needs are identified. Therefore, initially the advanced Waste Management Project concept would be utilized. In addition a great volume of work on nonradioactive materials recycling is presently being done and will undoubtedly be applicable. 


\subsection{ABNORMAL PRIMARY FLOW TRANSIENTS}

There are several potential accident scenarios that are initiated by abnomal primary coolant flow or temperature transients. The transients discussed here are limited to the primary system, however, there are also perturbations to the intermediate coolant and power conversion systems that will have to be examined to demonstrate safe plant operation has been achieved by the designers. This latter category of transients will closely follow those currently analyzed to demonstrate the adequacy of relief and safety valves and overall plant control systems.

\subsection{LOSS OF BLANKET PRIMARY COOLANT FLOW}

Safety Concern

Loss of blanket coolant flow to one or more blanket segments may cause failure of the primary coolant system thus releasing lithium, tritium and other activation products into the plasma region or containment system.

\section{Method of Analysis}

Methods for determining plant response to loss of flow accidents have been developed for most power plant concepts. In general a simulation of the heat removal system is required. Critical temperatures and/or pressures are identified and models to predict them are included in the simulation. In general a fairly detailed description of the heat removal system is required to develop the simulation input. Applicable plant control models are included.

Potential failure criteria must be known to evaiuate the plant response once it is known.

Applicable Current Technology

The basic modeling methods currently used to anaiyze fission reactor loss of flow accidents are also applicable to blankets. Strength and ductility data for irradiated stainless steels are available for use for concepts employing them as structural material.

\section{ileeded for the Analysis}

A. Design Information

1. Detailed layout of heat removal system including elevation of components, heat exchanger sizes, loop interconnections, pipe sizes.

2. Detailed layout of coolant circuit in the blanket including flow paths, headering, etc.

3. Primary loop pump characteristics

4. Flow-power control system

5. After heat due to activation products

6 . Total neutron fluence $>1 \mathrm{MeV}$ on critical structural components 
7. Magnetic field lines as a function of time during the operating cycle from all significant sources.

8. R\&D Requirements

1. Liquid metal flow in changing magnetic fields to inciude in momentum balance for hydrodynamics model.

2. Irradiated stainless steel strain-rate failure criteria.

3. Develop simulation of blanket heat removal systems including all important phenomenon.

\subsection{LOSS OF EXTERNAL THERMAL BALLAST OR COOLAINT FLOW CONTROL}

\section{Safety Concern}

Resulting cyclic temperature excursion may fail piping, blanket structure, heat exchanger. Possible release of primary coolant.

Method of Analysis

Entire cooling system would be modeled in detail including blanket, external primary and secondary systems, themal ballast and control system. System response to several power cycles would be determined without thermal ballast. Critical temps and pressures would be identified and failure criteria applied.

\section{Applicable Current Technology}

Many loop codes available. Probably require some modification.

Needed for the Analysis

A. Design Infomation

1. Detailed coolant system design

2. Accurate specification of power cycle characteristics for the coolant system

3. Thermal and mechanical properties of blanket and structural materials.

B. R\&D Requirements

1. Simulation of blanket coolant systems

2. Determine thermal ballast requirements for pulsed systems

3. Envelopment of failure criteria for blanket components. 


\subsection{MAGNET STRUCTURAL ANALYSIS}

Maintaining the structural integrity of the magnets will be of prime concern in assessing

safety of fusion systems. Large amounts of energy wil1 be stored in the super conducting magnets. This fact along with the close proximity of the magnets to the primary and secondary containment boundaries, could lead to a total breach of containment if failure modes resulting in high velocity missiles can be proposed. Understanding the magnet structural response to off design conditions such as coolant system malfunctions, abnormal heat loads and electrical malfunctions will be necessary.

The following concerns point out some areas that are important to determining the parameters necessary to put magnet response into the context of public and operational safety.

\section{1 MAGNET STRUCTURAL FAILURE}

\section{Safety Concern}

Failure of magnet support structure can be construed as the allowance of sufficient distortion of the magnet itself to cause blockage of coolant flow paths leading to possible rapid quench and rapid release of energy. This latter action could in turn cause very rapid pressure build up within magnet cryostat resulting in explosive disassembly with damage done to the rest of the fusion system through missile action. Safety concern revolves around immediate effect-missile impact on personnel and secondary effect-release of tritium.

\section{Method of Analysis}

Gross failure of magnet support system sannot occur except by imposition of some accident scenario such as airplane crash, etc. . . . Normal design procedures will take care of prevention of nonaccident gross failure. What is needed here is the detailed analysis of the magnet system performing as a heterogeneous structure acted upon by the magnetic and pressure generated loads. These loads must be taken as cyclic and any tendency for the magnet internal structure geometry to change as a function of time must be fully determined to evaluate the change in the cooling channel characteristics.

\section{Applicable Current Technology}

Large, digital computer structural codes presently exist which can be applied to this problem. However, much material data is needed to define the performance of materials such as Niobium-tin, Niobium titanium, epoxy, copper, and stainless steels under a liquid helium environment and subject to cyclic, long time neutronic fluences. Much development needs to be done to reduce the very long effort and machine (computer) running times needed to carry out deformation studies once the material propertios are known.

Needed for the Anaiysis

A. Design Information

1. Material properties of magnet components under liquid helium temperatures and cyclic, iong time neutronic fiuences. 
2. Fairly detailed magnet designs, including support structures.

B. R\&D Requirements

1. Material property tests

2. Development of better software to simplify analyses of high heterogeneous structures.

\subsection{BEHAVIOR OF ORGANIC INSULATORS}

\section{Safety Concerns}

The safety concerns are primarily secondary effects. A thorough analysis should be made to determine the consequence of an instantaneous, perhaps explosive, failure of all organic insulators in the superconducting magnets during warming of the system.

Method of Analysis

Experimental data are required to determine the behavior of candidate organic insulators irradiated at cryogenic temperatures. If energy storage in the polymers is confirmed, modeling of the reactor system will be required to determine the consequences of instantaneous release of the stored energy on the system.

\section{Applicable Current Technology}

Little is known at present about radiolysis of organic polymers at cryogenic temperatures. The high energy radiation produces ions, free radicals, and other energetic species at all temperatures. At normal temperatures these species are short lived and react with other parts of the molecule or with other molecules causing cross-linking, polymerization, or degradation. However, at liquid helium temperatures, diffusion rates and reaction probabilities are so low that energy may be stored in the system in the form of trapped radicals or ions. Upon heating this excess energy would be available for release which may occur explosively when permitted to warm to the point where reaction can occur. Experiments need to be initiated to evaluate the potential for energy storage in polymers under fusion reactor conditions and the potential for release and the kinetic parameters determined for a variety of candidate polymeric insulators.

\section{Needed for the Ana lysis}

A. Design Information

1. Design of the superconducting magnets, particularly the insulators will be required.

2. The decision to proceed with organic insulators should be made soon so that the safety analysis can proceed.

B. R\&D Requirements

1. Radiation of polymers at cryogenic temperatures is required. This work can best be accomplished in an electromagnetic radiation flux. Neutron irradiation is not required at this time. Cryostats, however, would be required in conjunction with irradiation facilities to study the polymers. Currently irradiating in accelerators appears to be the most feasible approach. 


\subsection{ABNORMAL MAGNET HEAT LOAD}

There are potential accident scenarios which begin with abnormal heat loads to the magnet cooling system. These vary in severity from increased neutron and gamma loading due to abnormal plasma conditions to the effect of voided blanket and shield cooling passages. The analyses would utilize the design shielding methods with different source terms and/or blanket and shield compositions. The coolant system response would be determined by methods discussed in the Cryogenic System Malfunction section. It may be necessary, however, to more exhaustively identify more potential sources of thermal over load such as primary ccolant leakage, etc. This will be more practical when more design detail is availabie. 


\subsection{MAGNET ELECTRICAL MALFUNCTIONS}

Toroidal geometries result in somewhat symmetrical loading of magnet structures. There are, however, potential electrical malfunctions which could change this load pattern. The structural response of the magnets to other than normal magnetic forces will be important to assessing fusion power plant safety. Specifically what these malfunctions might be depends on the methods of startup and control that evolve in the designs. Therefore, it will be necessary for the designer to evaluate potential problems, solutions and R\&D requirements as part of the magnet design program. The safety program will have to verify that the design and accident analysis methods used are adequate to ensure plant safety. 


\subsection{NORMAL REACTOR OPERATION}

The design analysis of a fusion reactor during normal operation will quantify all of the parameters which characterize the fusion environment for all plant components and operating personnel. In a safety assessment of fusion facilities, it will be necessary to document the data base for all calculational methods used. This will be necessary to verify that the primary boundaries, magnet structure and cooling systems and the plant control systems have been adequately designed to prevent accidental release of significant amounts of radioactive material during normal operation. It will also be necessary to verify that the working environment for operating personnel has been adequately characterized. It will be the burden of the safety program to be sure the design bases are adequately documented. The bases themselves, however, will be developed as part of the Plasma Engineering, Magnet Design and Blanket Engineering programs. No attempt is made in this report to specify specifically all the design bases that will have to be documented. Suffice it to say that all calculational methods that impact on magnet, primary boundary or secondary containment integrity will have to be verified. The same is true of a 11 methods used to predict the EMI fields and toxic material exposure of plant personnel and the public. 


\subsection{PRIMARY BOUNDARY LEAKAGE}

The purpose of these analyses will be to combine the Teakage of the primary boundary from all sources identified by component designers. The results are the radioactive materials source term for the containment analyses. In addition, the leakage of liquid metals will be important to assessing operational safety.

Program requirements for estimating tritium leakage are identified in the discussions of tritium handing and storage in the Radioactive Materials Inventory and Location Section. The following discussion is indicative of some other concerns that will develop as CTR operation is defined.

\subsection{BLANKET LEAK DETECTION}

\section{Safety Concern}

Leaks in blanket are both an operational and a potential safety concern especially in liquid lithium coolant designs.

\section{Method of Analysis}

Helium leak detection techniques are commonly used to detect and locate minute leaks. The technology may be used to evaluate the blanket plasma surface during preservice examination and with special manipulating devices has some potential for inservice testing. Accuracy of pinpointing leaks behind the front wall of plumbing embedded in the blanket structure appears extremely difficult. In addition to being slow, the present approach can only locate leaks within a few centimeters (back wall). Helium detection processors are not applicable for continuous monitoring. A rapid accurate means is needed.

\section{Applicable Current Technology}

Coolant tracer tagging is one of the proposed means for speeding the detection process and is one step to developing an improved system. Acoustic emission technology, applied to leak detection, has potential for preservice and inservice inspection and with emphasis on sensor development has the potential for continuous monitoring. Computer triangulation acoustic emission techniques can pinpoint leaks accurately and quickly.

Needed for the Analysis

A. Design Information

1. Definition of size of leaks which are considered critical.

2. Description of the size of leak that must be detected.

3. Identification of locations where leaks are considered most serious (priority listing)

4. Analysis of the acoustic spectrum produced by liquid lithium leaks.

B. R\&D Requirements

1. Development of high temperature sensors for detecting acoustic emission for acoustic signature) 
2. Analysis of computer technology required to adapt known technology to the complex structure.

3. Further refinement of computer analyzer and software systems to improve resolution of sys tem.

4. Demonstration model of computer analyzer and acoustic emission to evaluate applicability of system for preservice, inservice and continuous monitoring for leaks. 


\subsection{SHIELDING ANALYSES}

The shielding analyses will define the ionizing radiation throughout the plant due to both high energy neutrons which leak through the primary shielding and the presence of radioactive material. Radioactive material includes both that which is circulating throughout the primary containment systems and that which has leaked into the secondary containment.

The results are important for both determining employee exposure during plant operation and for determining instrumentation requirements and adequacy. 


\subsection{ELECTROMAGNETIC (EM) FIELDS ANALYSIS}

It will be necessary to characterize the em fields during normal operation both within the plant and surrounding the plant. The first case is important to determining the affects on employees and plant instrumentation and control. The external fields are important to assessing public safety and environmental impact of fusion facilities.

It is anticipated the methods to perform these analyses will be developed as part of the Magnet Design Program. The safety program will have to make the necessary adaptations and document the adequacy of the methods. 


\subsection{INSTRUMENTATION EVALUATION}

There are two aspects of instrumentation which are important to assessing fusion reactor safety. First is the development of instrumentation that will characterize the electromagnetic interference (emi) fields in the plant. Secondly, what is the impact of the emi fields present in a fusion power plant on the performance and life of the diagnostic and control instrumentation proposed for ensuring operation of the plant.

The following are specific examples of tasks that will have to be carried out either as part of the safety program or a control and instrumentation program.

\subsection{FIRE DETECTION AND PROTECTION}

\section{Sa fety Concern}

Past experience with large accelerators has shown that detection of fires by conventional means in the presence of high electric, magnetic and radiation fields poses problems which must be considered in the design and operation of a fusion power plant. Malfunctions related to both false alarms and failure to respond have been encountered.

\section{Apol icable Current Technology}

Experience with some aspects of this safety item is being accumulated in the larger accelerator installations. Liquid metal coolant systems are being investigated as part of the breeder reactor safety program.

Needed for the Analysis

An in depth review of available information on fire detection and protection in the presence of electric, magnetic and radiation fields is needed. Subsequent to the availability of this information, an extension of the basic concepts should be made to the anticipated intensities of electromagnetic fields to be encountered in a full scale fusion power plant and this should be coupled with the potential of liquid metal fires within such a facility.

Unavailability of fundamental information in the literature may require the conduct of basic research in this area.

\subsection{AIR SAMPLING}

\section{Safety Concern}

Wigration of radioactive airborne particulates under the influence of intense electric or magnetic fields could seriously affect commoniy practiced precepts of particulate air sampling where particulate behavior can be predicted on gravitational and ventilation bases.

Method of Analysis

A simulation of anticipated electric and magnetic field intensities and spatial distributions would be necessary in the investigation of impacts of possible interference phenomena on particulate air sampling. 
Appl icable Current Technology

Simulation of electromagnetic fields, production of airborne particulate matter and sampling technologies necessary for this study are all in place for immediate use.

Needed for Analysis

Potential particulate air sampling interference from electromagnetic fields will necessitate a thorough analysis of the spatial distributions of the fields expected throughout the facility.

\subsection{HIGH ENERGY NEUTRON DOSIMETRY (14 MeV)}

Safety Concern

Personnel protection (occupational safety)

Method of Analysis

It is quite possible that the neutron energy spectrum of fusion reactors can be simulated using conventional accelerators. These devices should be adequate to establish methods and devices for dose equivalent measurements.

Applicable Current Technology

Several systems may be available as rem-equivalent instruments. They are rem meters and tissue equivalent proportional counters (TEPC) or some extension of these basic instruments. Two new techniques may be useful as personnel dosimeters; one, thermoluminescent detector (TLD) and the other, silicon diodes.

Needed for the Analysis

A. Design Information

1. Plant layout

2. Estimates of neutron flux at various work locations

3. Estimates of gamma and neutron spectra and dose rates at points of interest

B. R\&D Requirements

1. Radiation sources which cover the neutron and gamma spectra to be expected at a fusion reactor in the working location

2. Necessary electronic equipment to read the dosimeters

3. Measuring devices for in-situ measurements

4. Effects caused by large electromagnetic fields. 


\subsection{HIGH ENERGY PHOTONS (3-20 MeV)}

\section{Safety Concern}

Personnel protection (occupational safety)

Method of Analysis

Hethods for determining quality and quantities of these very high energy photons are required for the protection of operating personnel. Dose equivalency needs to be determined for these energies and adequate calibration techniques have to be established for both dosimeters and instrumentation.

\section{Applicable Current Technolocy}

Some attempts have been made using $\mathrm{NaI}$ detectors and $\mathrm{N}-16$ gamma rays which show some promise for useful instrumentation. Current generation personnel dosimeters may be useful if adequate calibration and interpretation procedures can be estabiished.

\section{Needed for the Analysis}

A. Design Information

Sources of very high energy photons, especially from charged particle reactions of ${ }^{6} L_{i}$, ${ }^{7} \mathrm{Li}$ and ferrous materials and their spectrum are required. This requires that construction materials and coolants have to be specified including allowable contaminants.

\section{B. R\&D Requirements}

There may be gaps in charged particle cross section data and there are no known high energy photon sources for dosimetric and instrument calibration. They will have to be developed. Dose equivalency wi 11 aiso have to be established for energies greater than $3 \mathrm{MeV}$ as well as effects of large electromagnetic fields on instruments and dosimeters. 


\subsection{OPERATIONAL AND REMOTE MAINTENANCE ANALYSIS}

These analyses are required to show that the operation and maintenance tasks specified by the designers can be carried out within estabiished guidelines for employee exposure to electromagnetic interference radiation and toxic materials present in fusion systems. The operational requirements of concern cover normal operation, maintenance during shutdown, plant accidents and during post accident return to service.

The following specific concerns indicate the types of developments that will have to be carried out.

\subsection{STRESS FACTORS FOR OPERATING PERSONNEL}

\section{Safety Concern}

Even though chemical, heat, noise, non-ionizing radiation stresses in occupational environments are recognized and dealt with in industrial situation, it will be necessary to identify what and where such stresses will be encountered in a fusion power plant so that design or other remedial measures can be considered. A case in point for the chemical concern is the possibility of production of unique alloys or compounds during operation of a full scale fusion power plant.

Method of Analysis

Methods for analyzing the nature and extent of heat, noise and chemical stresses in a fusion power plant of necessity would be based on an indepth analysis of plant systems and operations. Applicable Current Technology

An extensive literature and experience data base exists in the heat, noise and chemical stress field. Standards for protection of personnel are also generally available. Basic risks and prevention methodologies are known for these stresses, specific risks and magnitude of the problem are unknown.

Needed for the Analys is

Information needed for the analys is is related to design layout and operation of plant systems. Two main sources of current information are presently available for analysis. The fusion research laboratory experience would be of value along with conceptual reports of full scale fusion power plants.

Where presently unknown chemicals are identified some basic research may be required.

\subsection{FUSION REACTOR DECONTAMINATION AND DECOMMISSIONING}

\section{Safety Concern}

With partial decomissioning of the plasma chamber having to be carried out every 1-1/2 to 5 years and complete D\&D necessary ultimately, personnel protection or basic facility design 
information which can be identified through a $D \& D$ examination of presently conceived faulty designs should be made available to plant designers as soon as possible.

Method of Analysis

The project organization tasks and task delegations that are presently being used in the Regulatory D\&D study have been shown to be appropriate in similar cases and should be applied in the fusion facility.

Applicable Current Technology

The regulatory $D \& D$ study methodology could be directly applied.

Needed for the Analysis

The basic need of the analysis methodology recommended here is complete power ( $p l a n t$ ) layout; process component sizes, etc; and contamination or activity levels.

\subsection{WASTE HANDLING ACCIDENTS}

\section{Safety Concern}

Experience with present radioactive waste handling systems and procedures has shown that the safety of such operations can be greatly improved by the analysis of risk associated with them.

Method of Analysis

Methodology presently employed in accident analyses for SAR for environmental impact statements would be utilized.

Applicable Current Technology

Voluminous guidance on the preparation of SAR and EIS documents is available.

Needed for the Analysis

Waste handiing procedures would have to be synthesized or taken from procedures manuals for similar facilities. Unique aspects of waste management techniques may require experimenta? R\&D. Occupational and public exposure, recycling of solid radioactive waste and decontamination aspects should be addressed.

\subsection{EFFECTS OF ELECTROMAGNETIC INTERFERENCE (EMI) FIELDS}

\section{Safety Concern}

Adverse biological effects on animals have been demonstrated in lab studies.

\section{Method of Analysis}

1. Better definition of magnitude of emi fields to which workers might be exposed routinely and during accidents.

2. Long-term iaboratory studies of effects on animals of different emi field strengths bracketing the expected levels. Studies should include genetic, somatic and psychological effects. 


\section{Applicable Current Technology}

Enough laboratory experiments have been performed to indicate potential problems at high emi field strengths. Many experiments have not used adequate numbers of subjects or long enough exposure time and results are controversial. Effects detected include changes in electrical activity in the brain and heat of humans and animals, indications of changes in biological structure, genetic changes in lower organisms with possible synergism between irradiation and magnetic field exposures, exacerbation of pre-existing lesions, alteration of development of a variety of lower organisms.

Needed for the Analysis

A. Design Information

Better definition of the design of magnets and ion injectors and structural shielding and of the probability of human entry into high field zones. Definition of field leakage outside of facility, principally for evaluation of impact on birds and small mamals.

3. R\&D Requirements

Laboratory facilities including (1) equipment for generating and accurateiy measuring emi fields; (2) equipment for housing, caring for and exposing animals to field; (3) equipment for testing somatic, genetic and psychological effects on exposed and control animals. A discussion of required studies appears in "Biomagnetic Effects: A Consideration in Fusion Reactor Development," (USERDA Report BNWL-1973, Pacific Northwest Laboratories, Richiand, WA, 1976) by D. D. Mahium.

\subsection{REMOTE MAINTENANCE AND REPAIR}

\section{Safety Concern}

Personnel protection and operational safety.

\section{Method of Analysis}

Remote maintenance and repair will be essential for fusion power plants as currently conceived. Past experiences in the light water reactor (LWR) industry have shown that remote operations are costly, requiring expensive equipment, time consuming procedures and significant personnel exposure to ionizing radiation. These experiences can be expected to be part of the bases for the designs at fusion power plants.

\section{Applicable Current Technology}

The LWR fission programs have produced information about remotely operated equipment, including maintenance and repair. Another potential source of data is the nuclear rocket engine program (KIWI, ROVER). 
Needed for the Analysis

A. Design Information

Basic design data are needed including facility layout. These designs are required in advance so that estimates of radiation exposures can be performed. Those estimates will then be used to determine if remote maintenance operations can be eliminated or reduced to absolute minimum.

B. R\&D Requirements

1. Radiation source terms including neutral beam ports

2. Size and physical arrangements of components

3. Development of suitable tools, connections and other components

4. Training/practice facilities and equipment. 


\subsection{RADIOACTIVE MATERIAL INVENTORY AND LOCATION}

Establishing the inventory of radioactive materiais in a 11 components within the primary boundary of fusion systems during normal operation will be very important to the design and

safety assessment. The concentrations become source terms for assessing the effects of both primary boundary leakage and failure.

The following specific requirements are typical of the programs that will be necessary to complete these analyses.

\subsection{FIRST WALL RELEASE OF RADIOACTIVE ISOTOPES}

\section{Safety Concern}

During normal operation, production of activated products and their release (e.g., via sputtering) to the vacuum system needs to be analyzed.

During accident conditions (e.g., temperature excursion, failure of vacuum system) abnormal release of activated products, tritium held up by first radiation wall, etc., may occur.

Method of Analysis

Computer codes will be used to determine the type and amount of activated products produced. Experimental data on tritium hold-up and on contamination of the first radiation wall by activated products from the first structural wall are needed. Physical and chemical property data together with postulated normal and accident conditions will then be used to estimate the release of radioactive isotopes.

Applicable Current Technology

Computer codes and most of the cross-section data are available. At least some of the physical and chemical property data are available depending on the type of first radiation wall.

Needed for the Analysis

A. Design Information

1. Liner or coating

2. Material

3. Amount

4. Normal operating parameters

B. R\&D Requirements

1. Sputtering coefficients (both first radiation wall and first structural wall)

2. Tritium hold-up information

3. Radiation effects 


\subsection{CORROSION PRODUCT ACTIVITY CONTROL}

\section{Safety Concern}

Any liquid system, and, generally to a much lesser extent, gas system exposed all or in part to neutron radiation will develop an inventory of radioactive corrosion products. Therefore, it may be necessary to devise methods to remove corrosion products from the coolant to minimize the mobile radioactive inventory and the spread of the coolant-borne activity to piping in reactor maintenance areas. Depending on the needs and the viable approaches which develop, the cleanup of coolant activity may occur during shutdowns or while the plant is on-line. The safety incentives are a) to minimize the increase of radiation levels in areas which must be accessible to plant maintenance forces; b) to minimize radioactive inventories in equipment scheduled for disposal; c) to minimize the loss of radioactive species in case of pipe breaks in the primary coolant systems.

The principal concern is for the safety of plant personnel. However, if the primary coolant system is breached, resulting in a liquid metal fire or release of a gaseous coolant, activity losses which affect the general public will be the major concern.

Method of Analysis

The analysis will require estimates of corrosion product inventories which are likely to arise in fusion reactor systems and an assessment of the probable requirements for corrosion product removal from the primary coolants or blanket systems.

The magnitudes of potential radioactive inventories in coolant and blanket candidates will be determined for a range of fusion reactor designs, materials and operating conditions. This will be accomplished by developing computerized calculations which will permit a flexible assessment of reactor parameter effects on corrosion product inventories based on reactor designs and published corrosion data.

The calculated corrosion product inventories will provide the basis to determine the need for corrosion product removal as a function of reactor design, material, and operating conditions. The assessment also will draw on existing data from water reactor and liquid metal fast breeder reactor (LMFBR) technologies to determine the consequences of the activity and of possible methods to control it.

Applicable Current Technology

Radiation buildup in water reactors has been a long-standing concern, but methods to deal effectively with the circulating radioactive species are only partially developed. Ion exchange is an effective and relatively well jeveloped technolgy which can apply to fusion reactors using aqueous coolants in $\mathrm{Fe}-\mathrm{Ni}-\mathrm{Cr}$ and $\mathrm{Ni}-\mathrm{Cr}-\mathrm{Fe}$ systems. Techniques for filtering particulate species from circulating aqueous coolants are in the R\&D stage, but are not yet available for routine engineering applications to reactor primary systems. 
The consequences of coolant-borne activity in various water reactor configurations have been documented and are useful in assessing the safety aspects of water-cooled fusion reactors, and to some extent, systems cooled by liquid metals or molten salts.

Corrosion product removal from liquid metal reactors is in early stages of development. Some information regarding levels and distributions of activity in LMFBRs is available, providing insights to possible corrosion product behavior in fusion reactors having liquid metal coolants.

Information from the Molten Salt Breeder Reactor (MSBR) program provides some useful data regarding corrosion in molten salt systems.

Activity transport in gas-cooled fission reactors is relatively minor, consisting principally of fission product activities from leaching fuel elements. The fission products will not occur in pure fusion devices, but may be relevant to fusion-fission hybrid reactors. Tritium will provide a source of activity transport in gas-cooled fusion systems.

\section{Needed for the Analys is}

A. Design Information

1. To calculate corrosion product inventories and induced radioactivities, knowledge of the following system parameters will be required.
a. System area
b. Radiation zone area
c. Coolant and/or blanket species
d. System materials
e. Neutron fluxes and energy spectra
f. Liquid or gas flow rates
g. System temperature distributions
h. Corrosion rates of system materials

2. A calculational computer program will need to be set up to assimilate and store the above data and make corrosion product inventory calculations. Estimates of corrosion product radioactivity levels will be based on computer codes developed for water reactors or LMFBRs. Contribution of radioactive recoil atoms to the radiation inventory also will be estimated.

3. From the system design, materials and corrosion product inventory, an assessment of the probable need for a corrosion product removai system will be made, based on the safety impacts of the corrosion product activities. The assessment will include an estimate of the rate of activity buildup in the system if a removal system is not installed. The estimated levels of corrosion product activity also will provide a basis for assessing species and amounts of activity transport to the plant and environment in case of a pipe break. 
B. R\&D Requirements

1. A study is needed to analyze computerized methodology for modeling and estimating corrosion product generation and activation in water reactor and LMFBR systems. The study would provide a basis for developing methodology for similar calculations in fusion reactor systems.

2. A survey of corrosion product control and removal methods is needed to begin to define approaches which should be factored into fusion reactor designs and research and development programs.

\subsection{TRITIUM HANDLING AND STORAGE SYSTEM FAILURE AND LOSSES}

\section{Safety Concern}

Tritium handling and storage facilities must be designed to contain and transer relatively large tritium inventories involved in the plant fuel cycle. Accidental and routine tritium releases to the containment system present a potential health hazard to plant personnel. The threat to the general public is present, but can be greatly minimized if the storage system is fully enclosed in containment boundaries.

\section{Method of Analysis}

The optional approaches to tritium handling and storage in fusion systems need to be defined and assessed for strengths and weaknesses from the standpoint of safety. Much useful information can be derived from systematic assessments of practices and experience in laboratories which presently handle large tritium inventories. In some areas, additional research and development will be required to expand current tritium handling technology.

\section{Appi icable Current Technology}

Several U.S. laboratories have handled large tritium inventories over the past two decades. Much tritium handling information, useful to fusion technology, has been documented. Additional useful information can be derived by systematic assessment of tritium handling and loss data. For example, tritium losses associated with major and minor maintenance can be assessed and documented more completely than is now available.

The details of several major accidental tritium releases have been documented, including causes and environmental consequences. The fusion safety program would benefit from an expanded summary of both documented, and where possible, undocumented case histories of tritium system failures. The principal thrust of this analysis would be to identify the reiative importance of operator error and component failure in contributing to accidental releases. The study would provide a basis to identify potential design aspects and materials selection which would minimize the likelihood of accidental and routine releases in fusion systems. 


\section{Needed for the Anaiysis}

A. Design Information

1. Details regarding fusion plant tritium handling system layout and major components.

2. Definition of systen construction options which are viable (e.g., welded versus flanged piping).

3. Definition of the numbers, sizes, and types of valves, flanges (if any), and pipes required.

4. Definition of the probable tritium inventories and transfer requirements in each part of the tritium handing and storage system.

5. Definition of the options for tritium storage (e.g., gaseous, liquid or solid), their strengths and weaknesses, and their operating conditions (e.g., temperatures and tritium pressures).

6. Definition of tritium containment boundary requirements and design options.

7. Definition of cover gas cleanup requirements and options.

8. Definition of the types and frequencies of major and minor maintenance to equipment which is exposed to tritium.

9. Better definitions of the tritium loss pathways which have the most serious environmental consequences.

B. R\&D Requirements

1. Better tritium permeation data in potential fusion reactor operating regimes, particularly at tritium pressures less than one torr. Investigation of corrosion as a mechanism for tritium permeation.

2. Better definition of tritium 1055 rates from components such as flanges and valves, as a function of design, age, material, etc.

3. Better definition of tritium storage options where large tritium inventories are involved. For example, metal tritides are effective for storing small tritium inventories. What are the prospects and drawbacks for scaling them to large inventories?

4. Eetter definition of potential tritium losses during major and minor maintenance.

5. Better definition of the kinetics of tritium reaction and exchange with gases, liquids and solids which are likely to occur in fusion reactors.

6. Development of tritium barrier materials. 


\subsection{DISPERSION}

Development of a Source Term - Within the analyses for containment, the amount and form of hazardous material available for release must be developed. Involved are the various retention factors that realistically assess the quantities being held up within the vessel. Also included are the physical and sometimes chemical form of the pollutant as well as physical conditions at the point of release.

The quantity available for release becomes the source term. As indicated below, eventually this analys is must be developed for fusion reactors.

\subsection{PUBLIC IMPACT ESTIMATES}

Models - Many models for dispersion of pollutants and for impact on the public have been developed for safety analyses for fission reactors. The pollutants considered have been radioactive. The development of models to describe the dispersal and public impact of all hazardous materials can be expected to be developed.

of the several models available, the computer programs SUBDOSA and DACRIN are used here to calculate the dose to humans following accidental atmospheric releases of radionuclides. SUBDOSA calculates the dose from airborne radionuclides external to the body. DACRIN is used to calculate the dose resulting from radionuclides deposited in the body and its organs via inhalation. DACRIN uses the ICRP Task Group Lung Model (TGLM) to calculate the uptake of radioactive materials into the body and the resultant dose to various body organs. Details of these computer codes and the modeling techniques used are contained in references 30 and 31 of the text.

These programs calculate doses as a function of: (1) quantity, physical form and type of material release, (2) duration of release, (3) atmospheric conditions during release, (4) horizontal distance from release point, and (5) release height. 


\subsection{LIQUID METAL REACTIONS}

In accident scenarios which result in failure of the primary boundary, lithium will be released to the secondary containment. Liquid metal reaction with both air and/or concrete could contribute to the energy released in such an accident. The increased energy release could contribute to containment overpressurization or to boiling off more activation products than would normally be expected for a given accident.

Specific programs are not identified here, but they would relate very closely to some R\&D tasks now in progress in the LMFBR program. The programs would have to be expanded to include studies with lithium.

\section{REFERENCES}

1. E. Kasahara, et a1., "Studies on Plasma Jets," JSME Builetin, Vol. 7, pp. 590-596, 1964.

2. J. F. Skrivan, et al., "Heat Transfer from Plasmas to Waxes-Cooled Tubes," I\&EC Process Design and Develooment, Vol. 4, pp. 371-379, 1965. 
A. A. Churm ERDA Chicago Patent Group

$9800 \mathrm{~S}$. Cass Avenue

Argonne, IL 60439

J. W. Beal

ERDA Div. of Magnetic

Fusion Energy

Washington, DC 20545

S. O. Dean

ERDA Div. of Magnetic

Fusion Energy

Washington, DC 20545

E. E. Kintner

ERDA Div. of Magnetic

Fusion Energy

Washington, DC 20545

J. M. Williams

ERDA Div. of Magnetic

Fusion Energy

Washington, DC 20545

J. N. Grace

ERDA Div. of Magnetic

Fusion Energy

Washington, DC 20545

J. Baublitz

ERDA Div. of Magnetic

Fusion Energy

Washington, DC 20545

3 F. E. Coffman

ERDA Div. of Magnetic

Fusion Energy

Washington, DC 20545

J. F. Decker

ERDA Div. of Magnetic

Fusion Energy

Washington, DC 20545

3 K. M. Zwilsky

ERDA Div. of Magnetic

Fusion Energy

Washington, DC 20545

Dr. Philip M. Stone

ERDA Applied Plasma Physics

Program

Washington, DC 20545

G. W. Kuswa

ERDA Div. of Laser Fusion

Washington, DC 20545
OFFSITE

R. Blaunstein

ERDA Div. of Biomedical

and Environmental Research

Washington, DC 20545

H. M. Busey

ERDA Div. of Military

Application

Washington, DC 20545

M. A. Bell

ERDA Div. of Safety

Standards and Compliance

washington, DC 20545

27 ERDA Technical Information

Center

M. S. Kaminsky

Argonne Nationa? Laboratory

9700 S. Cass Avenue

Argonne, IL 60439

V. A. Maroni

Argonne National Laboratory

9700 S. Cass Avenue

Argonne, IL 60439

P. M. Persiani

Argonne National Laboratory

9700 S. Cass Avenue

Argonne, IL 60439

M. Petrick

Engineering and Technology

Division

Argonne National Laboratory

9700 S. Cass Avenue

Argonne, IL 60439

W. E. Parkins, Manager

Atomics International

Component Engineering and

Technology Division

North American Rockwell

P. O. Box 309

Canoga Park, CA 91304

D. Gurinsky

Brookhaven National Laboratory ERDA Brookhaven Area Office Upton, NY 11973

H. J. Kouts

Brookhaven National Laboratory ERDA Brookhaven Area Office Upton, NY 11973

5. Pearlstein

Brookhaven National Laboratory

ERDA Brookhaven Area Office

Upton, NY 11973
OFFSITE

J. R. Powell

Brookhaven National Laboratory ERDA Brookhaven Area Office Upton, NY 11973

A. J. Impink, Jr. Carnegie Melion University

Pittsburgh, PA 15213

R. A. Gross

Plasma Research Laboratory

236 SW Mudd Bldg.

Columbia University

New York, NY 10027

W. C. Gough

Electric Power Research Inst.

3412 Hillview Ave.

Palo Alto, CA 94304

G. R. Hopkins

Gulf General Atomic

P.0. Box 1111

San Diego, CA 92112

Zeinab Sabri

Iowa State University

261 Sweeney Hall

Nuclear Engineering Department

Ames, IA 50010

R. Borg

Lawrence Livermore Laboratory

P.0. Box 808

Livermore, CA 94550

T. K. Fowler

Lawrence Livermore Laboratory

P.0. Box 808

Livermore, CA 94550

R. Moir

Lawrence Livermore Laboratory

P.0. Box 808

Livermore, CA 94550

A. Carl Haussmann

Lawrence Livermore Laboratory

P.0. Box 808

Livermore, CA 94550

J. Hovingh

Lawrence Livermore Laboratory

P.0. Box 808

Livermore, CA 94550

R. F. Post

Lawrence Livermore Laboratory

P.0. Box 808

Livermore, CA 94550

C. J. Taylor

Lawrence Livermore Laboratory

P.0. Box 808

Livermore, CA 94550 
R. Werner

Lawrence Livermore

Laboratory

P.0. Box 808

Livermore, CA 94550

L. L. Wood

Lawrence Livermore

Laboratory

P.0. Box 808

Livermore, CA 94550

W. Baver

Division Supervisor

of Physical Research

Sandia Labs Livermore

Livermore, CA 94550

L. Booth

Los Alamos Scientific

Laboratory

CTN Research

P.0. Box 1663

Los Alamos, NM 87544

0. J. Dudziak

Los Alamos Scientific

Laboratory

CTN Research

P.0. Box 1663

Los Alamos, NM 87544

D. B. Henderson

Los Alamos Scientific

Laboratory

CTN Research

P.0. Box 1663

Los Alamos, NM 87544

E. L. Kemp

Los Alamos Scientific

Laboratory

CTN Research

P.0. Box 1663

Los Alamos, NM 87544

F. L. Ride

Los Alamos Scientific

Laboratory

CTN Research

P.0. Box 1663

Los Alamos, NM 87544

L. Stewart

Los Alamos Scientific

Lacoratory

CTN Research

P.0. Box 1663

Los Alamos, NM 87544

K. Thomassen

Los Alamos Scientific

Laboratory

CTN Research

P.0. Box 1663

Los Alamos, NM 87544
0. K. Harling

Massachusetts institute of

Technology

Cambridge, MA 02139

Bruno Coppi

Department of Physics

Massachusetts Institute of

Technology

Cambridge, MA 02139

L. Lidsky

Dept. of Nuclear Engineering

Massachusetts Institute of

Technology

Cambridge, MA 02139

Norm Rasmussen

Dept. of Nuclear Engineering

Massachusetts Institute of

Technology

Cambridge, MA 02139

David Rose

Massachusetts Institute of

Technology

Cambridge, MA 02139

R. E. Stickney

Mechanical Engineering

Massachusetts Institute of

Technology

Cambridge, MA 02139

J. J. Reinmann

NASA - Lewis Research Center

2100 Bookpark Rd.

Cleveland, OH 44135

Vincent Arp

National Bureau of Standards

Cryogenics Division

Boulder, CO 80302

J. F. Clarke

Oak Ridge National

Laboratory

P.O. Box Y

Oak Ridge, TN 37830

A. P. Fraas

Oak Ridge Nationa?

Laboratory

P.O. Box Y

oak Ridge, TN 37830

J. Rand McNally, Jr. Oak Ridge National

Laboratory

P. 0. Box $Y$

Oak Ridge, TN 37830

D. Steiner

Oak Ridge National

Laboratory

P.O. Box Y

Oak Ridge, TN 37830
J. Scott

Oak Ridge National Laboratory

P. 0. Box $X$

Oak Ridge, TN 37830

J. Banford

Physics International

2700 Merced St.

San Leandro, CA 94577

R. A. Huse

Public Service Electric

and Gas Co.

80 Park Place

Newark, NJ 07101

M. Gottlieb

Princeton University, PPPL

P. 0. Box 451

Princeton, NJ 08540

R. G. Mills

Princeton University

P.0. Box $45 i$

Princeton, NJ 08540

E. C. Tanner

Princeton University

P.0. 80x 451

Princeton, NJ 08540

H. Perkins

Dept. of Chemistry

Princeton University

Princeton, NJ 06540

R. E. Gold

303 Sayre Hall

Forrestal Campus

P.0. Box 451

Princeton, NJ 06540

M. Kristiansen

Texas Tech. University

Lubbock, TX 79409

A. F. Haught

United Aircraft Research Lab.

United Aircraft Corporation

East Hartford, CT 06108

L. Levine

U.S. Naval Research Laboratory Washington, DC 20390

C. 2. Serpan, Jr.

U.S. Naval Research Laboratory Washington, DC 20390

Francis Chen

University of California

Electronics Research

Laboratory

College of Engineering

Berkeley, CA 94720 
A. J. Lichtenberg University of California Electronics Research Laboratory

College of Engineering

Berkeley, CA 94720

Dave Okrent

U.C.L.A.

Los Angeles, CA 90024

C. D. Hendricks University of Illinois Nuclear Engineering

Laboratory

Urbana, IL 61801

G. H. Miley University of Illinois

Nuclear Engineering Laboratory

Urbana, IL 61801

Terry Kammash University of Michigan Nuclear Engineering Department

Ann Arbor, MI 48105

Dean Abrahamson University of Minnesota School of Public Affairs

Social Science

Building/309

Minneapolis, MN 55455

W. G. Davey

University of Texas

Department of Physics

Austin, TX 78712

E. Linn Draper, Jr. University of Texas Department of Physics Austin, TX 78712

W. E. Drummond University of Texas Department of Physics Austin, TX 78712

Abraham Hertzberg University of Washington Aerospace Research Laboratory

316 Guggenheim Seattle, WA 98105

A. L. Babb University of Washington Nuclear Engineering Department

- Seattle, WA 98105
R. Conn University of Wisconsin Nuclear Engineering

Department

Madison, WI 53706

G. L. Kulcinski

University of Wisconsin

Nuclear Eng ineering

Department

Madison, WI 53706

C. W. Maynard

University of Wisconsin

Nuclear Engineering

Department

Madison, WI 53706

D. Lichtman

Department of Physics

University of Wisconsin

Milwaukee, WE 53201

E. E. Donaldson

Washington State University

Deparment of Physics

Pullman, WA 99163

D. D. Mahlum

Division of Biomedical

and Environmental Research

Washington, OC 20545

J. V. Vanston

Engineering Science

Building

University of Texas

Austin, TX 78712

Leslie S. Ramsey

450 North 5 th Street

Indiana, PA 15701 
ERDA Richland Operations Office

W. A. Burns

Atlantic Richfield Hanford Company

J. D. Kaser

Hanford Engineering

Development Labs

D. G. Doran

H. H. Yoshikawa

\section{Battelle-Northwest}

D. T. Aase

G. S. Allison

T. W. Ambrose

D. G. Atteridge

D. A. Baker

J. L. Bates

M. A. Bayne

E. R. Bradley

J. L. Brimhall

R. L. Brodzinski

R. J. Brouns

L. R. Bunnell

L. L. Burger

S. H. Bush

N. E. Carter

T. D. Chikalia

10 R. G. Clark

T. L. Criswell

S. D. Dahlgren

M. T. Dana

D. E. Deonigi

R. L. Dillon

$D$. A. Dingee

P. J. Dionne

B. H. Duane

J. W. Finnigan

J. C. Fox

J. J. Fuquay

J. E. Garnier

R. D. Gasti

B. F. Gore

J. N. Hartley

A. J. Haverfield

U. P. Jenquin

A. B. Johnson, Jr.

R. H. Jones

T. J. Kabele

W. S. Kelly

H. E. Kissinger

D. A. Kottwitz

N. Laegried

B. R. Leonard, ur

D. L. Lessor

H. 3. Liemohn

R. C. Li ikala

M. A. Mckinnon

R. F. Maness

$R$. P. ilarshall
Battelle-Northwest - Continued

E. S. Murphy

$R$. D. ivelson

D. F. Newman

R. E. Nightingale

D. E. Olesen

L. T. Pedersen

R. T. Perry

D. R. Pratt

L. A. Rancitelli

J. F. Remark

U. S. Renné

R. E. Rhoads

W. O. Richmond

W. F. Sandusky

L. C. Schmid

iv. M. Sherer

E. P. Simonen

R. 1. Smith

J. K. Soldat

C. W. Stewart

K. B. Stewart

R. W. Stewart

J. A. Strand

D. L. Styris

A. M. Sutey

V. L. Teofilo

G. L. Tingey

M. T. Thomas

R. C. Thompson

L. H. Toburen

T. J. Trapp

R. Wang

R. E. Westerman

L. D. Williams

10 J. R. Young

M. G. Zimmerman

1 Technical Publications (BH)

5 Technical Information 